Portland State University

PDXScholar

5-3-1971

\title{
Differences in the Attitudes of Church-Attending Catholics Toward Changes in Religious Beliefs and Practices Correlated with Age and Education
}

Cletus Michael Kirkpatrick

Portland State University

Follow this and additional works at: https://pdxscholar.library.pdx.edu/open_access_etds

Part of the Christian Denominations and Sects Commons, Comparative Methodologies and Theories Commons, and the Religious Thought, Theology and Philosophy of Religion Commons Let us know how access to this document benefits you.

\section{Recommended Citation}

Kirkpatrick, Cletus Michael, "Differences in the Attitudes of Church-Attending Catholics Toward Changes in Religious Beliefs and Practices Correlated with Age and Education" (1971). Dissertations and Theses. Paper 1428.

https://doi.org/10.15760/etd.1427

This Thesis is brought to you for free and open access. It has been accepted for inclusion in Dissertations and Theses by an authorized administrator of PDXScholar. Please contact us if we can make this document more accessible: pdxscholar@pdx.edu. 
AN ABSTRACT of THE THESIS of Cletus Michael Kirkpatrick for the Master of Science in Psychology presented May 3, 1971.

Title: Differences in the Attitudes of Church-attending Catholics toward Changes in Religious Beliefs and Practices Correlated with Age and Education.

APPROVED BY MEMBERS OF THE THESIS COMMITTEE:
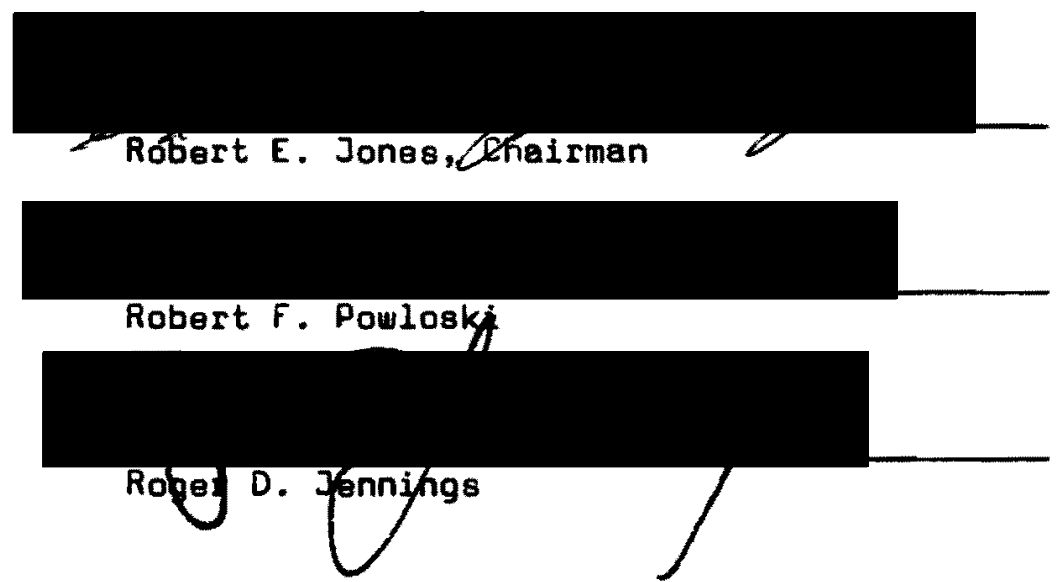

A field survay was conducted to investigate differences in attitudes toward religious changes in a population of church-going Romen Catholics. An attitude scale comprising twenty items referring to changes in the Catholic Church was prepared during pilot studies. Half of the items referred to changes already occurring, and half to proposed or possible future changes. The attitude scale was administered to a sample of parishes in the Roman Catholic diocese of Baker, Oregon. It was administered during regular Sunday services to take advantage of the seliency effect of group membership.

A response to an item of the scale indicating the subject's agreement with the change was operationally defined as a liberal 
response. A response indicating disagresment with change was considered a conservative response. It was hypothesized that churchgoing Catholics would be more liberal or accepting of changes already instituted in the Church than they would be of merely possible changes. The results supported this hypothesis.

Total scores on the scale were correlated with the age and education of the respondents. The results support the hypothesis that attitudes toward religious changes covary with age and education much like other social attitudes, es the younger and more educated respondents showed more liberalism or acceptance of change than did older and less educated respondents.

A detailed analysis of several items shows a wide divergence between parishoners' attitudes and ecclesiastical dogma. The results of the research also indicate that in the population survayed there was a great deal of intragroup variation in attitudes toward religi ous changes. 
Difrenences in the atrituoes of chinch-atrewains

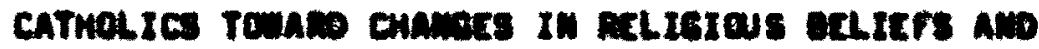

macticcs conmelated urm act and coucarrem

by

CLrTus michacl ximentaicx

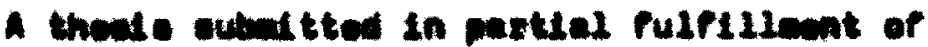
the roqulsmente for the degnee of

enstre or scicuce

in

osrewerey

Dartaland State Univeretty

1971 
To the errice of subunte strotest

The mume of the Comattes eppreve the thesls of

Clotue Mlehael KIrkpotzlek presented eny 3, 1571.

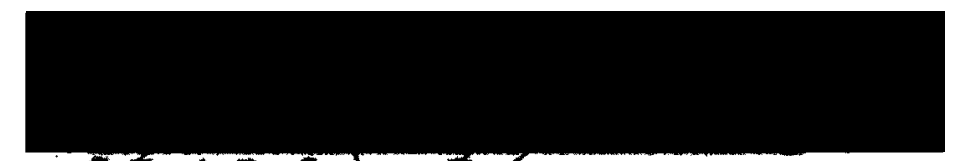

Assort E. Jenwe, Gorirman
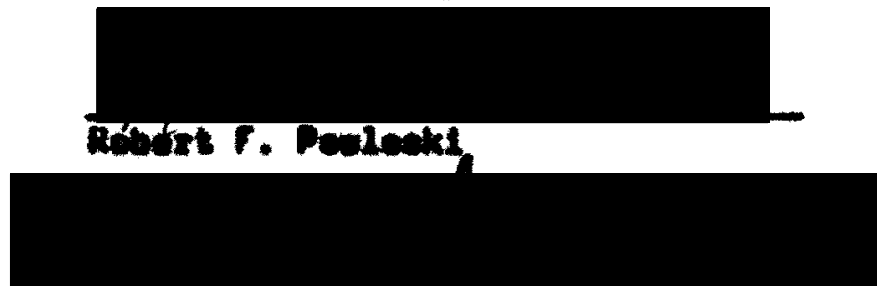

andey.
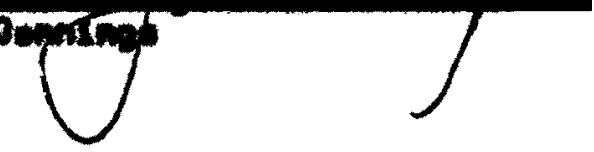

Apances:

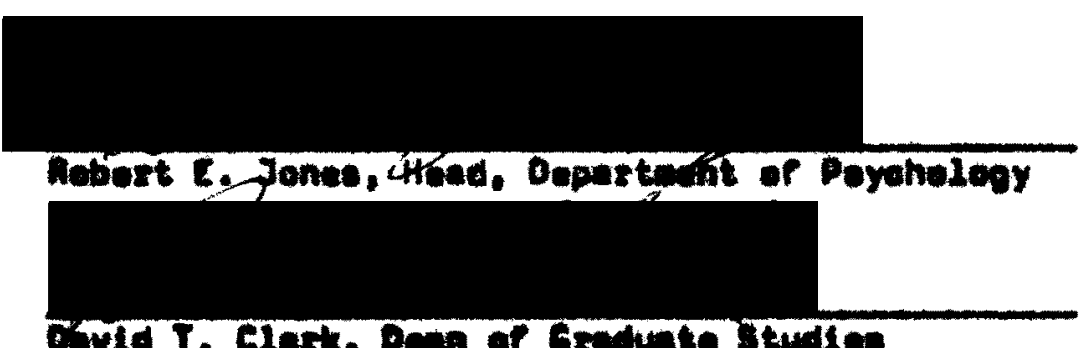

Esvid T. Clack, Dasa of Gewluate Studieo

aey 3, 1972 
Am:inimine

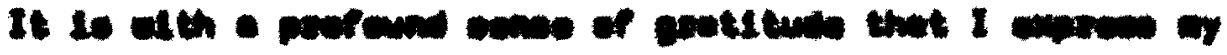

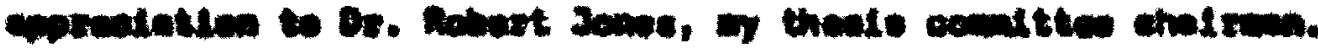

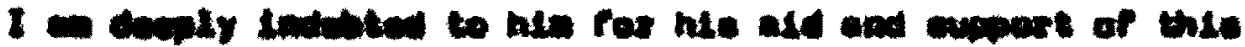

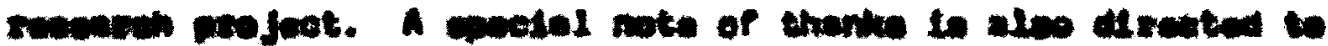

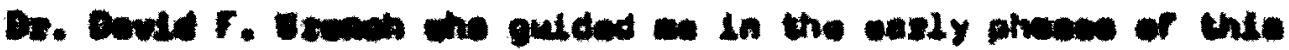

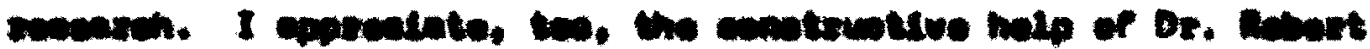

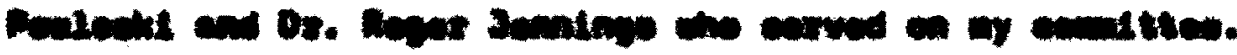

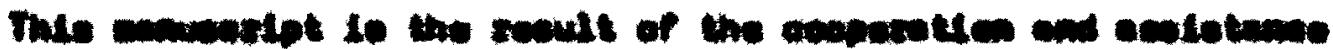

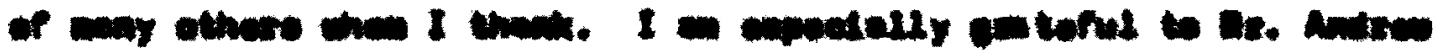

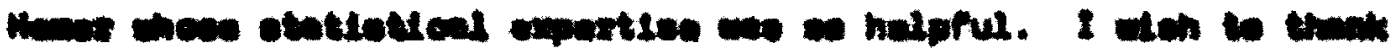

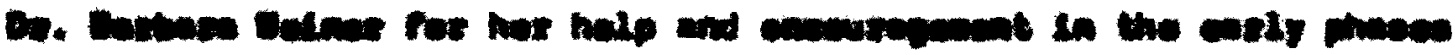

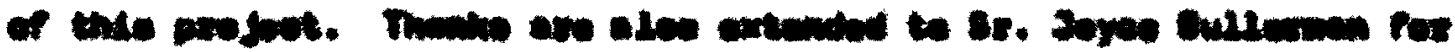

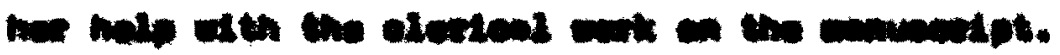


TAQLE of CONTEHTS

PACE

Acknoledenents $\quad . . . . . . . . . . . . . . . . .111$

LIST of TALES $\quad . . . . . . . . . . . . . . . . \quad$ vi

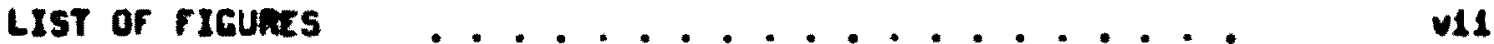

QHapten

1 Intnoouction ,.................. 1

Rellgleus Attltudes and Generel Ateltude hesenrch ............. 5

Socled and Rellglaus Attltudes ........ 6

Age and Educetion in Soclal and

Relselous Attitudes ........... 9

Comerel Characterietice of the Precent study ............................ 12

Sallenoy ....................... 15

II PROCEDURE ....................... 17

Eathods - Part I.............. 17

Sonde Conotruction

Final Field Teet

Wothode - Pert II ..............

subjecte

Emperimentex

Age and tuostional Lovele

Prodictiono $\quad . . . . . . . . . .$. 
CHAPTER

II RESULTS

Goneral Resulte

Some Individual Itome . . . . . . . . . .

IV DISCUSSION AMD IDPLICATIONS

Generel Diecuesion . . . . . . . . . .

Dlacuseion of Some Indluldual Iteas .....

furthar Raserch Posdbllties .......

REFEAENCES

APPEDDIX
PAge

26

26

31

38

38

41

47

49

52 


\section{LIST OF TABLES}

\section{TABLE}

PAGE

I Number of Reepondents in Each Age and Educationel

Group and Group Mean on Attitude Scal. ..... 


\section{LIST of FIGUAES}

\section{froune}

1. Ican soores on the ettstude coede of the four cheotionil lovele pletted egeinet epe. The higher the coeres on the eode, the ware 11 boral or acoopting of ohenge are the respendonte......

2. Eoen coeren on the attstude eade of the three ope groupe plettod egalnot eduostlomel love. The highar the soeree on the coule, the core liberel or eccepting of change are the reapendents......

3. The percontege in ench group egreelng with ltem 6. Item 61 Diwerod-rcoarrled Cotholles should be pormitted to recelve the enermente (eepoeldily Comunion) oven though thats ecoond nerriege is Invalld according to normal standardo... . . . .

4. The porcentege in each group diecgrealng with 1tem 11. Item 11: Prleste phould mot be alleoed

5. The porrentege in caoh group diegroolng oith 1tem 13. Ite 131 Jemon mut nover be allowed to becoens prleots................

6. The porcentege in each group diecgrealm with 1tem 16. Iten 16t To be a ped Catholic it is neceseery to zofrein frem any we of extificiel birth control devices and the birth eontrol p11. 


\section{cingran 1}

\section{InTramet ro:}

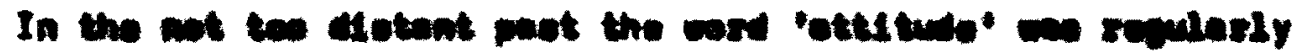

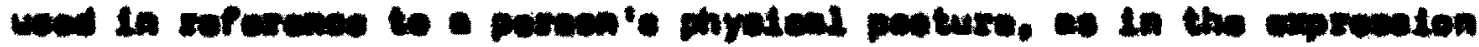

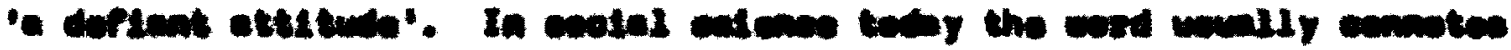

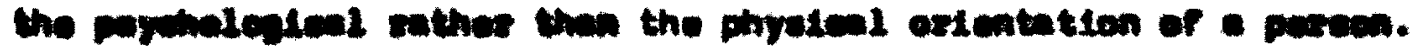

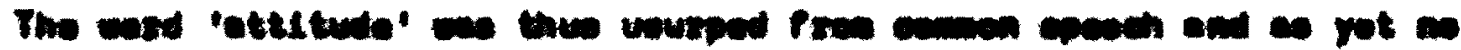

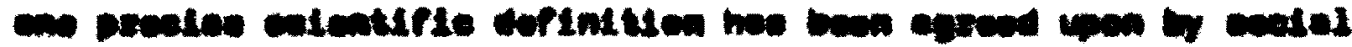
calentets.

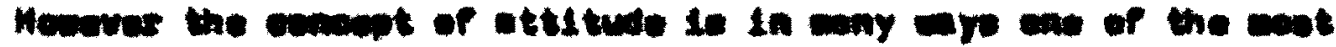

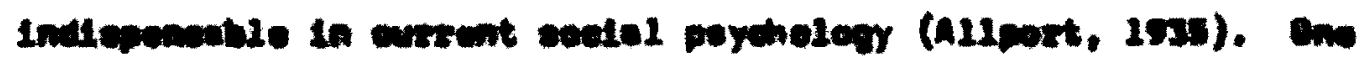

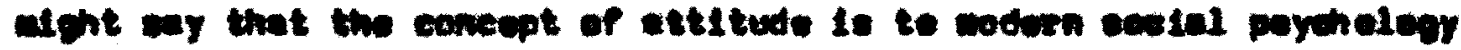

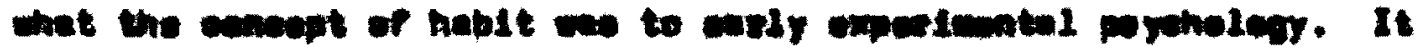

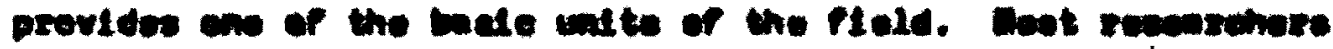

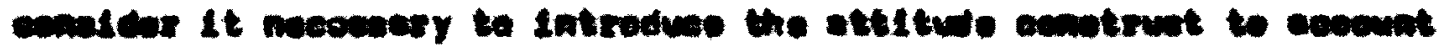

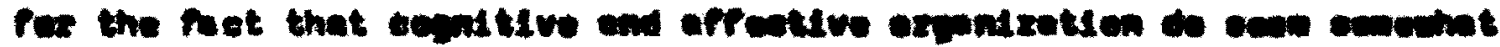

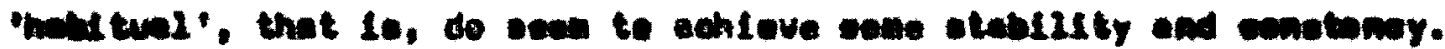

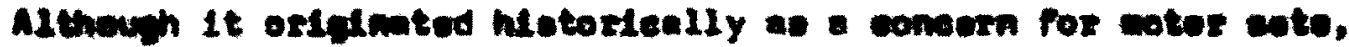

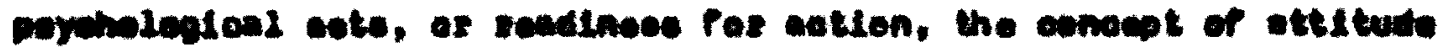

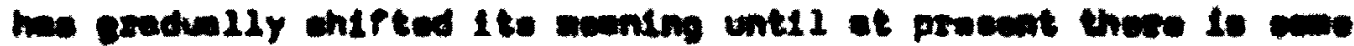

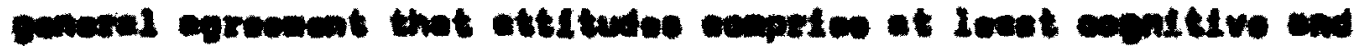

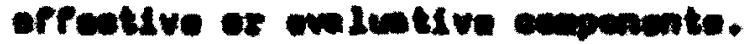




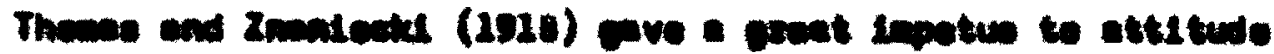

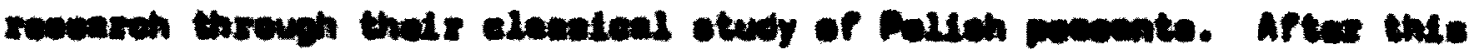

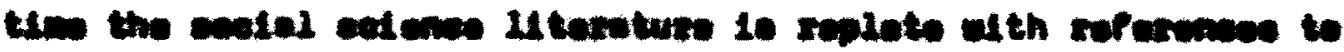
otudice of ettetudes.

The volum of otudies ho left cooted ealeme it th may

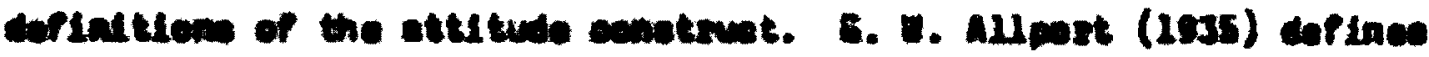

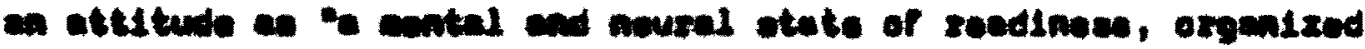

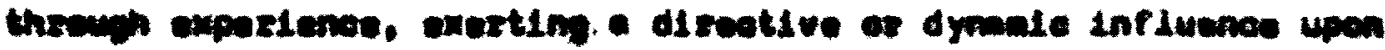

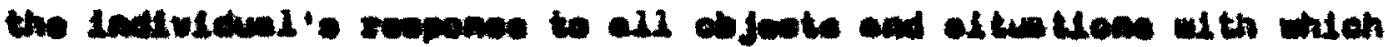

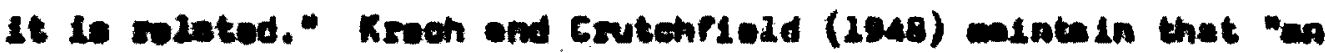

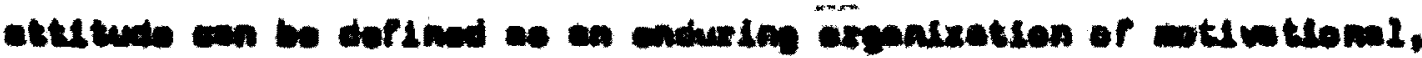

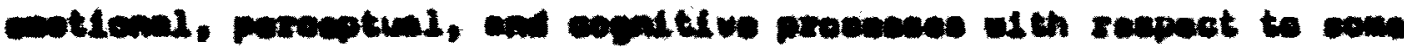

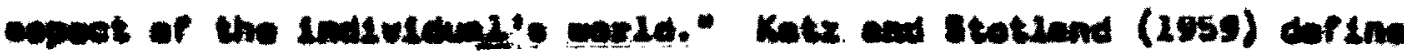

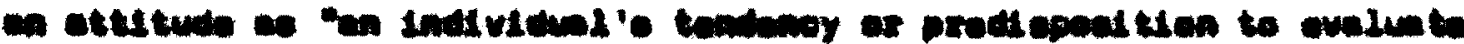

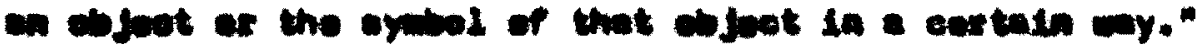

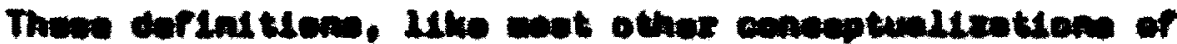

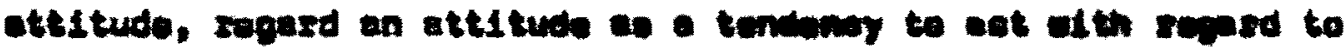

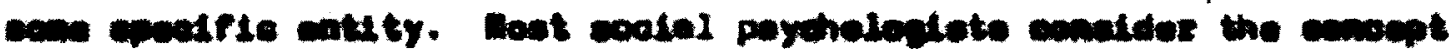

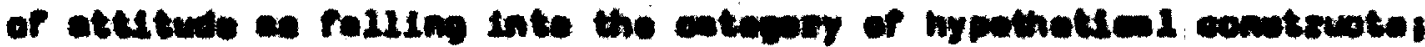

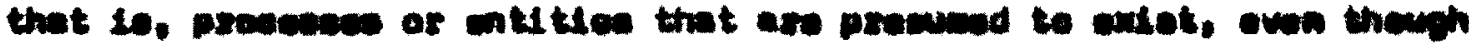

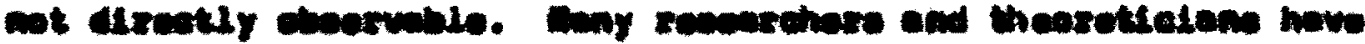

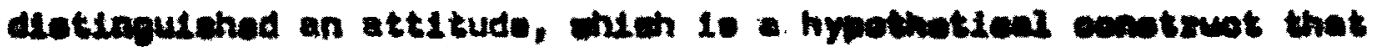

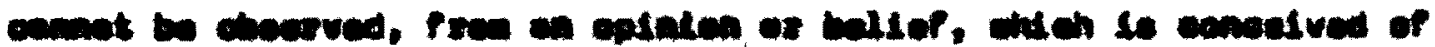

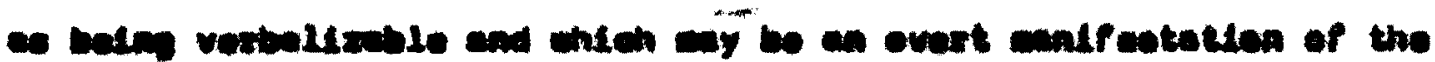

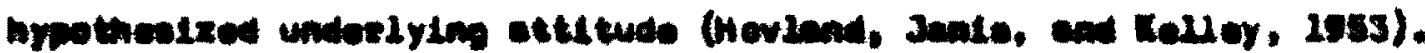

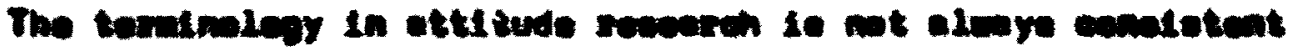

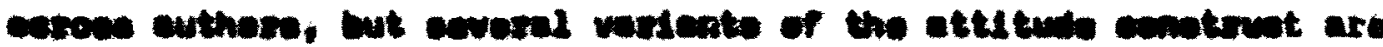




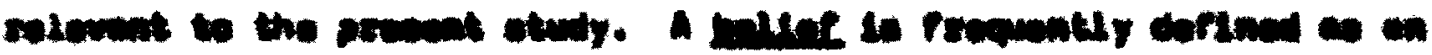

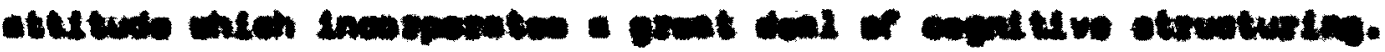

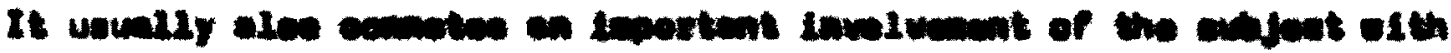

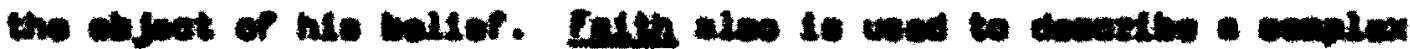

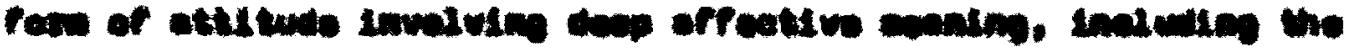

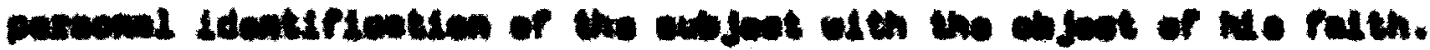

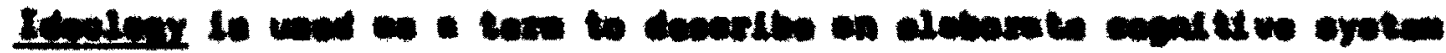

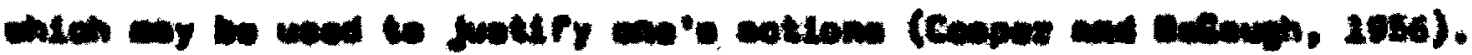

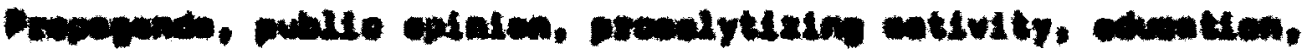

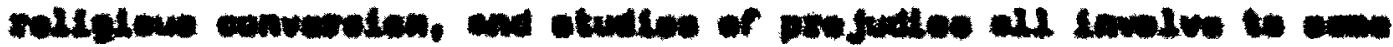

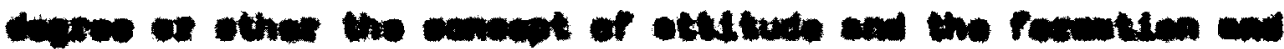

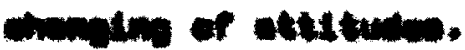

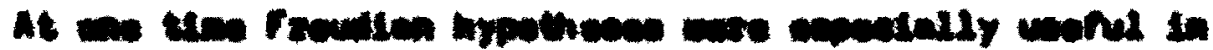

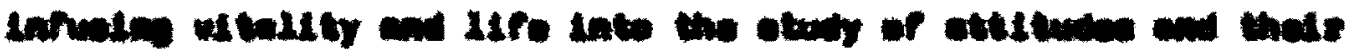

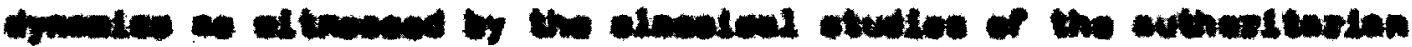

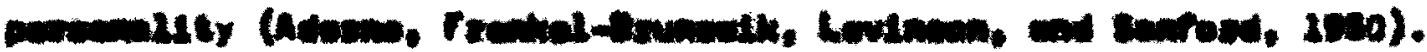

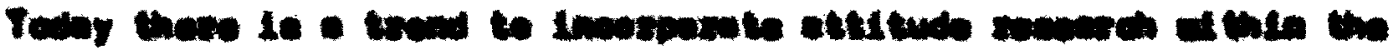

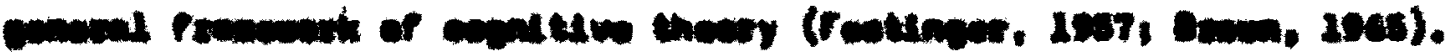

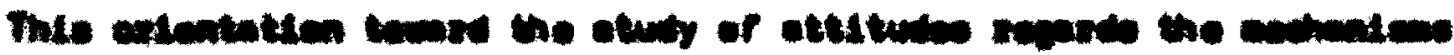

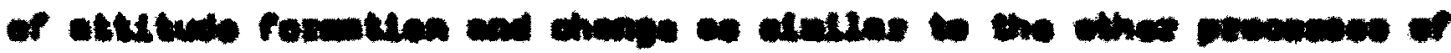

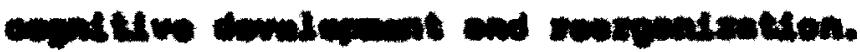

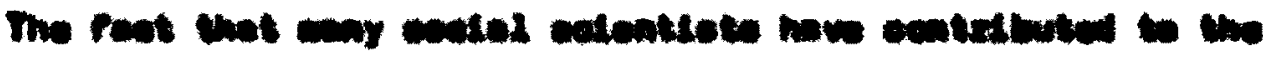

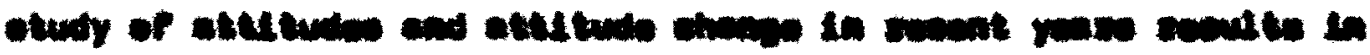

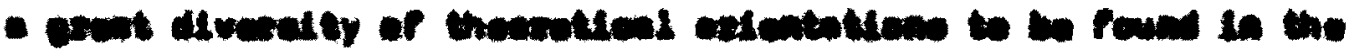

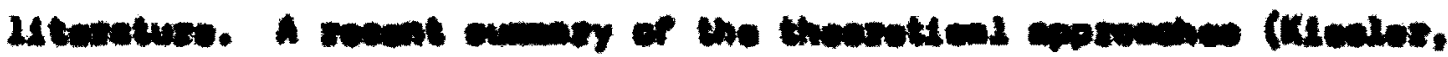

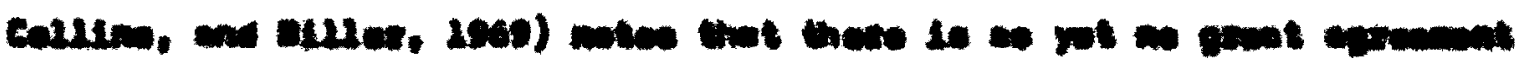

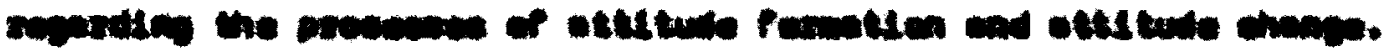




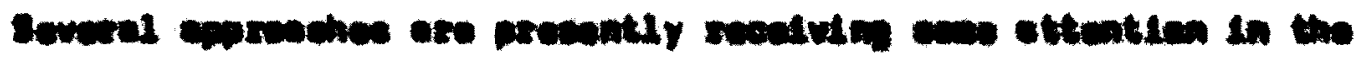

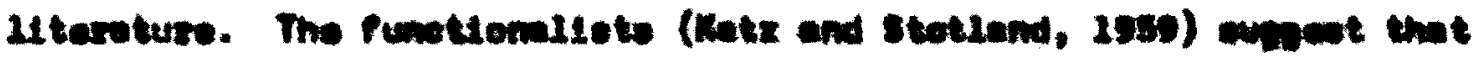

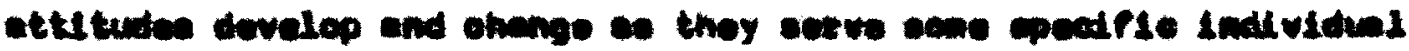

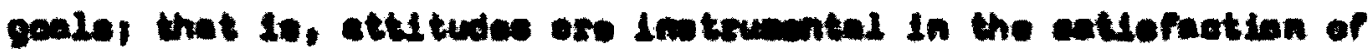

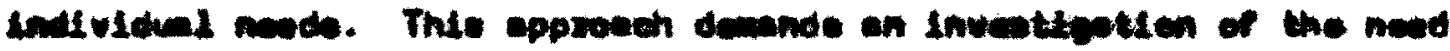

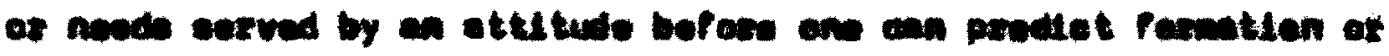
chane of attstudas.

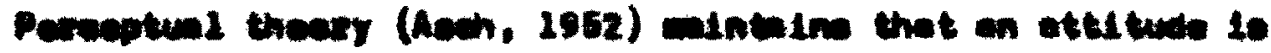

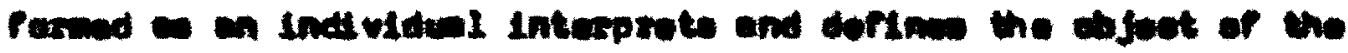
attitude. The attitude then change as the Individual mincorpoto or sudefine the attitude ebjet.

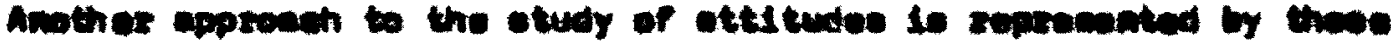

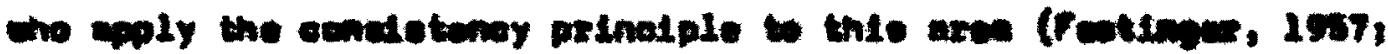

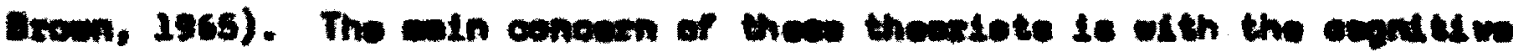

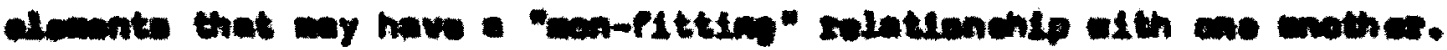

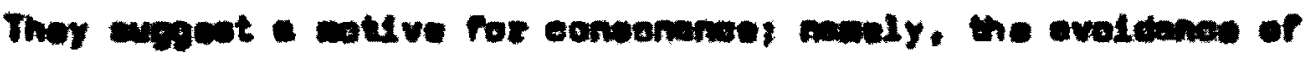

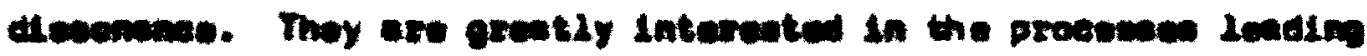

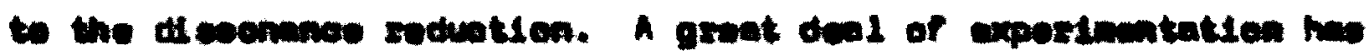

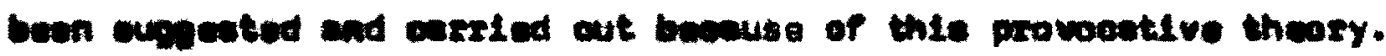

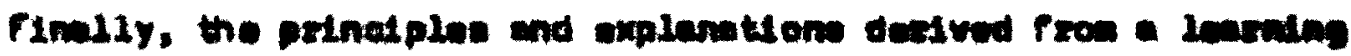

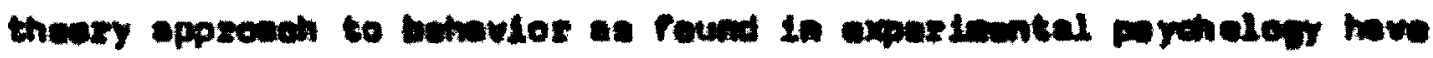

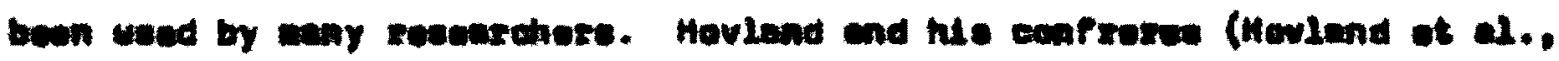

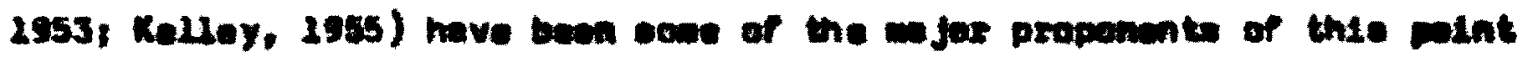
of viw. It is diffioult to find conoled ethtement of the bane

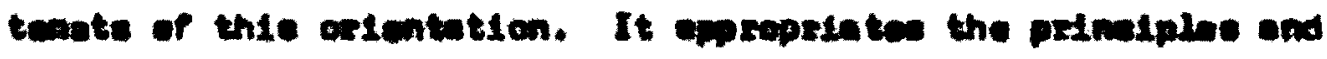

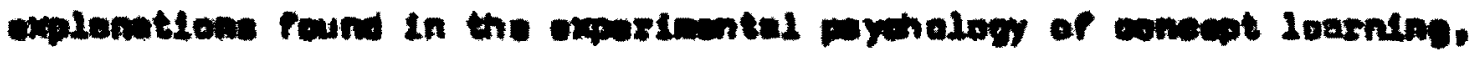




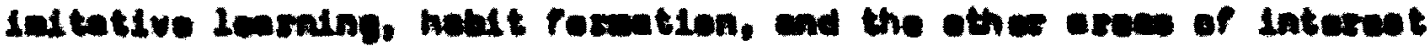
to Iovering theodote.

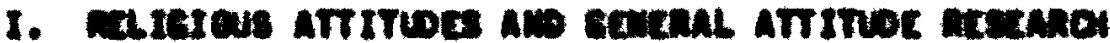

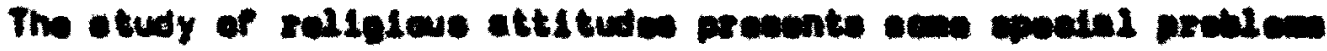

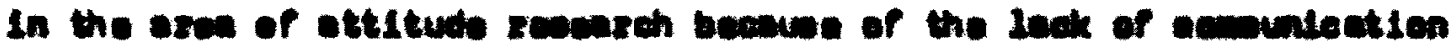

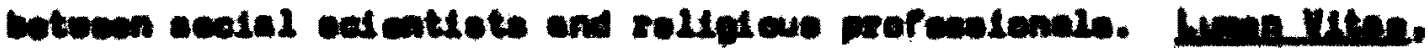

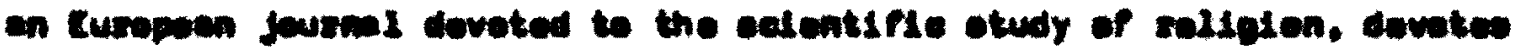

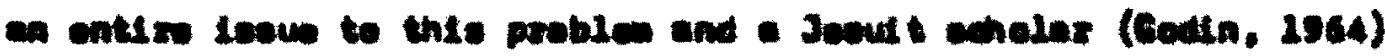

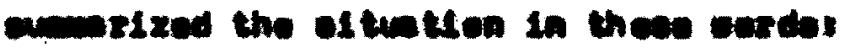

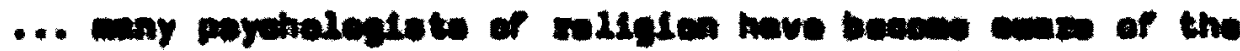

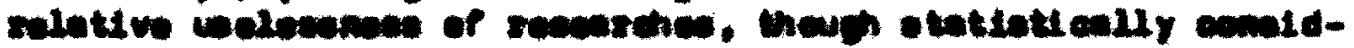

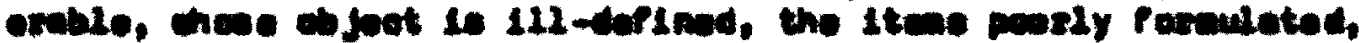

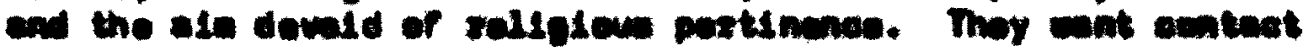

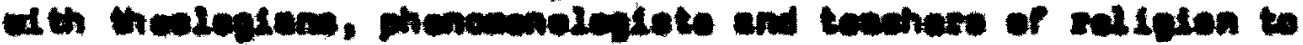

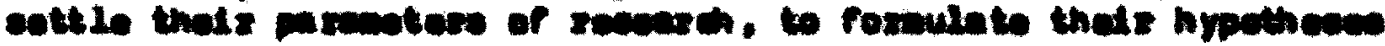

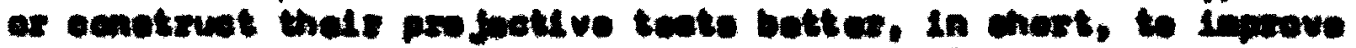

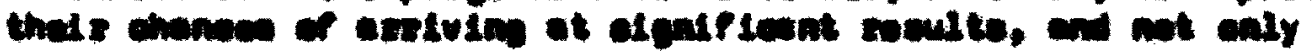

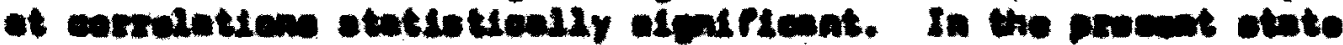

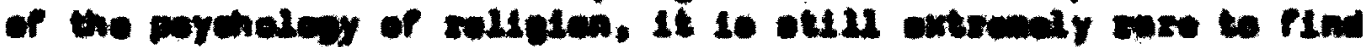

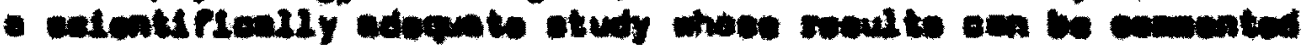

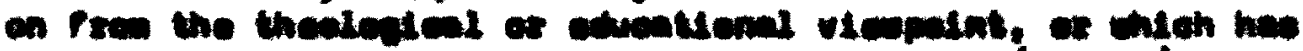

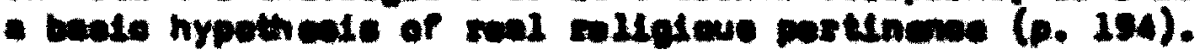

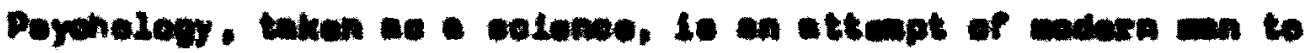

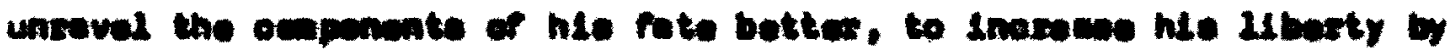

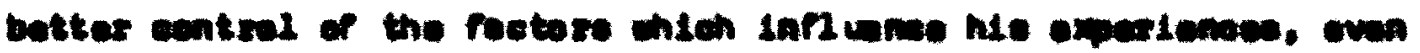

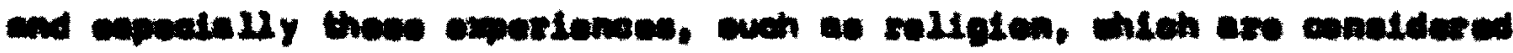

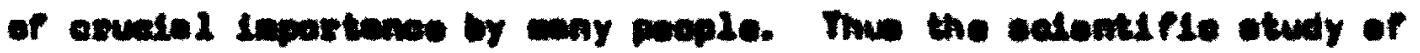

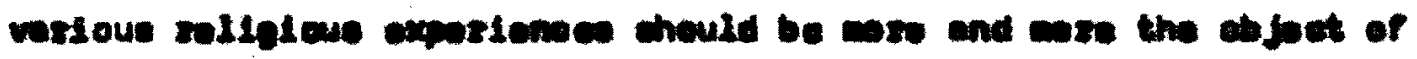

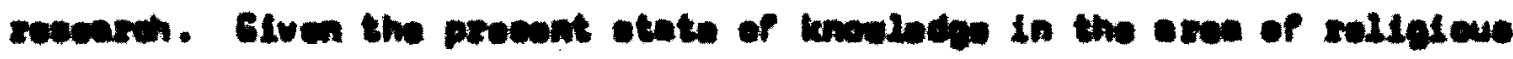

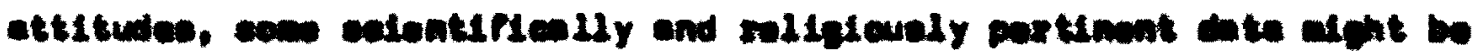
on ald in theory amotruation and an ladieter of relovent valobles. 


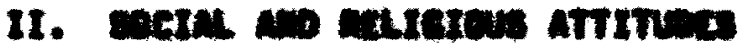

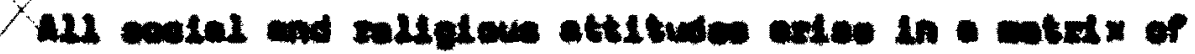

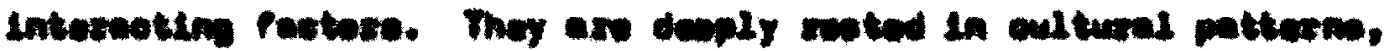

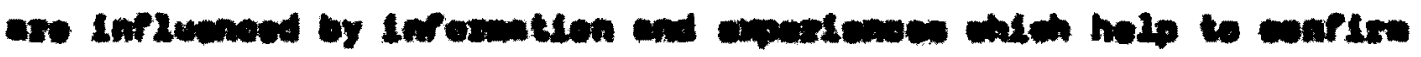

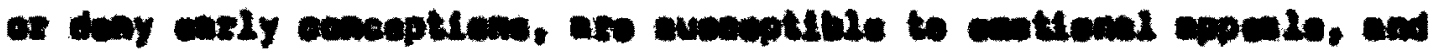

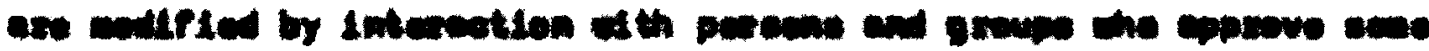

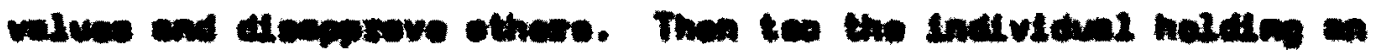

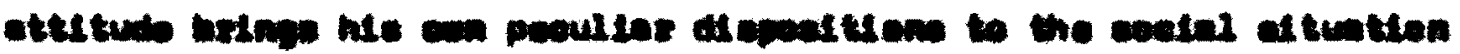

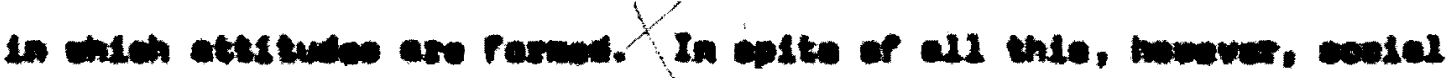

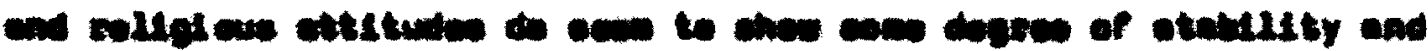

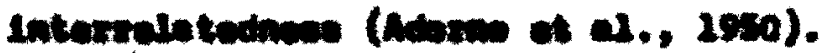

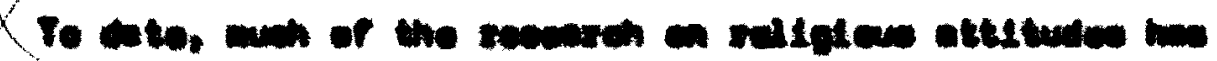

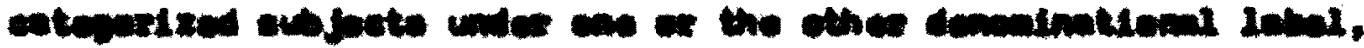

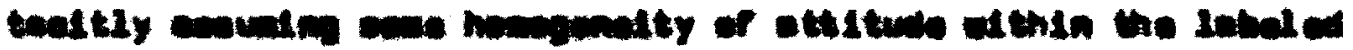

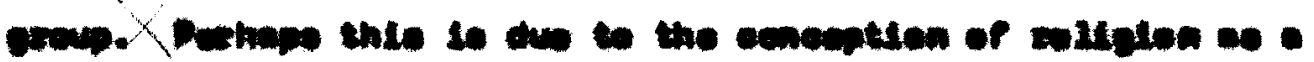

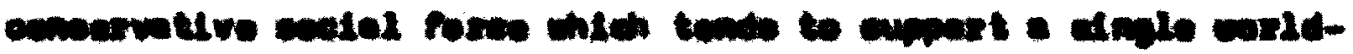

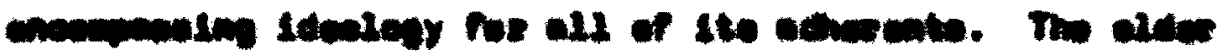

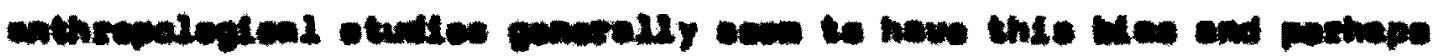

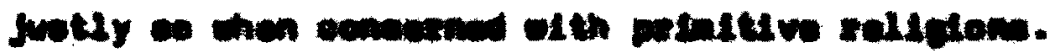

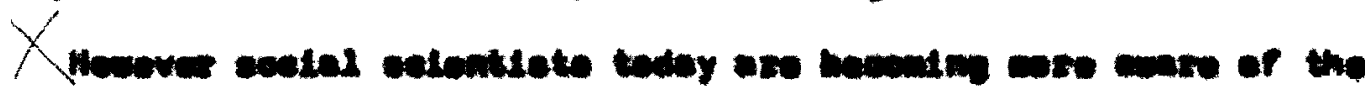

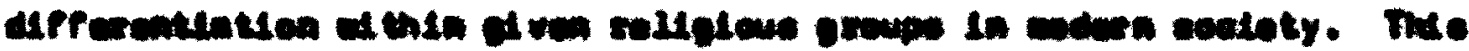

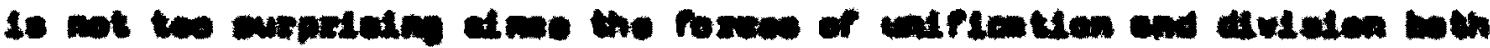

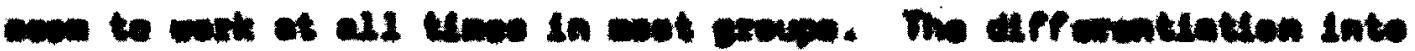

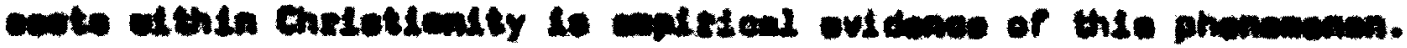

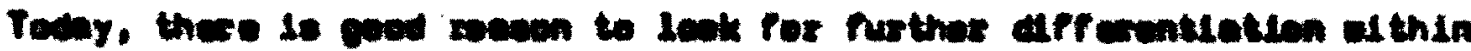




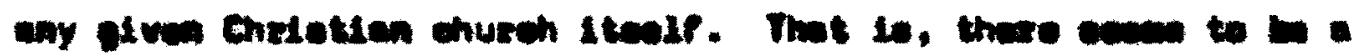

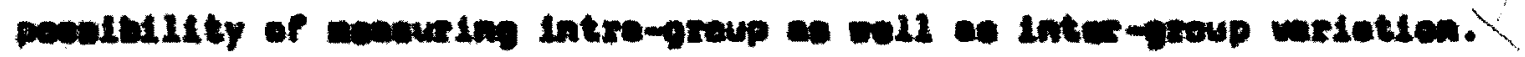

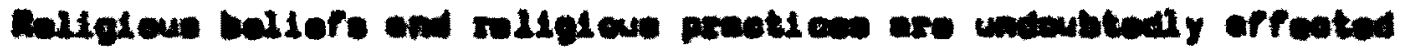

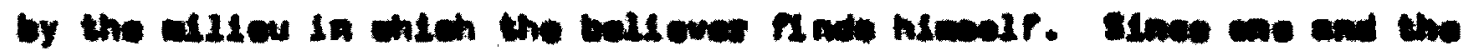

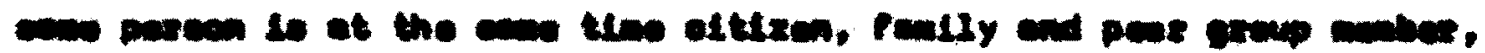

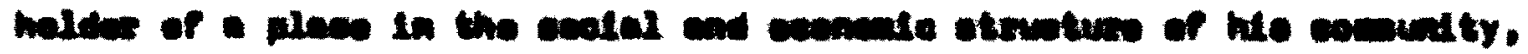

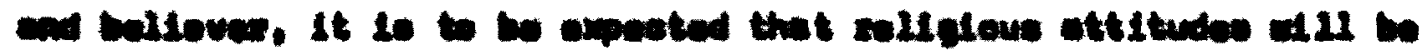

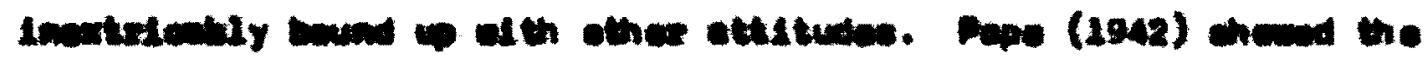

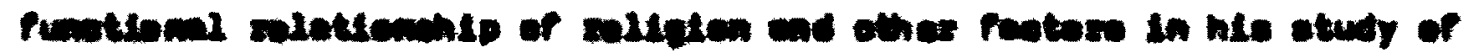

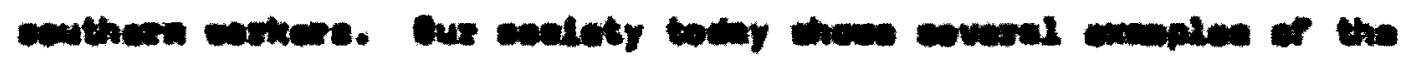

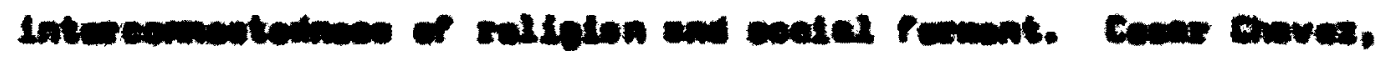

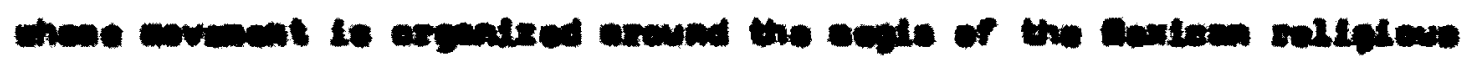

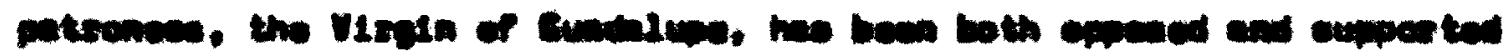

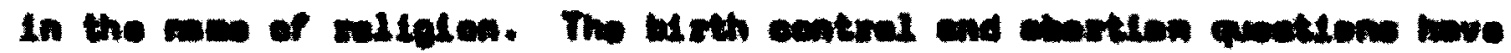

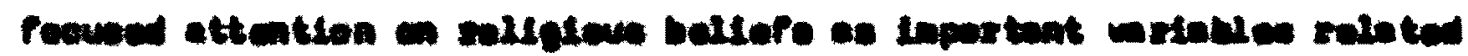
te there prehiom.

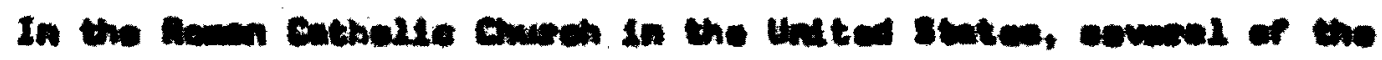

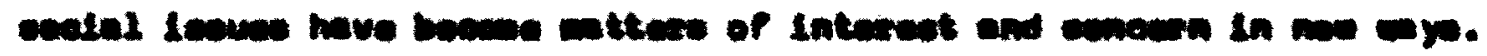

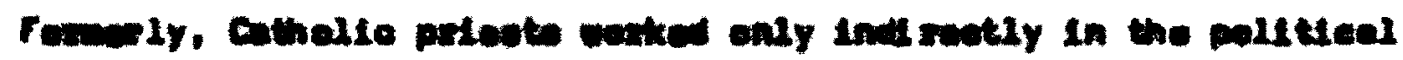

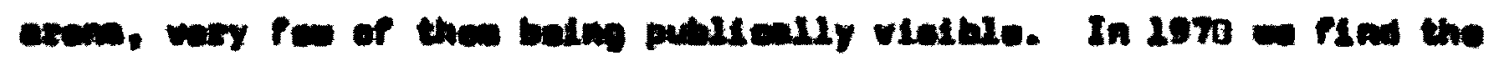

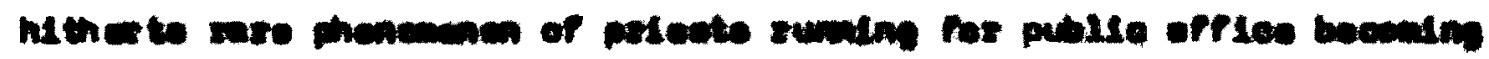

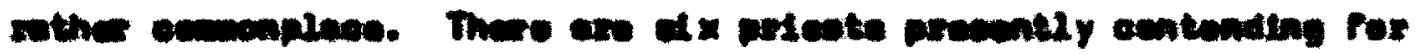

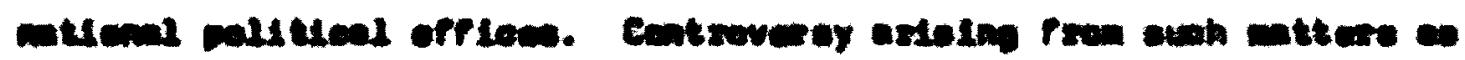

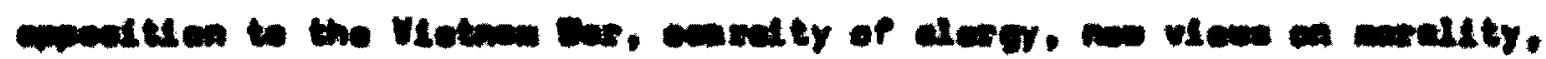

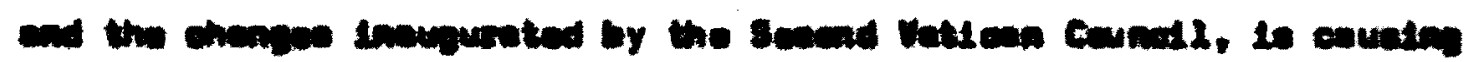

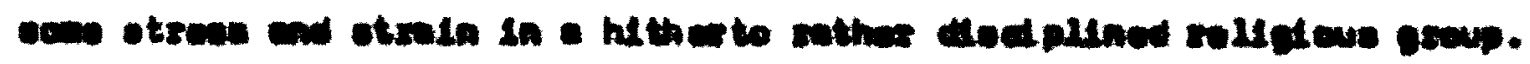




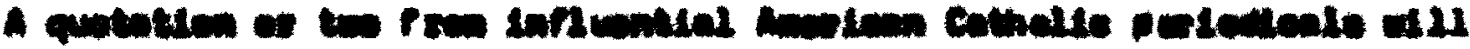
nalp douming bis rot.

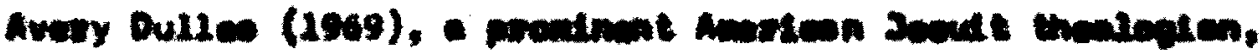

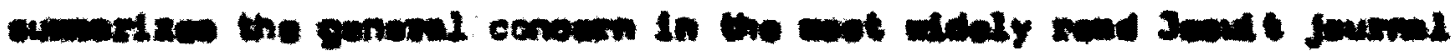
or minten, suntery

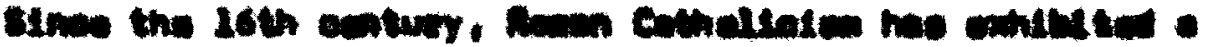

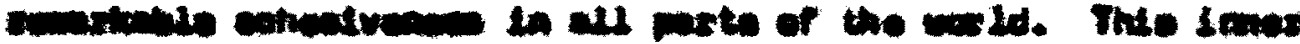

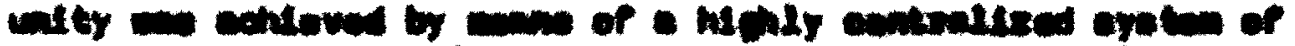

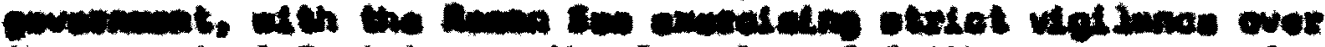

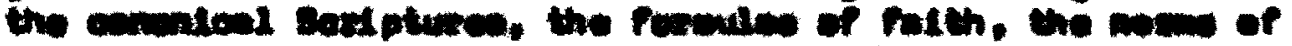

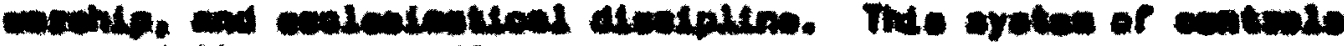

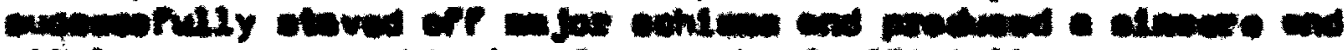

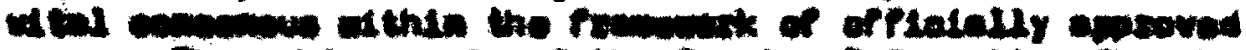

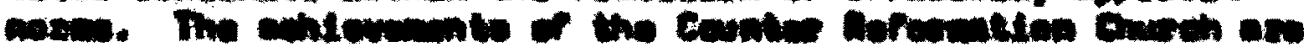

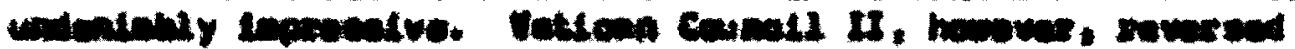

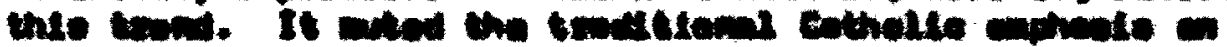

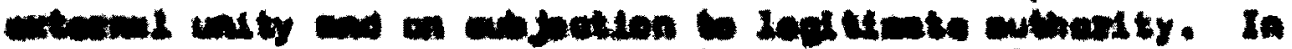

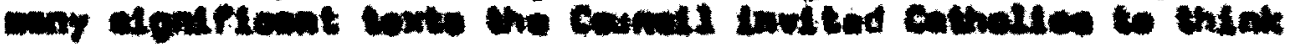

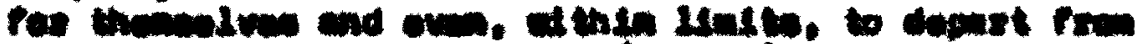

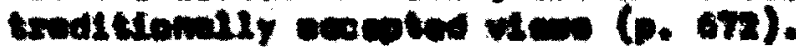

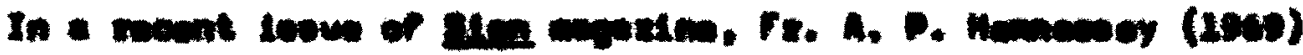

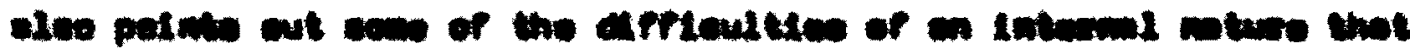

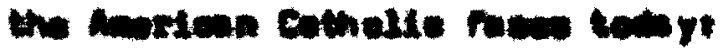

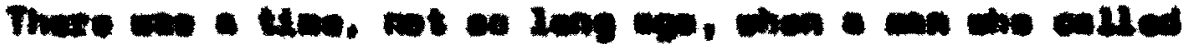

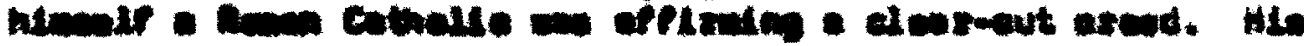

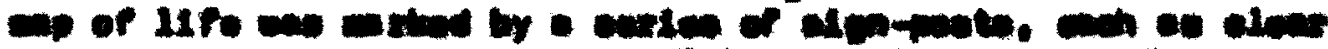

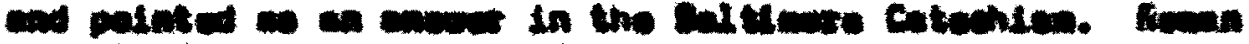

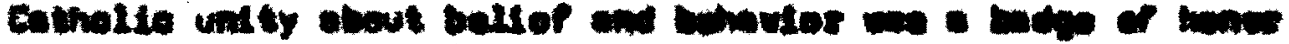

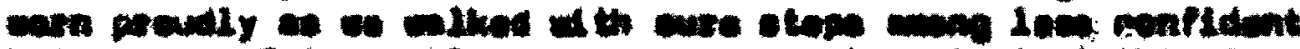

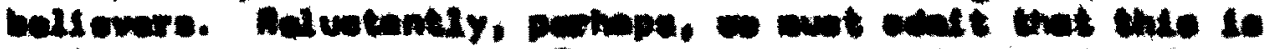

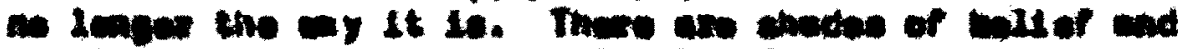

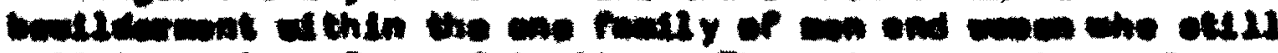

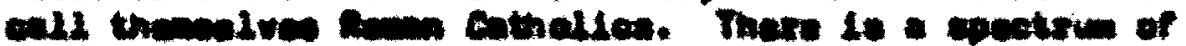

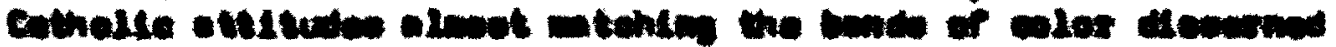

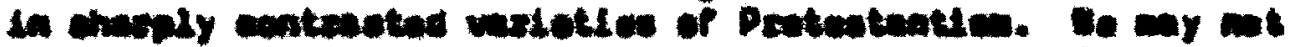

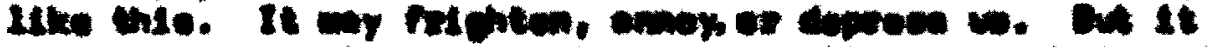

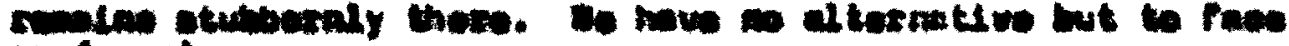
14 (6. 6).

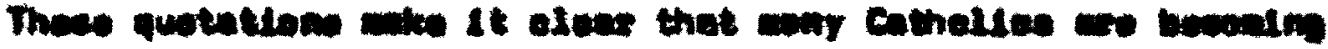

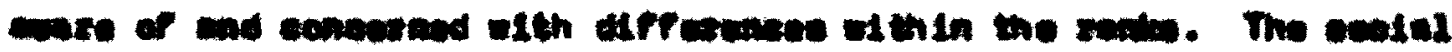




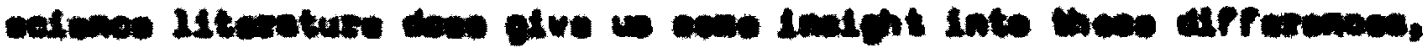

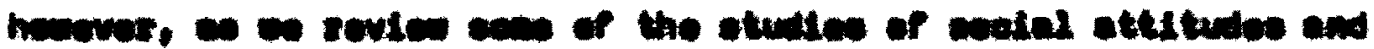

thats owrintes.

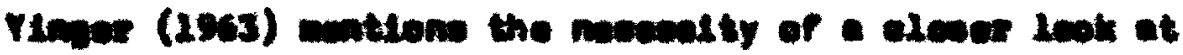

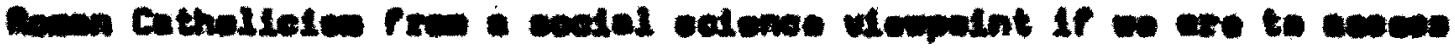

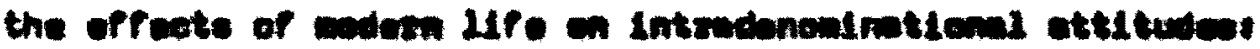

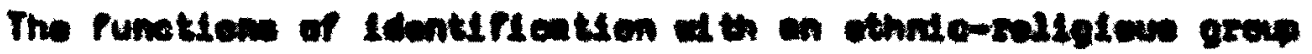

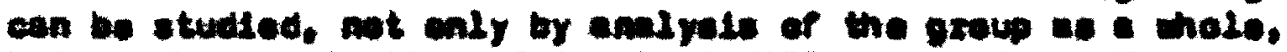

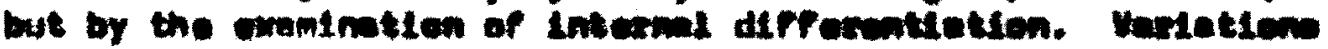

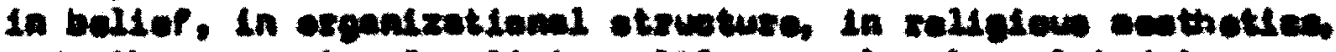

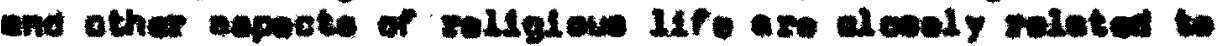

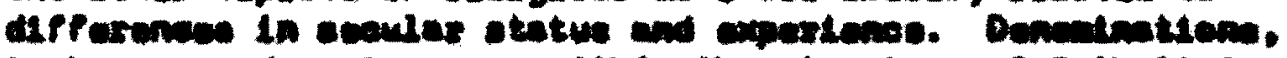

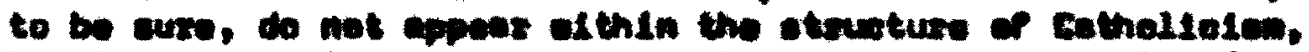

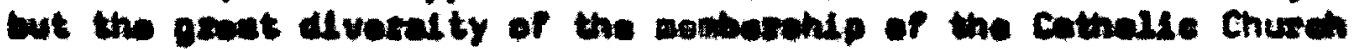

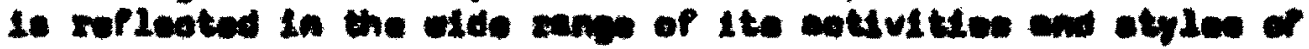

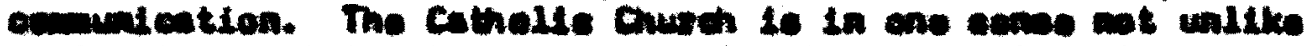

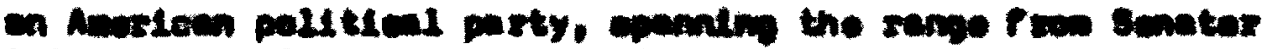

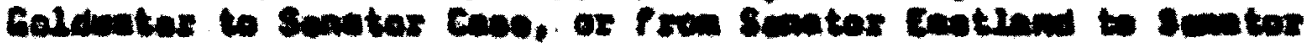

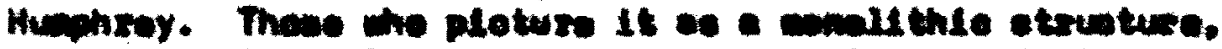

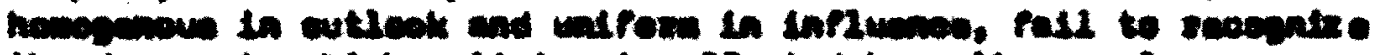
the dogres to mich milglen is apreated by eulture, alses, ceoupatien, cheationd Lovel, and ather ceoulas reate. Thore

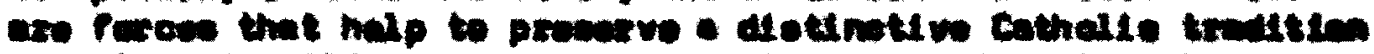

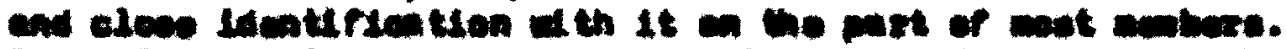

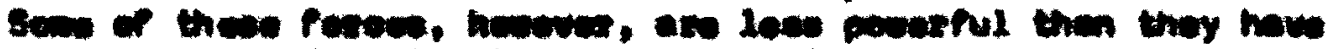

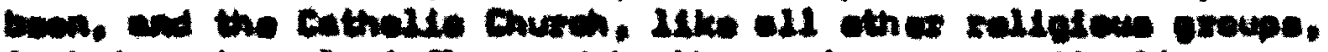

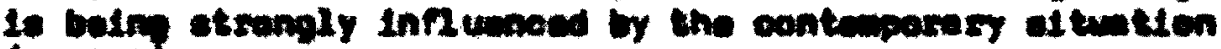
(b. 101 ).

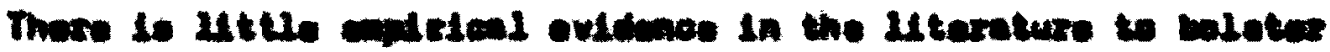

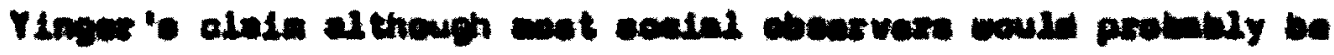

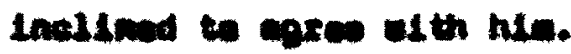

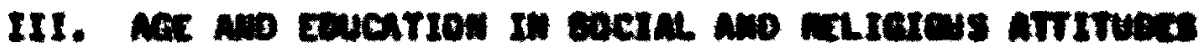

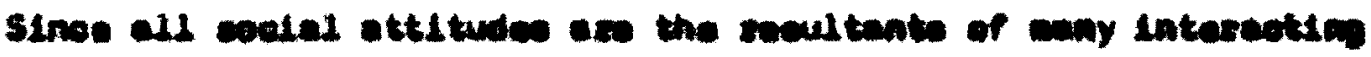

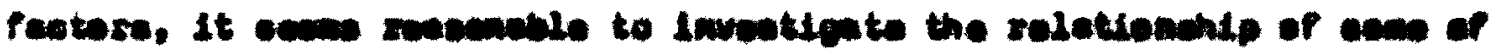

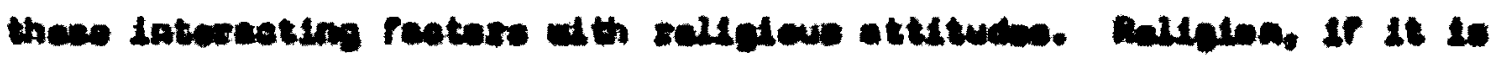

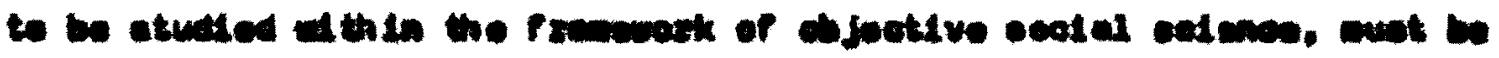




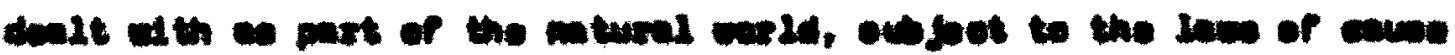

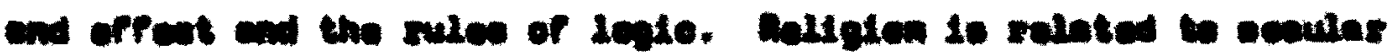

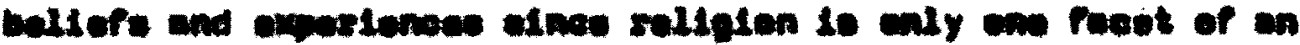

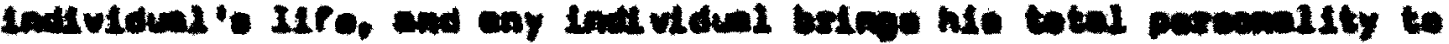

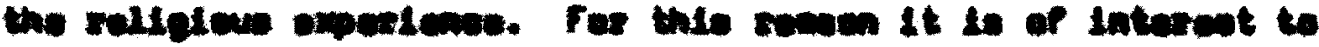

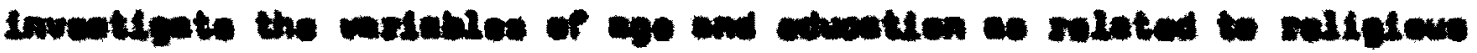

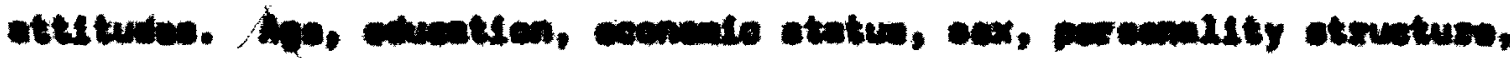

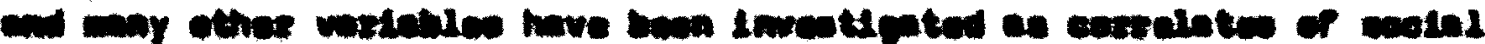

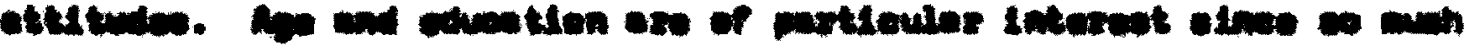

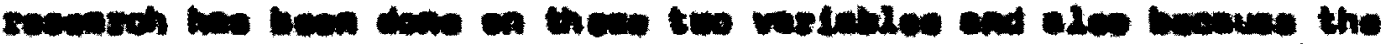

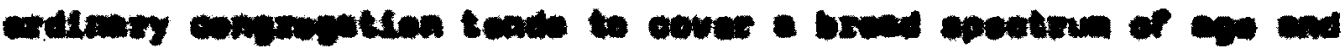

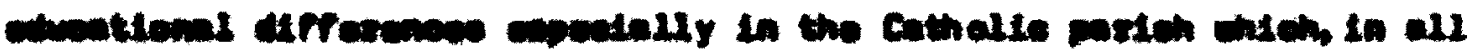

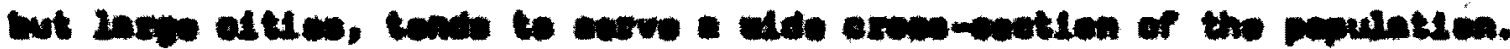

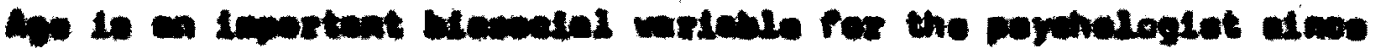

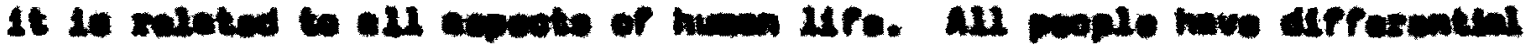

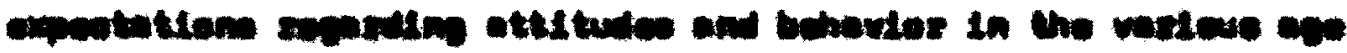

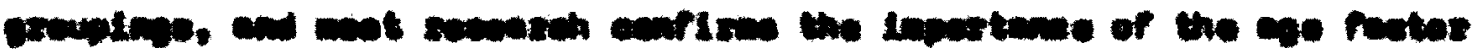

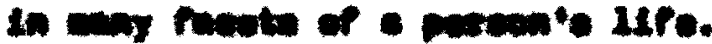

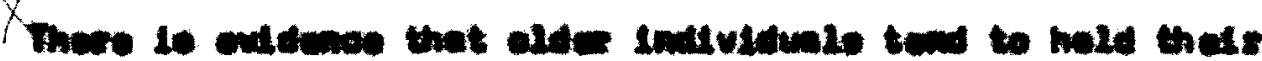

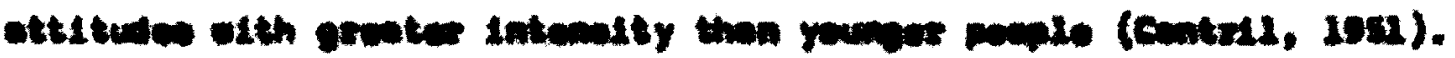

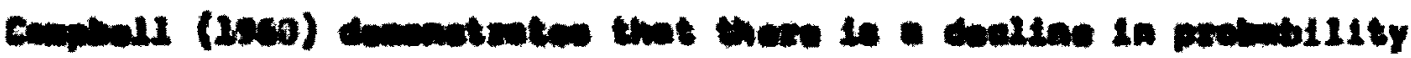

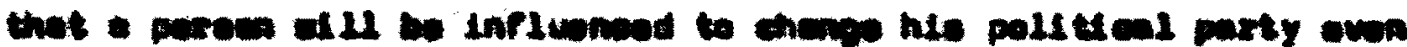

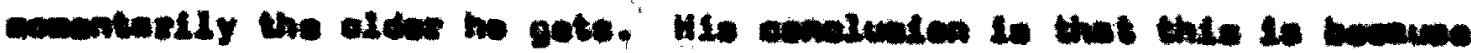

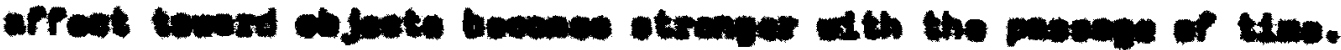

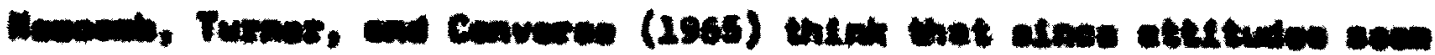

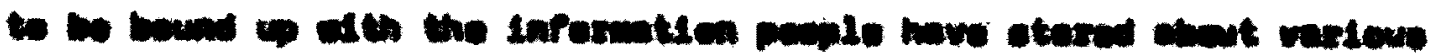




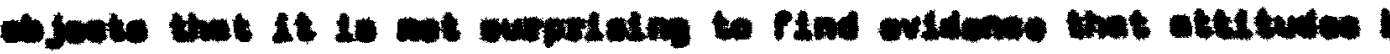

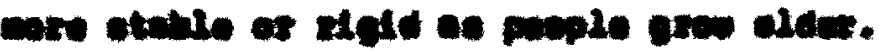

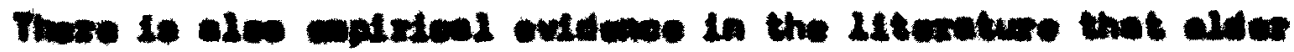

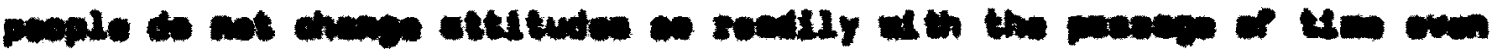

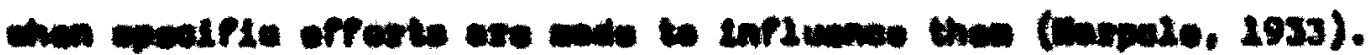

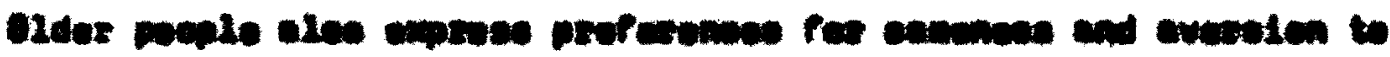

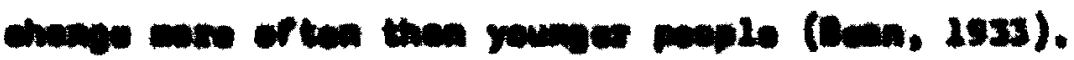

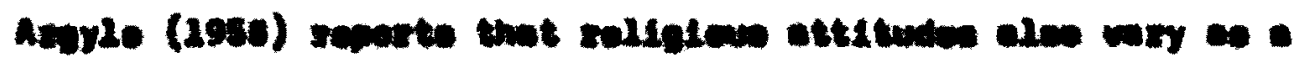

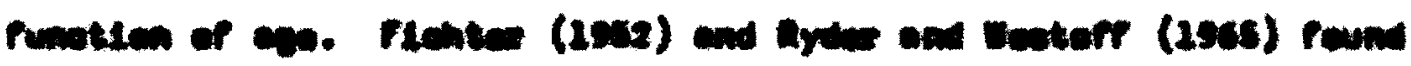

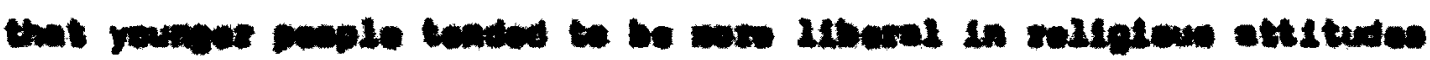
then alces ponis.

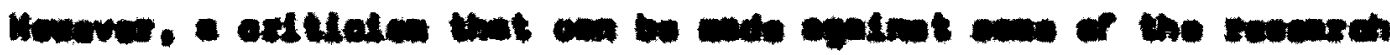

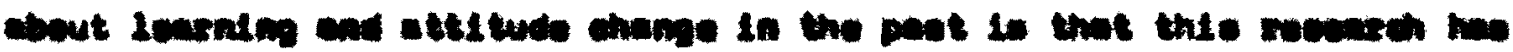

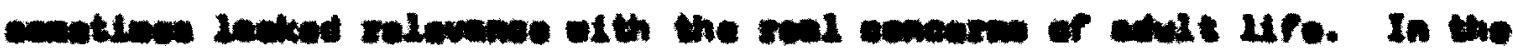

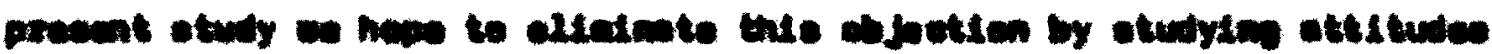

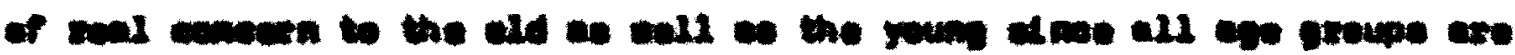

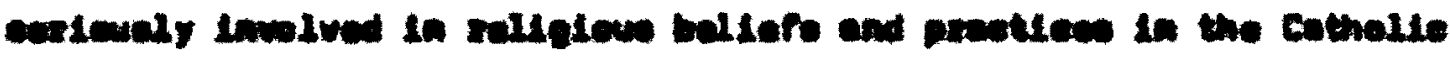
Chanch.

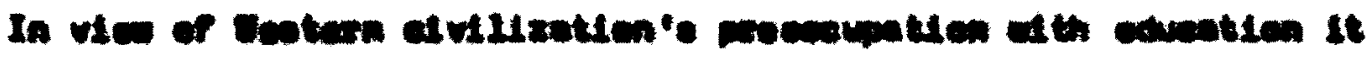

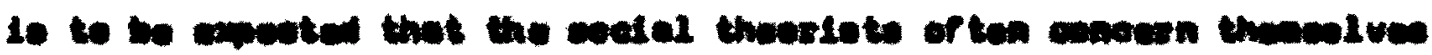

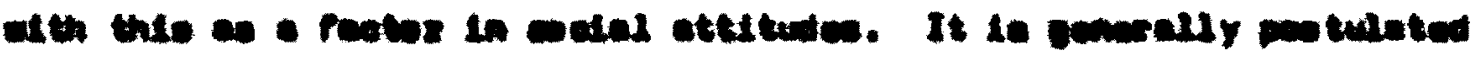

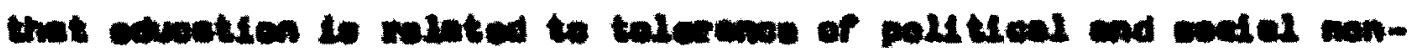

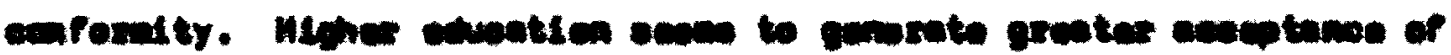

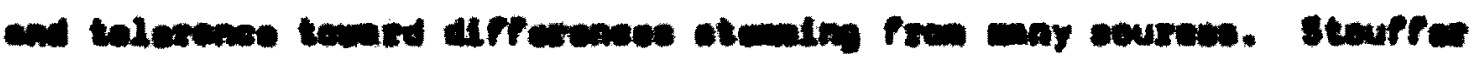

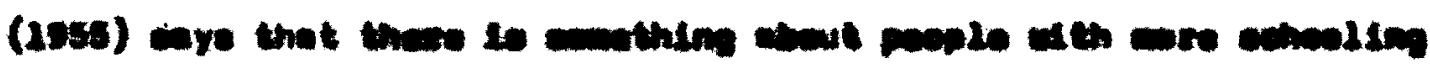

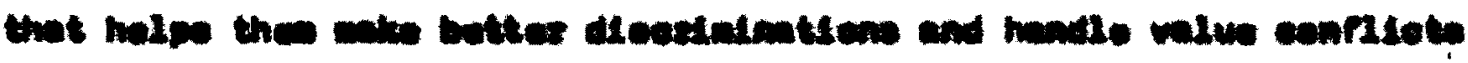

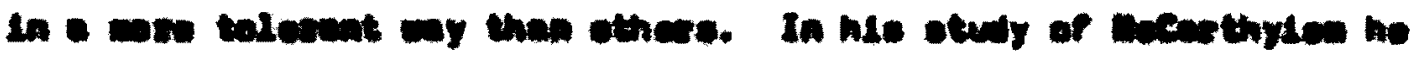




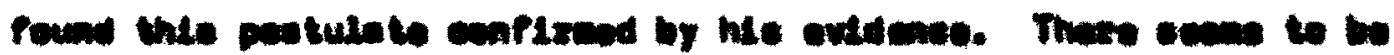

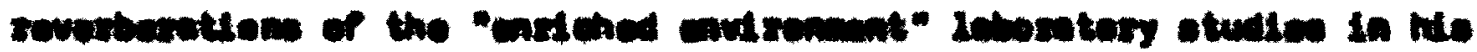

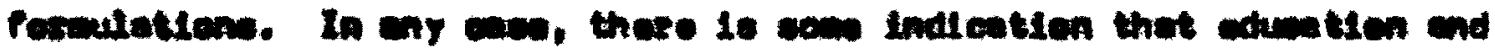
Lberellen eovery pooltivaly.

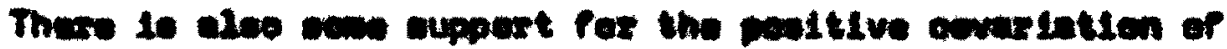

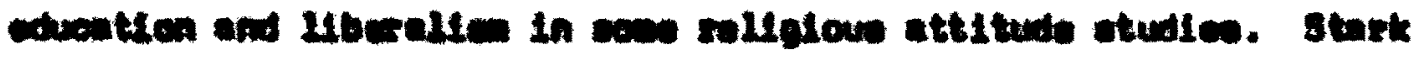

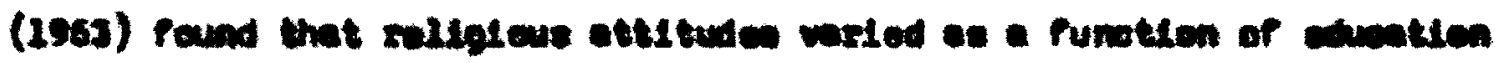

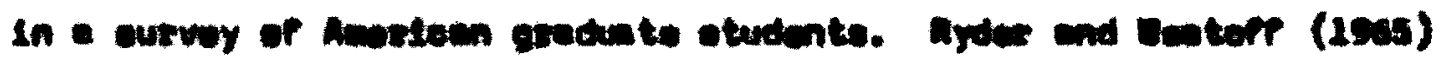

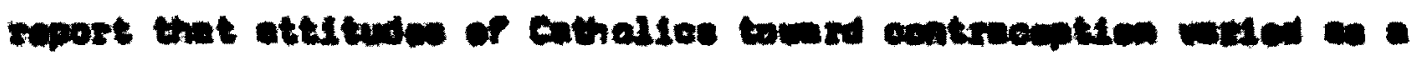

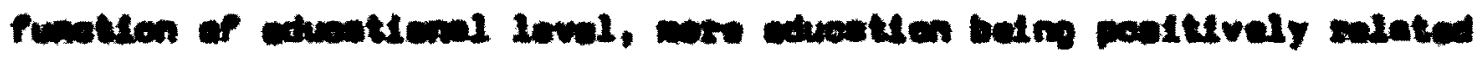

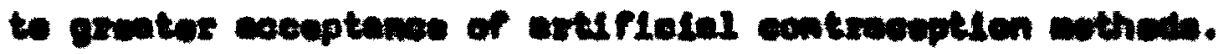

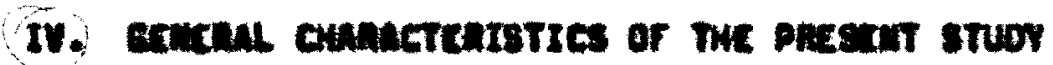

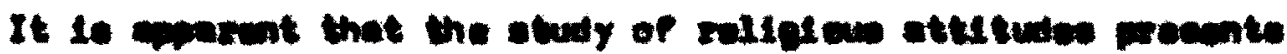

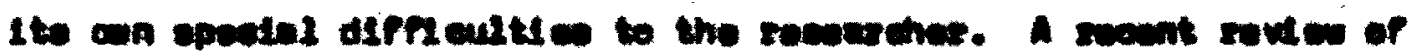

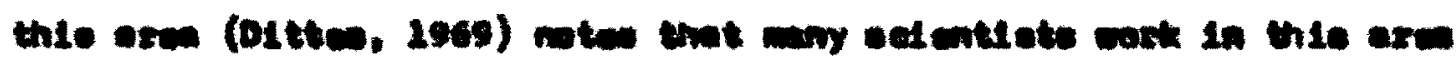

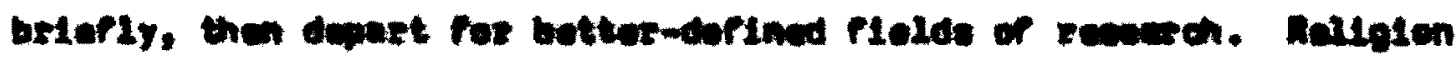

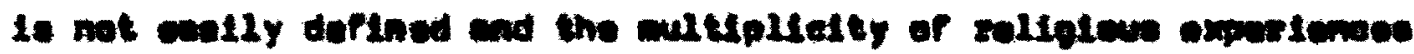

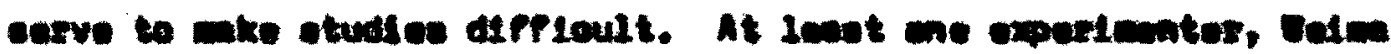

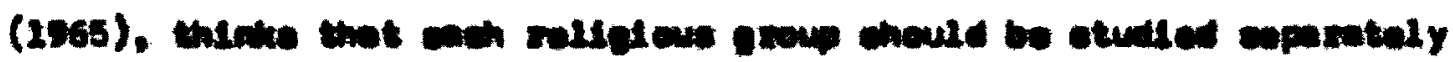

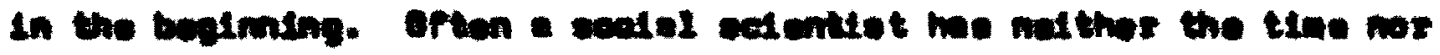

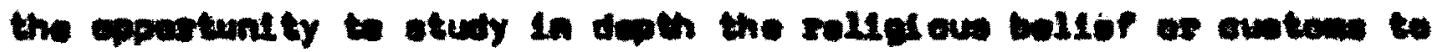

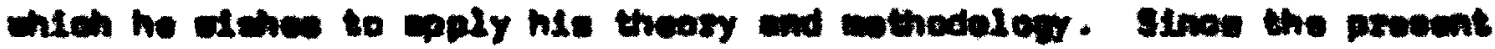

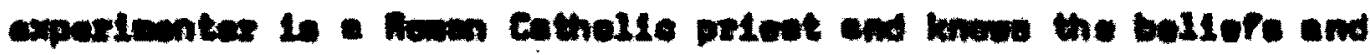

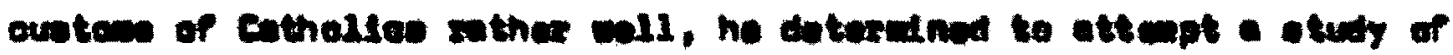

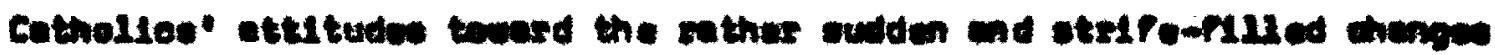
toklm pleve in the Nemen Catholte Chuseh teday. 
The varieblee of age and education were chasen ea thees of particulax interaet. The population prom onsoh the ecaple in the present etudy 10 drewn conalete of easll periehes, and each parioh containe people of ell age and educetional levele elnce there le only one parioh in each locetion. Thus there le no poselbility of poople of one oducotionel lovel or age lovel worehipaling and lnterecting eo - aeperate religious group. The population thue secme to be a eultable one in uhlch to teot the hypotheole that religlowe attitudea vary ol th ege and education.

Some studios are posolble only at aiven timee. Stoupfor's (1955) etudy of MeCarthylea and Foetinger, Rlocken, and Schachtor'• (1956) etudy of rellgloue criele could only be done at times when the phanomen to be Inveetigeted offered themeslves to the obeorver. Todey's tusmall over changes in the Reanen Catholle Church ecene to offer this type of opportunity to the precent reesarcher. Chenge 1o the metter of oreetest concern in the Catholle Church et preaent. Change wll be operetlonally dofined for the purpeses of thie Inveotigetion es eny altoring of bellef or practice emong Cathollce that hae taken plece in the paet twenty yeare or 10 eugsested for the foresencble future.

Since the changee taking plece cover meny phasee of Cathollo 11fe It wee considered poealble to conotruct a ecele of Iteme related to these changes and use a pereon'e ecore on thle ecole se ecme Indicator of hle ettitudee toward theee changee. For the purpoese of thie atudy - Lberal wae operationally dofined as one the favore a ahange and a conservative ese one who opposes chenge. The llberel-conservative continuum le often one uaed in religlous ettitude reecarch (He1m, 1965) and lo eapeclelly eppropriate elnce religlon le ueually conoldered 
- conoervative eocial force. But thle io not elvaye true end religloue backing to often given to liberal and radicel demende for cacled change It we coneldered of prectlcel and theoreticel intereet to doterifin if heterogeneity of ettltude towerd religioue objecte exieted in ordinary conprogetione coverying w th ege and oducation. If so, this would

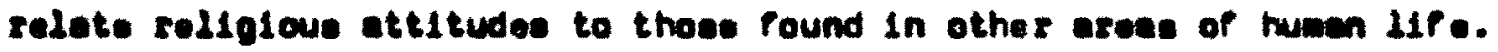
It Io thu the general purpose of this etudy to cepltalize on the present crlele of change w thin the Rown Cothollc Church and doternine

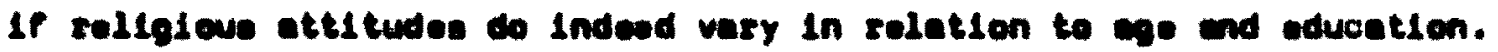
It wes comeldered deolrable to hald the dogree of rellgious apridiation and the knmbledpe factor a oteble wo powfle to teot our

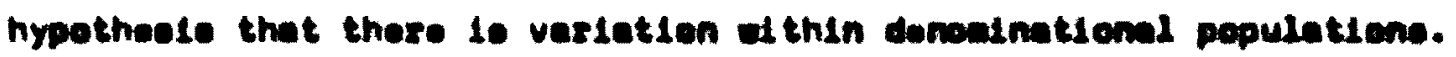
Thie to eccemplishod by oelocting pepuletion of requidrly churchottending Ëetholice. People often label thomelves as Catholles aimply

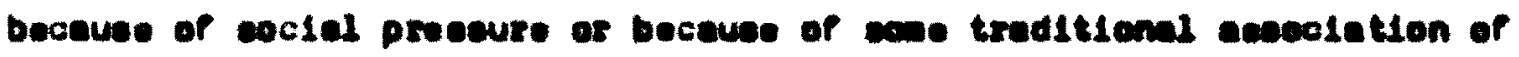
thel: feally wth the Cathello Church. Swoh affilletien och hardly bo eccepted es a purentes of true Church menberohlp es socetanes of Cacholle bellafe and prectices. To bo wre, ohooelng a church-galng population comot be taken no proef of the abjecte' oumatimente or bellefe, but it et least esurse ws that our soependonte are in touch ath the changes occuszing in the Cothelle Church and that thoos changes have ocme Inpact on thels 11vee, and hance ase relownt to then at leat In cre anwe of the tex.

Statlotionly, Lielting the population to churoh-ettendere does xemove eame of the veriebldity that would be found if the population wore deflnod ae conoleting of enyene whe cheos the label of Reman catholle. Sut thie otudy 10 not intereoted in wrichility of attitudeo 


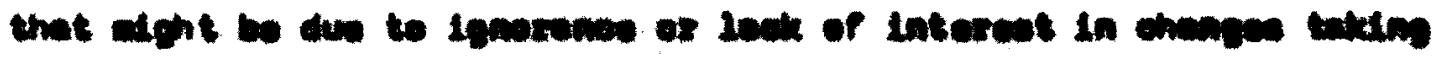

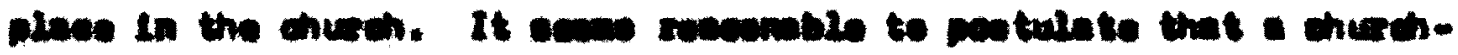

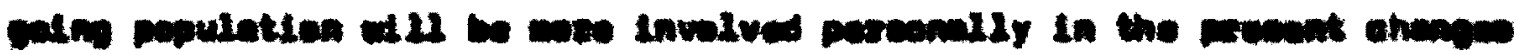

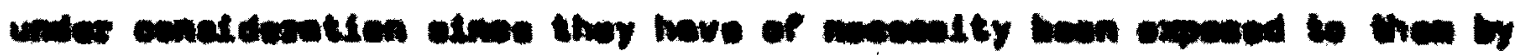

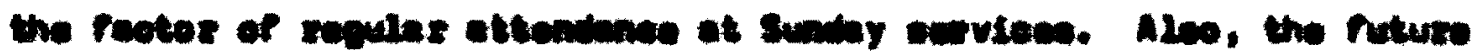

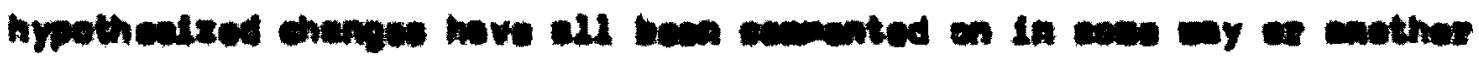

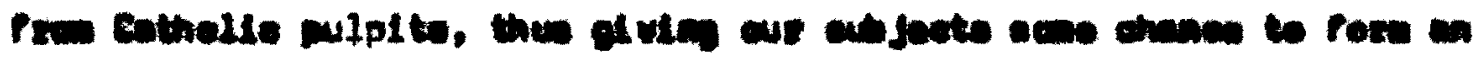
stritude conocation thes.

\section{v. exurancy}

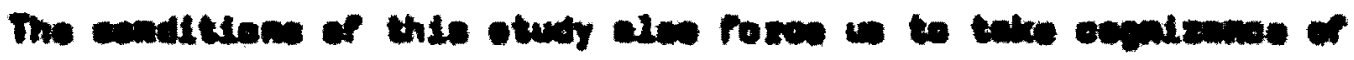

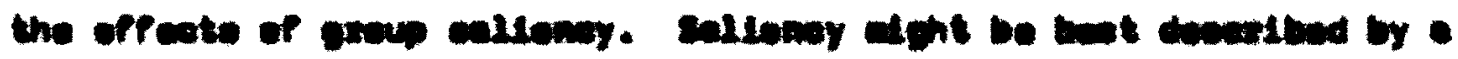
potatien frim kelley (19as):

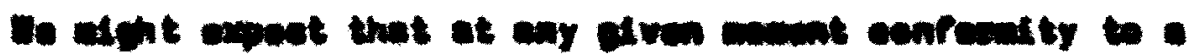

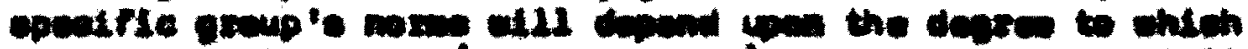

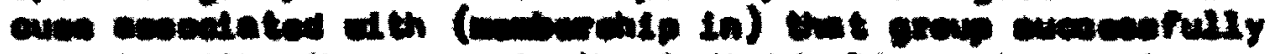

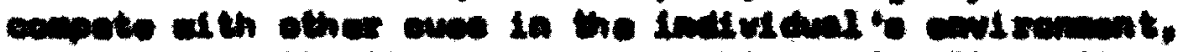

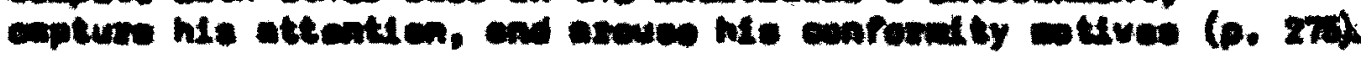

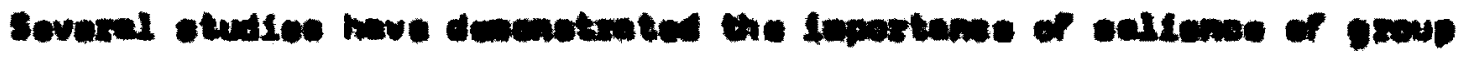

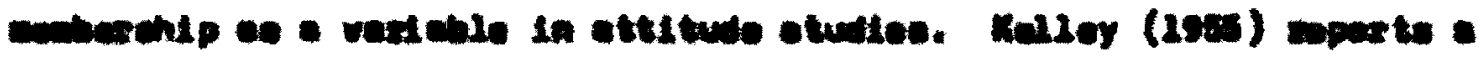

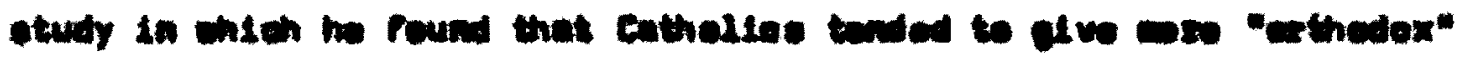

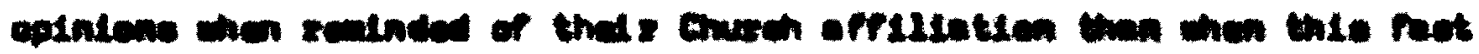

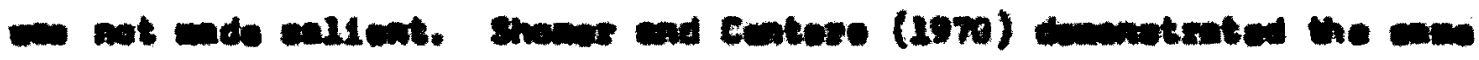
menomemen.

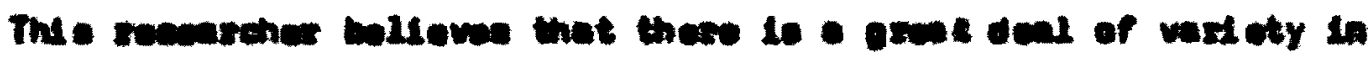

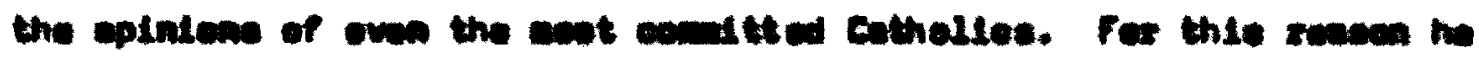

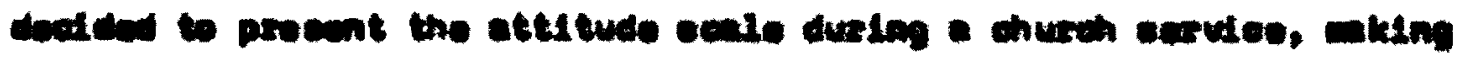

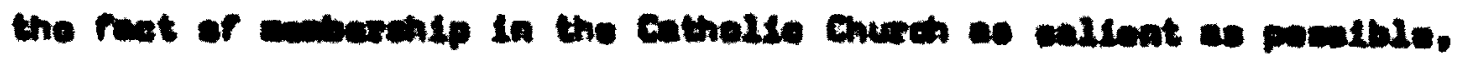

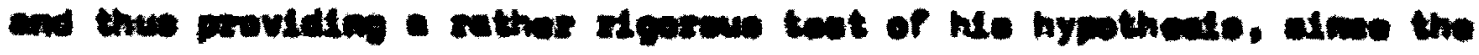


16

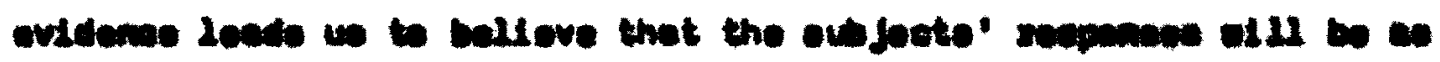

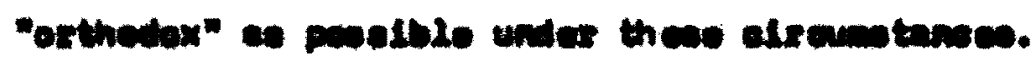

valker and Firotto (1965) also soport that the group wamberehlp

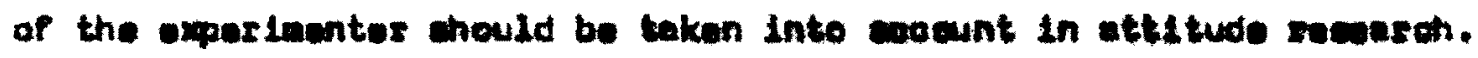

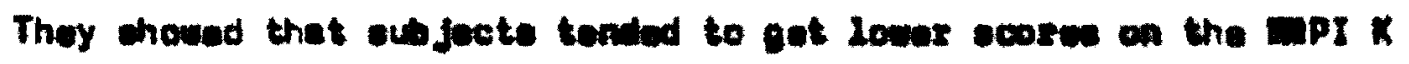

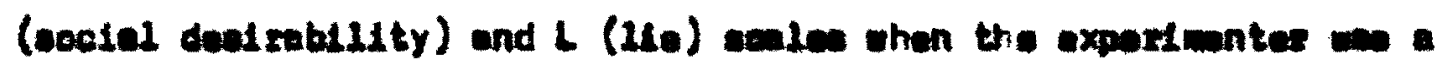

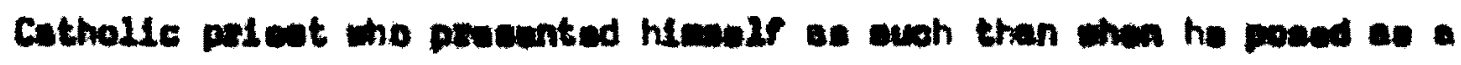
Layean. Aecorting to this atudy the aduiniatretion of the instruent by a priest thould both hals to inerwese the rellability of the scoses

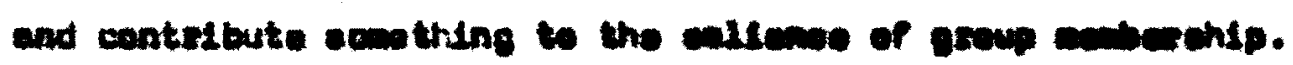




\section{canpren II}

\section{mocrount}

\section{Amoos - Dan 1}

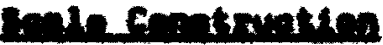

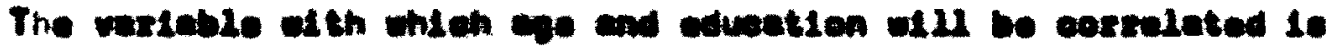

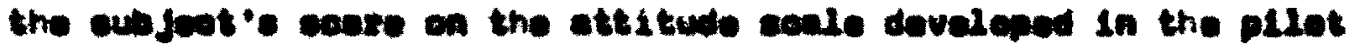

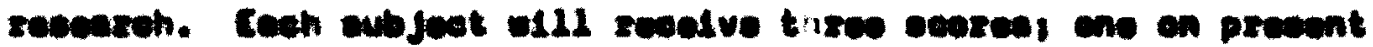

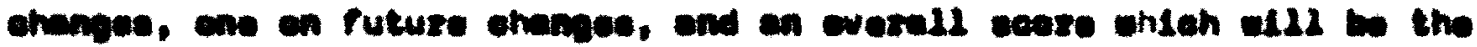

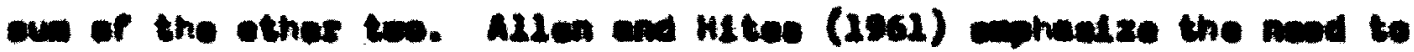

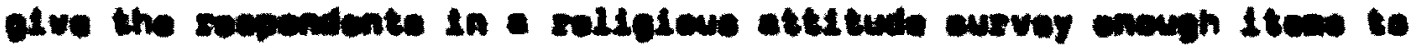

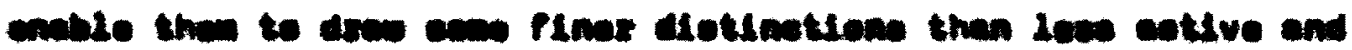

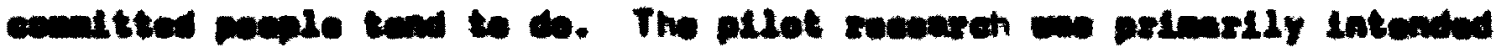

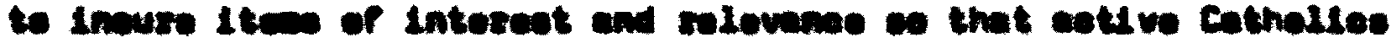

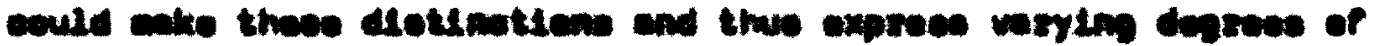
ancoptames of manes.

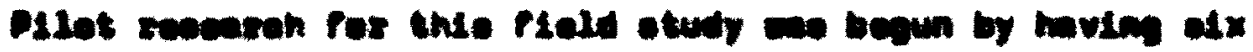

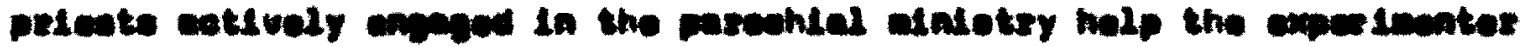

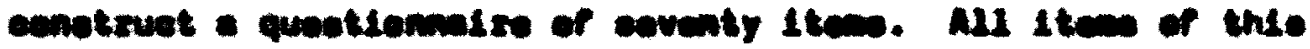

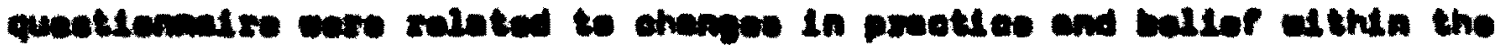

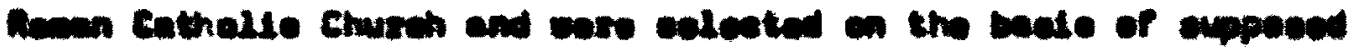

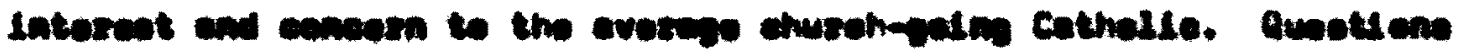

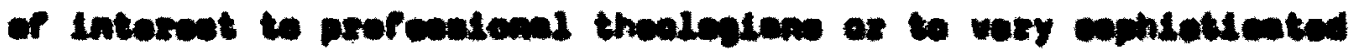

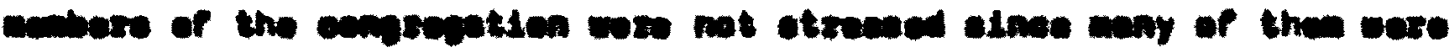




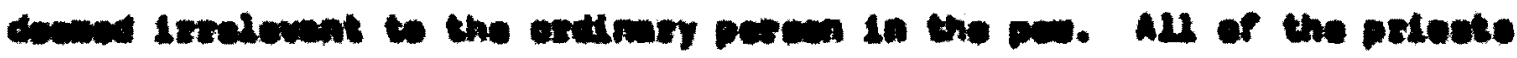

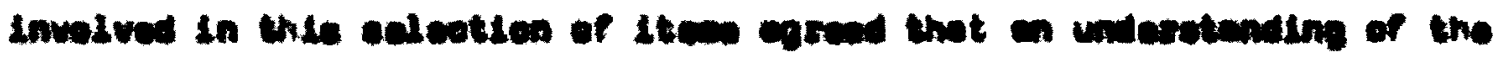

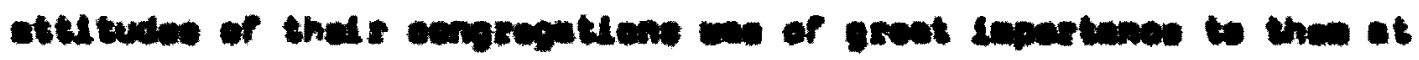

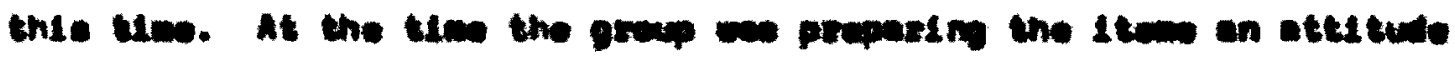

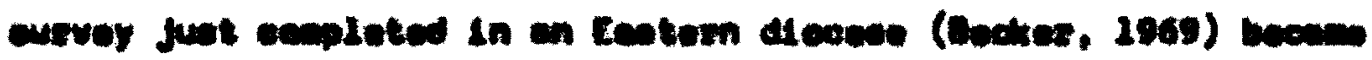

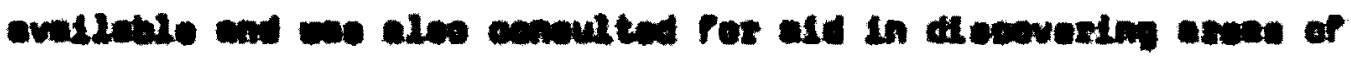

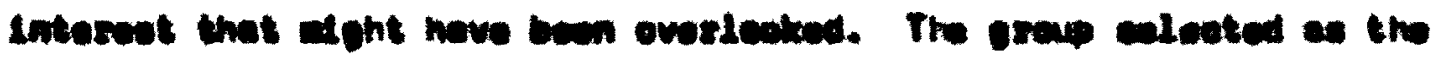

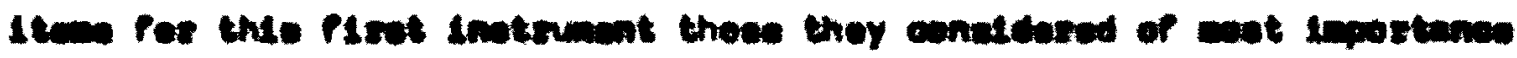
te thale nompotions.

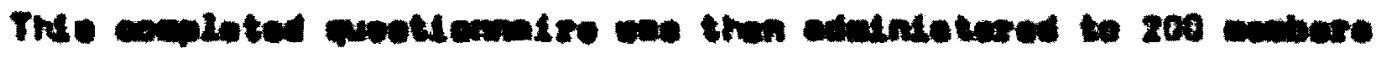

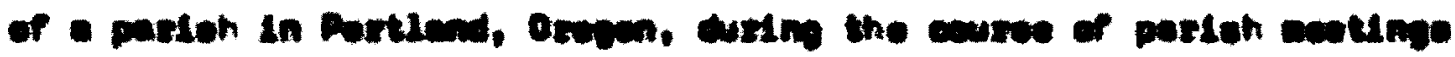

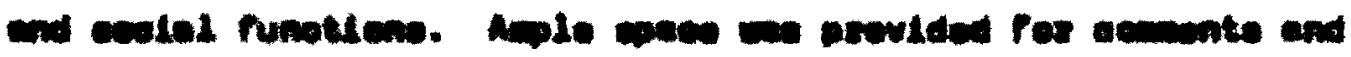

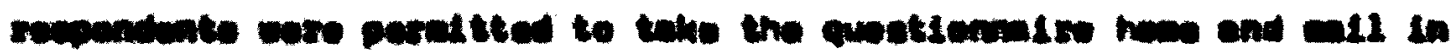
the solum if thay atehed.

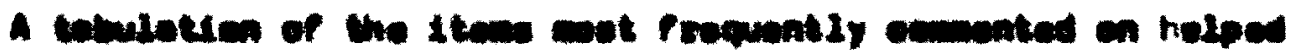

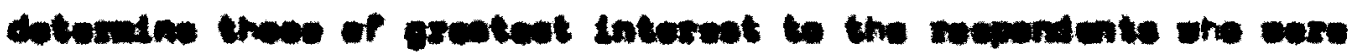

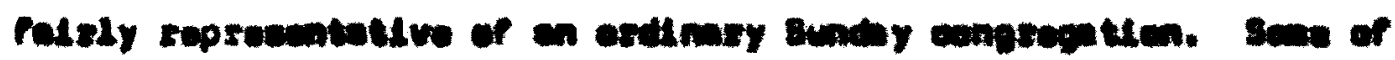

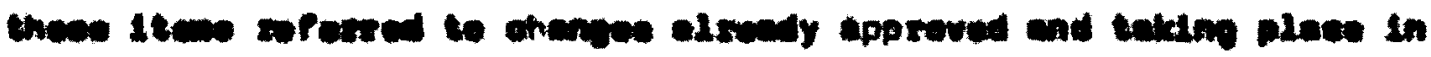

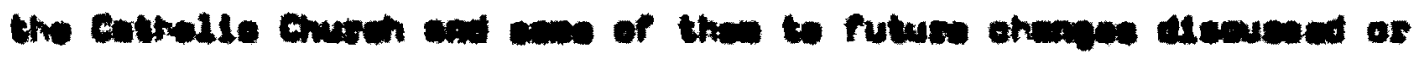

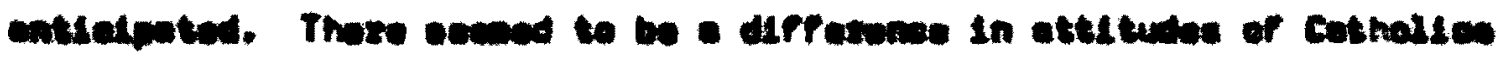

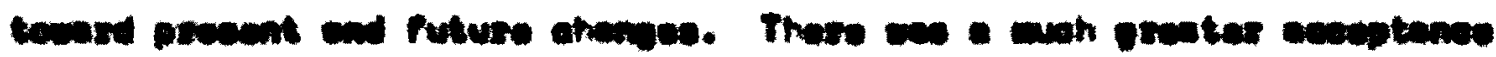

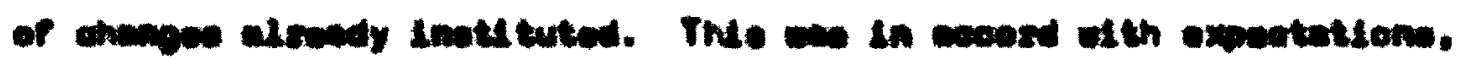

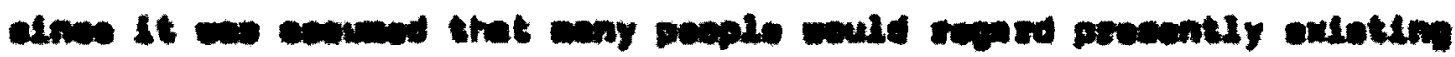

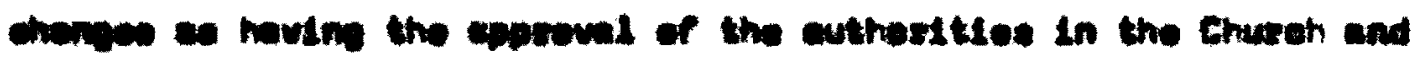

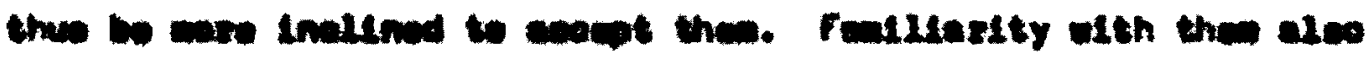

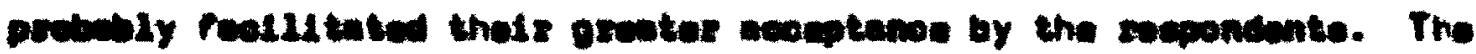




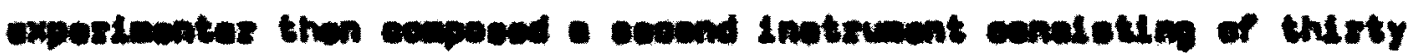

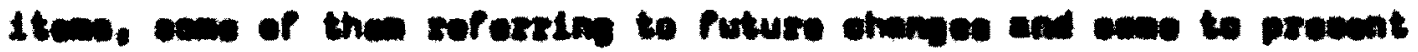
ones.

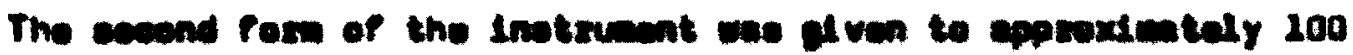

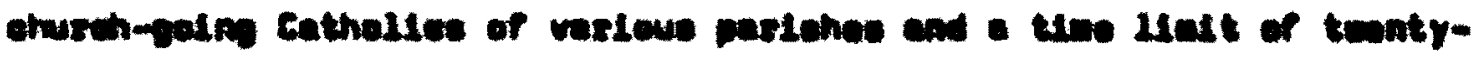

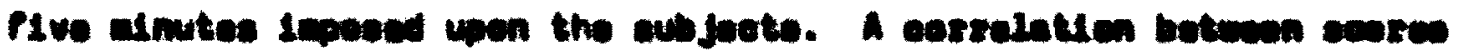

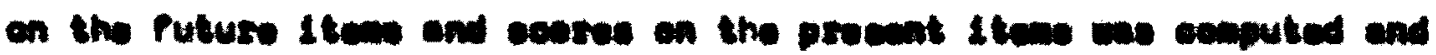

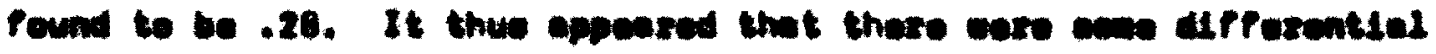

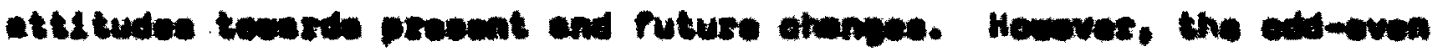
epllthalf correlntien on the ocale weo enmuted and found to be in

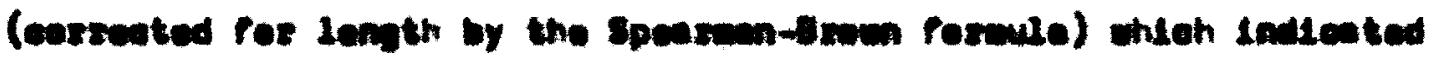

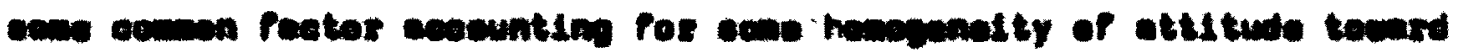

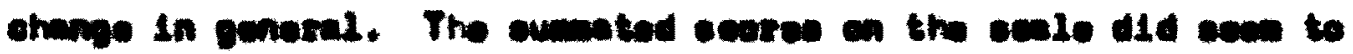

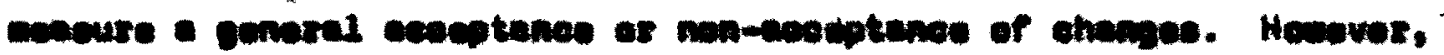

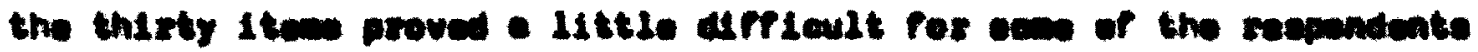

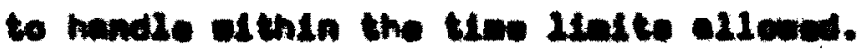

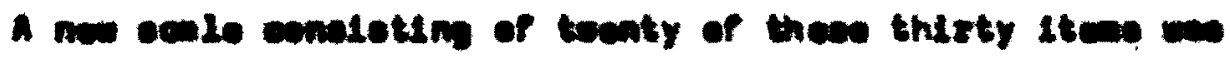

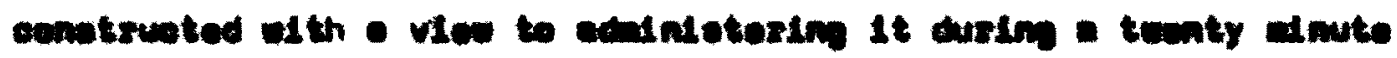

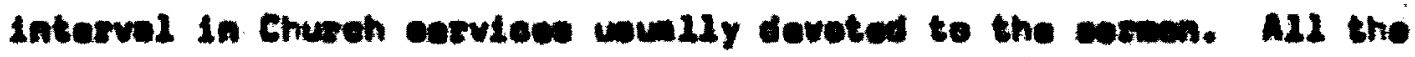

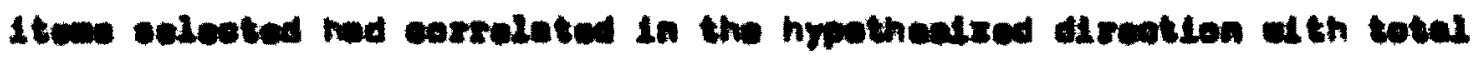
seose on - polnt blearted correlation sun on the wta of the coecond etubly. Ten of these 1tem (1tam 3, 5, 7, 3, 22, 21, 16, 17, 18, and 20 .

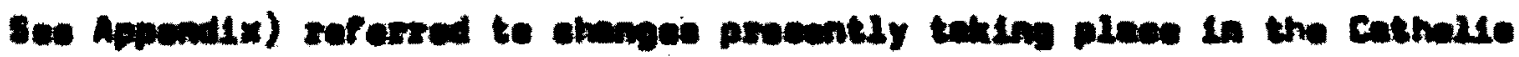
Chureh. The other ten (1tam 1, 2, 4, 6, 8, 20, 11, 13, 15, and 29)

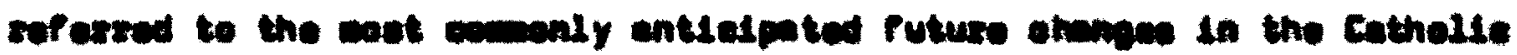

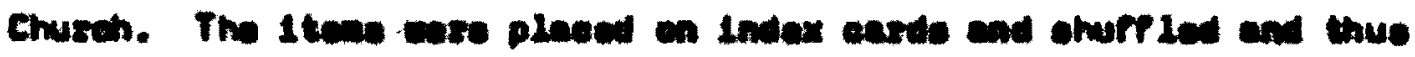

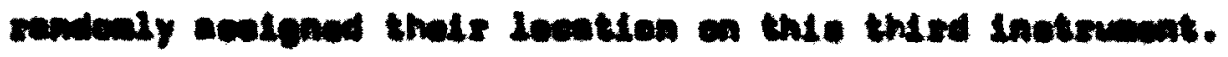




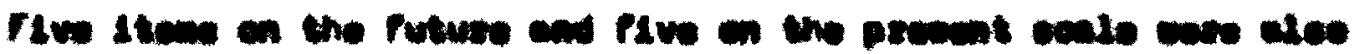

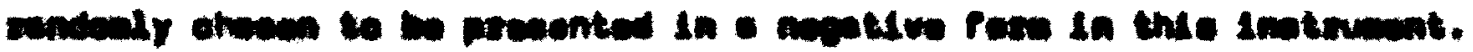

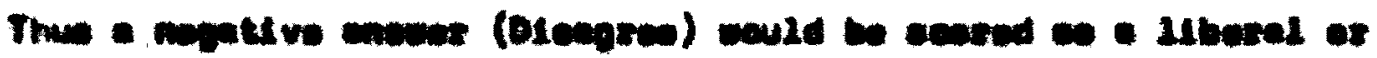

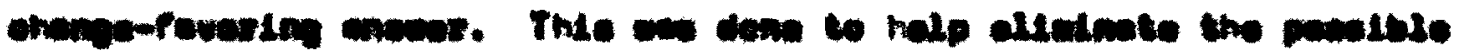

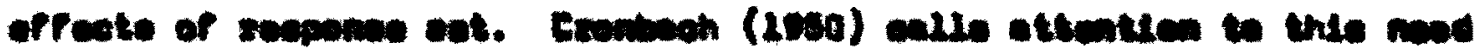

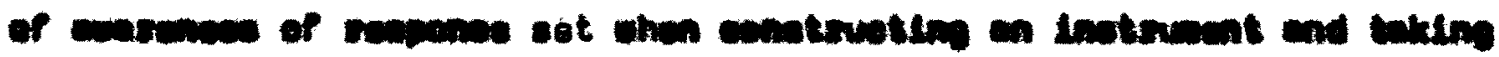

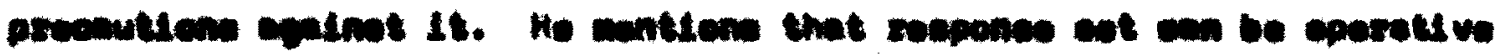

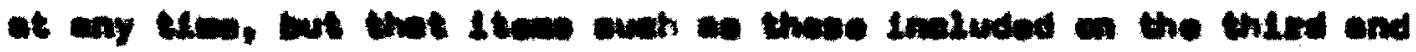

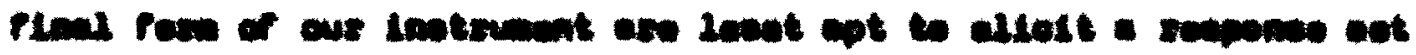

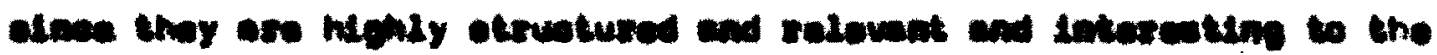
reserenimimat.

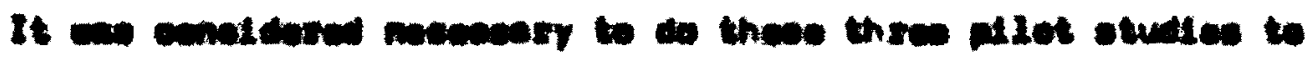

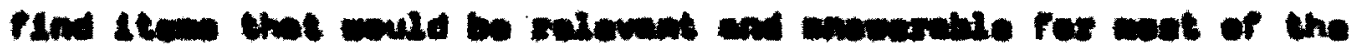

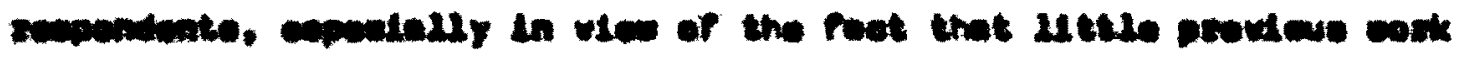

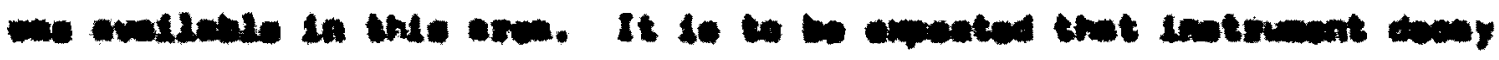

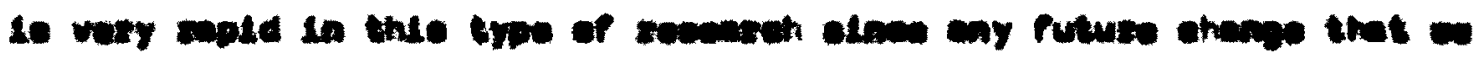

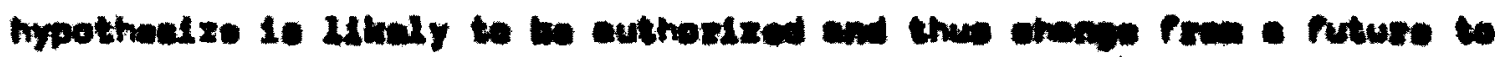

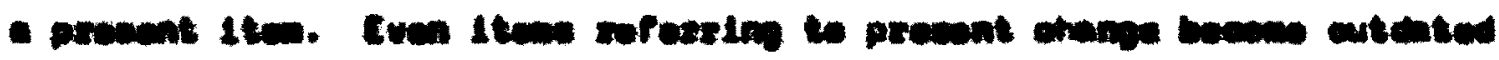

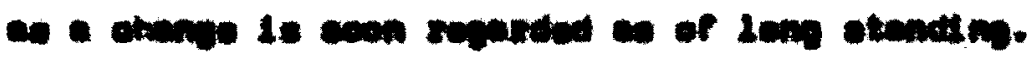

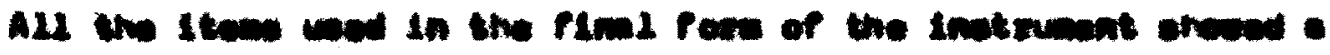

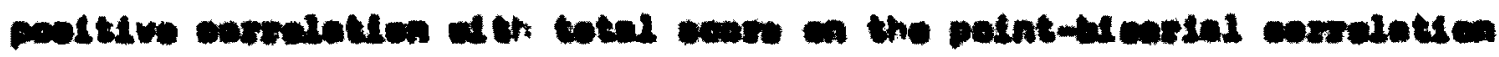

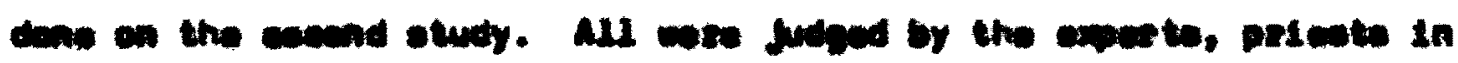

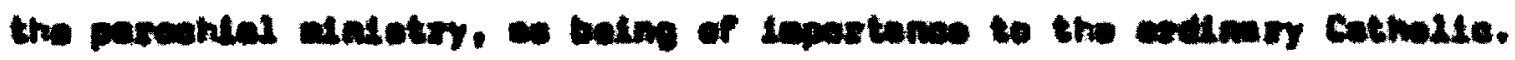

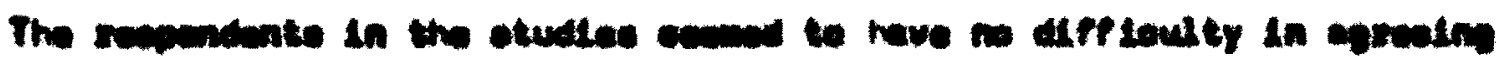

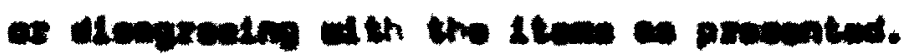

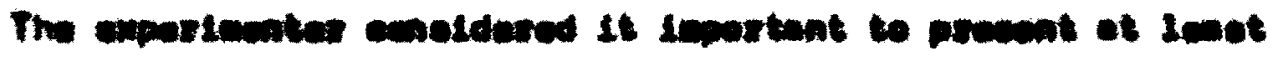

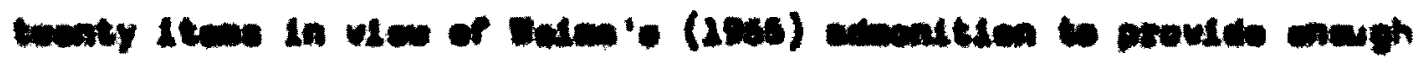


Iteme about chempe to peralt good diecrialnation elong the liberalconservative continuum. For example, in the eree of clerical dieciplino wany Catholice are concerned olth the presont ehortage of clergy in Cothollc perishee. This usually saleos the quetion of clesical celibeoy. Three 1teme $(2,11$, and 15) permit the reepondent to diecrialnate a I1ttio in his attitude toeard this future change. Iten 11 morely acke if the chenge to a anried cleroy lo agreesble to the reopondent. Iten 2 eake if a reopondent thinke thlo ohange would reoult In good eervice to poople. Ite 15 incerporetes the wot frequent coment in the plret two pllot etudiee which Implies thet thie change to a warried olergy alght reoult in botter corvice becuee a married clergy would have botter ind ght into feally life. It 1 s secosmable to aseume that a percon might acoept a change to a married clergy even though be does not foreese it a resulting in good or better eervice but moraly beeause he decme it neceserry to the exietence of the Church ae he knowe 1t. He alght aloo think a exried elergy could give bettor eervice or alght not. He could aloo think a earriad clergy would glve better eervice but prefor the celibate clergy he hes greun ecouetemed to. Thue all three 1teme holp diecrialnate a respondent's attitude toward this ohange.

The ocmente of esller respondente aleo Indiceted eome cencern over the position of women In the Church. Two iteme tap this eree of change. Item 1 checke the repondont "e ettitude toeard a mindmal perticlpation by wemen in worahip and 1 tem 15 checke hle attitude toward - ronale clergy.

Itwo 16 and 18 both oheck the reopondent's ettitude toord tho wee of autherity in the Church. Iteme 9,10 , and 12 are all concerned 
ath change in wrohip peatloes in the chureh. 1 tree 4,6, and 14

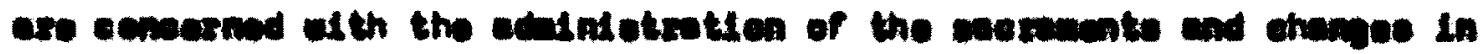

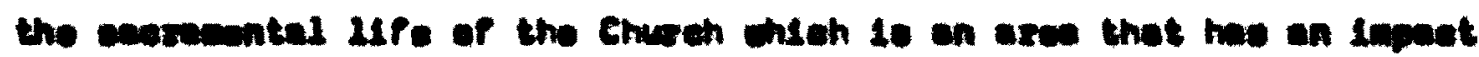
ea the overy day ufe of eny cothelieo.

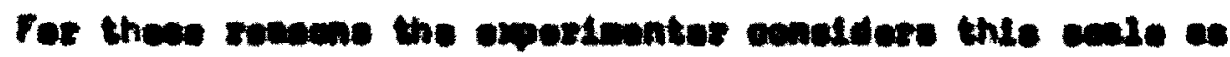

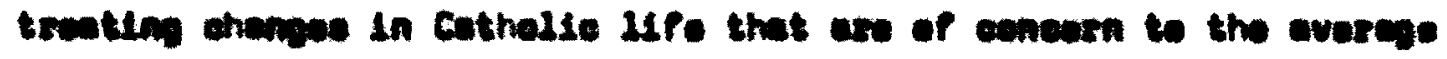
chunch-goles cothols.

Ened Finderint

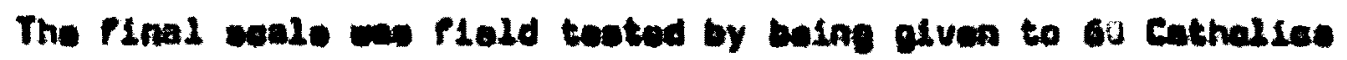

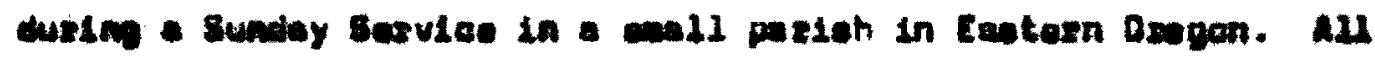

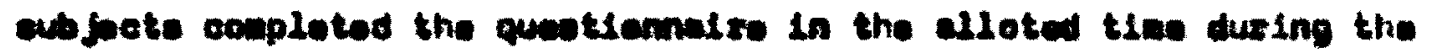
asdde of thi cervice and many expromed interest and acked for a cumery of titio roults.

A eplit-half oexrelation copplasent wes computed for the dat

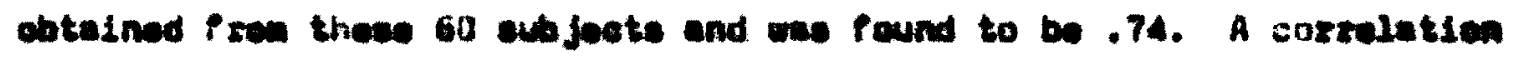

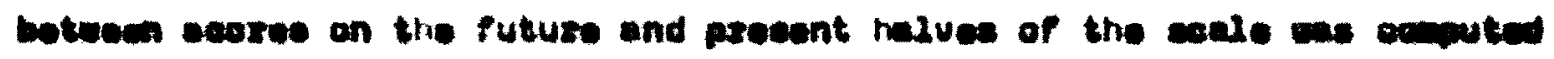
and Pound to be .60.

\section{1. mingos - tant $\mathrm{x}$}

\section{Inthate}

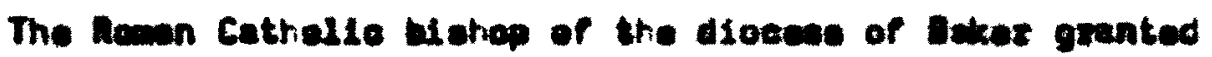

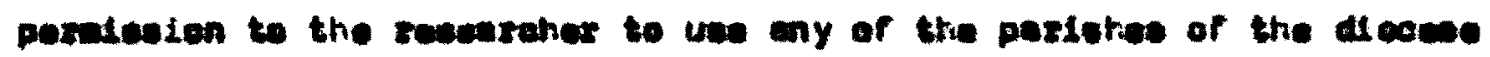
for the etudy. Tro dioese of eakex lneludes noet of castem Oagen end

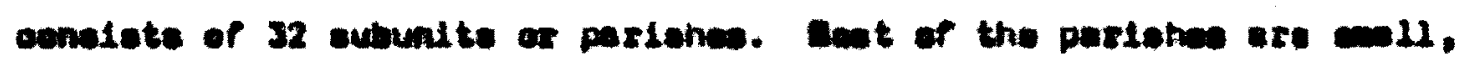

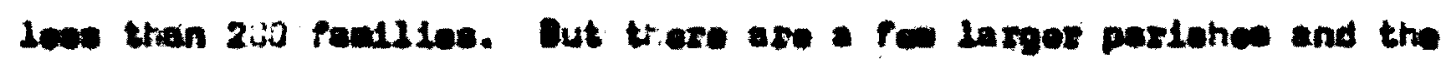

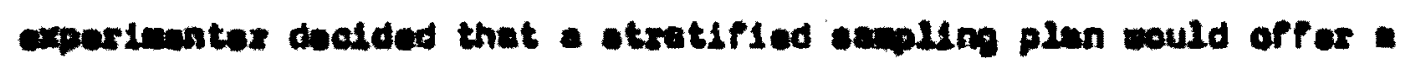


better eample of the population. The pariohes were divided into three

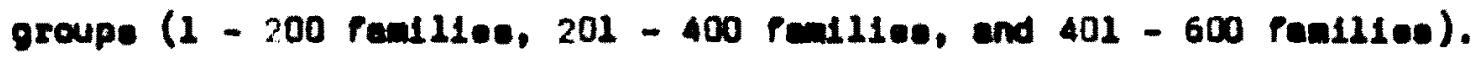
One parioh each frow the two lerger groups and four emall parishes were randomly eel ected ae the eample of periehes to be eurveyed. Thus the ubjecte conotitute a randon stratified eapple of the church-going population of the Remen Cathollc DLocese of Beker. Since there is only one Catholic parion in the emall towne and citles of Eeotern Orogon, all churoh-golng Cathollce of thie erve ere forced to worohip togather thue asouring the experimenter that the variebles of age end education of 11 be falrly representative of the entire Cathalle population of the erea.

\section{Experimenter}

The experinenter, Cathollc priest, offlaieted at the regular Sunday eervices of the parishes Included in the sanple. During the tine In the eervice regularly devoted to a eermon he eoked the uatar to paes out coples of the ettitude scele and penc1le to nenbere of the congregetion. After these had been diatributed the experimenter read the introductory paregraph of the queationme1re to the conpregation. He requented thalr cooperation in complating the 1 teme and offered to anover eny questions when the eervice was aver. Twenty minutes were alloted to the aubjecte for complotion of the attitude acale. The aervice we concluded in the usul manner and the experimenter enowered eny questions that cens up.

\section{Ane and Educhtenn Lomin}

For the purpeses of analyzing the date of the etudy the eubjecte were divided Into three age groupe and four education groupe, thue giving a metrix of twelve celle. The age groupe werel (1) wbjecte 
between 15-30 yeare of age, (2) eubjecte betwen 31-50 yeare, (3) oubjecte 51 yeare of ege or older. Thio divielon wes beed upon eeveral coneiderations. The eubjecte in the piret ege group heve lived noet of thelx Llvee in changing Cathollc Church. It lo expected that they w111 be much wore accepting of change than the other groups alnce change Is lese unusual to them. The subjecte in the escond group have apent more of thels livee in o Church that did little changlng and hence are expected to be lase accepting of change. The third group will have epent much wore tiwe then elther of the other groupe in an unchenging religloue atmosphere. It 1 expected that they w111 be the not coneervative group.

The divieion of aducetion Into four levele seemed the woet practicel from the standpoint of the general population to be eurveyed. Grade sohool, high sohool, some eollege, and a completed four yeer college eduoation were conaldered the easlest divielone to make in our population alnce they ake underetandable divialone for the reopendente and each level constitutes an appreciable educational difference. Yeare of echool completed thus conetitutes our operetional definition of education.

\section{PREDICTIONS}

In the light of the previous diecuesion and the reoulte of the pllot atudies the pollowing predictione were indes

1. Scoses on the present halp of the ecele will be higher than sooras on the future half. This prediction wa teated aith a teet for correleted date.

2. Younger Cathollce will tend to be more libaral or accepting 


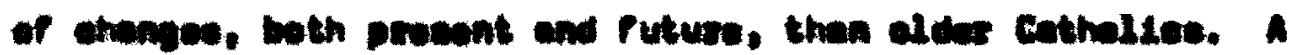
eerioletion wa computod te teat this hypotheule.

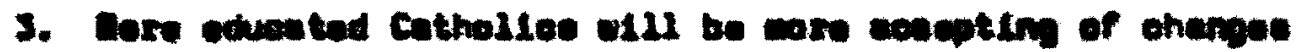

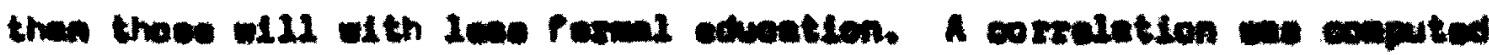
te toot chis hypotherie. 


\section{ounpran III}

\section{nouts}

\section{I. cturen mats}

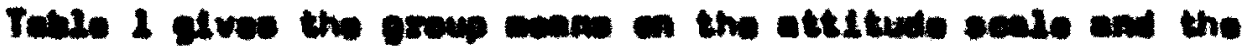

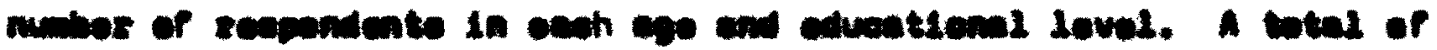

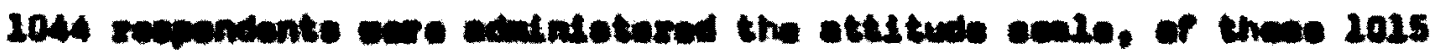

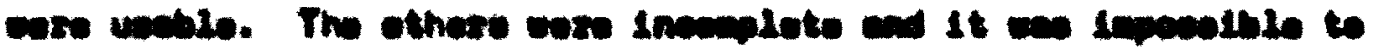

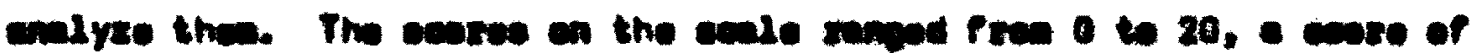

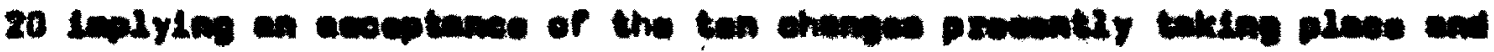
the ten hypthatred ohenoes.

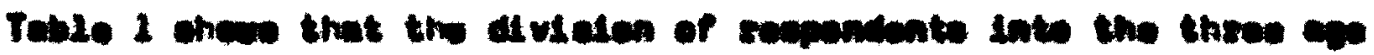

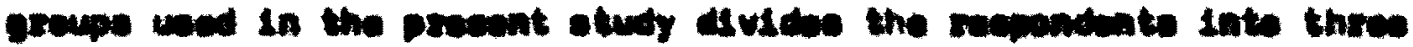

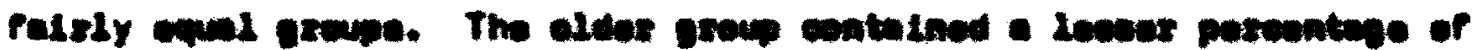

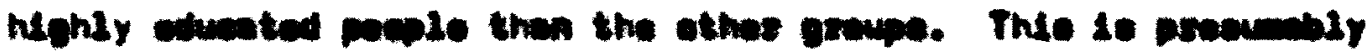

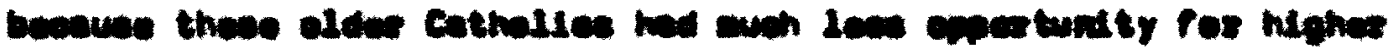

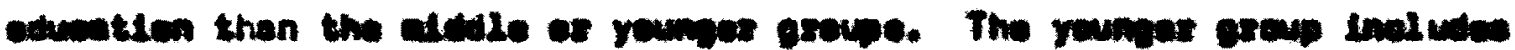

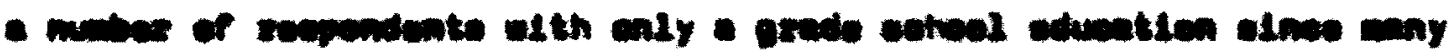

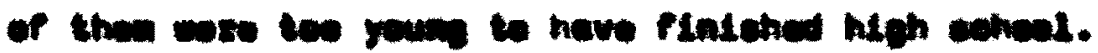

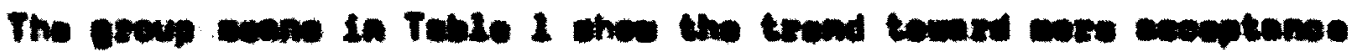

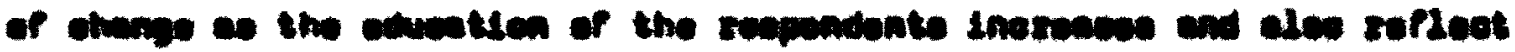

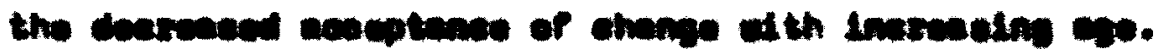


Taus 8

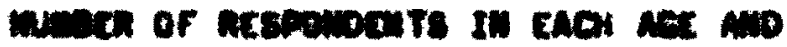

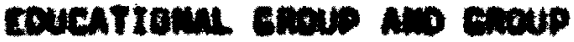

ncall on me atrituor

scule

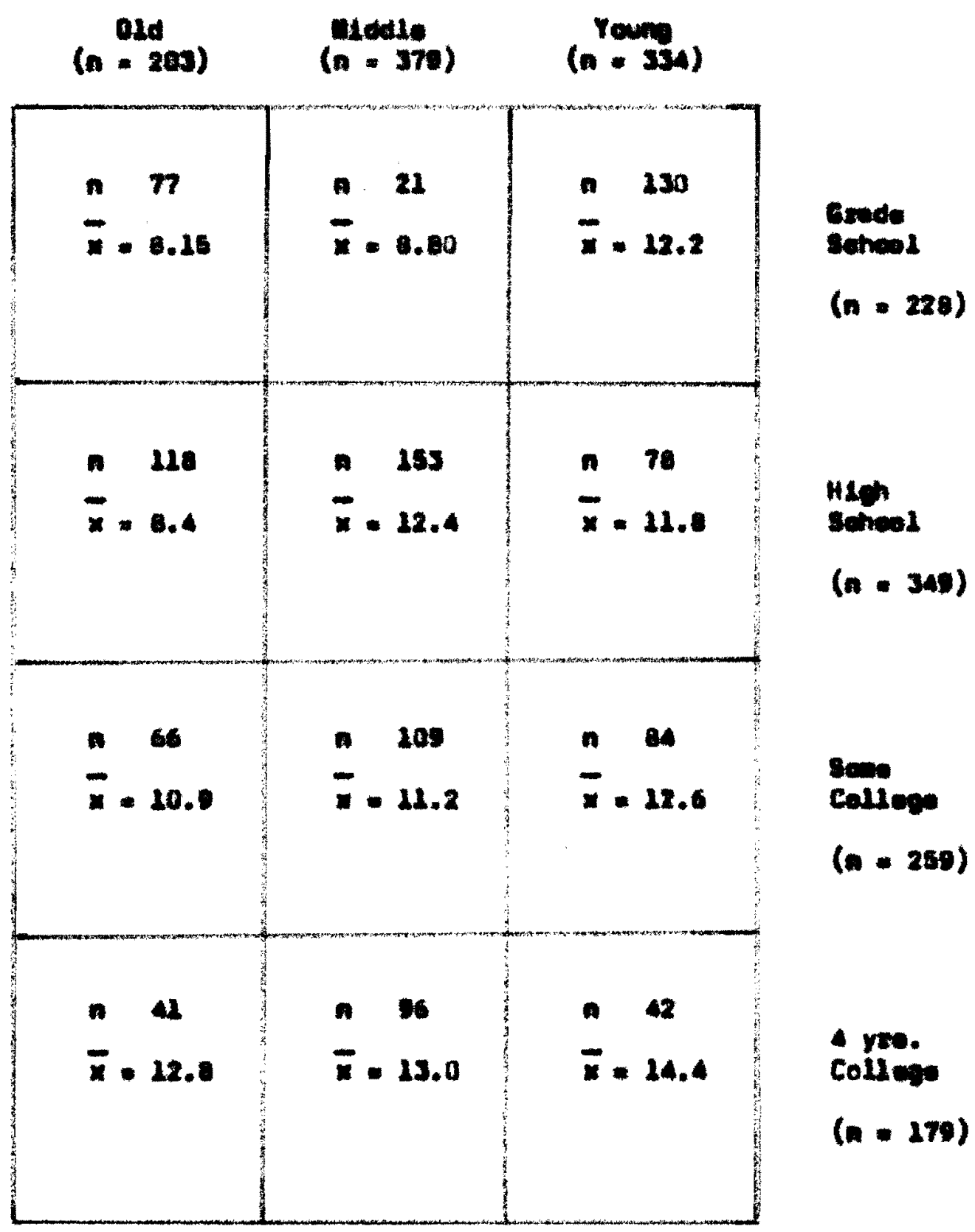


In fig. I the man scoras of anch educetional graip ere plotted gatingt igs. Lower scorwa ise indic thun of coneervation and higher

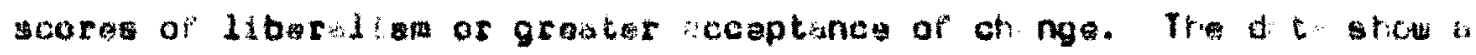

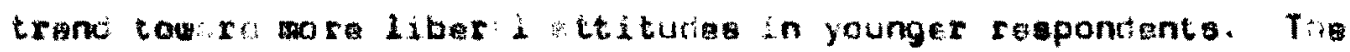

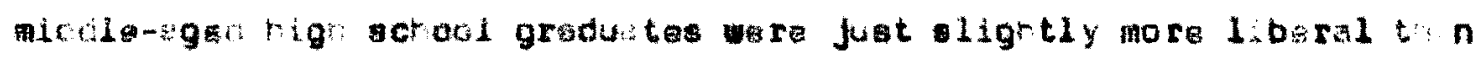
the jounger igl school grertutus. Tho corrat tion botasen scores on

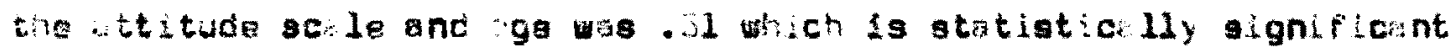
the. If lovel. Tre correlution between acores on the attitude scale and oge with efucetion bertialled out wo .36. Thus younger padple se aeen to bo gignificantly more acospting of change then oldar peopia.

In Fig. 2 tha mean seores of ech aga group are plottad ageinot the four aductional levels. The trand toward mora liberal attitudie wit incroaste nucation is soen in theropt. Tra corralation between

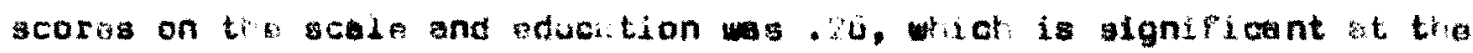

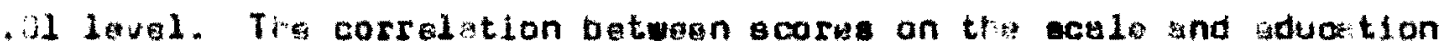
wit: aga prtialled out wes .35. Thus aducation 1 s sean to be significantiy raletad to accoptanea of otango, nore aduo ted respondents tending to accopt otangas more radily trim le foducated onas.

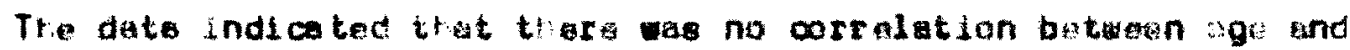
oducation ( $x=-.05$ ), implying no overleo in the varianco accountac fer by the two varlabloe, 30 multiple $f$ computed. The multiple botween scores on the scale and zge and education was .41. About 16io of the varlance on trese ecores is thus predictoble from age and educistion.

A t tost, using the formula for corralatad observations, was done on the deta fron the future and present nalves of the scale. The 


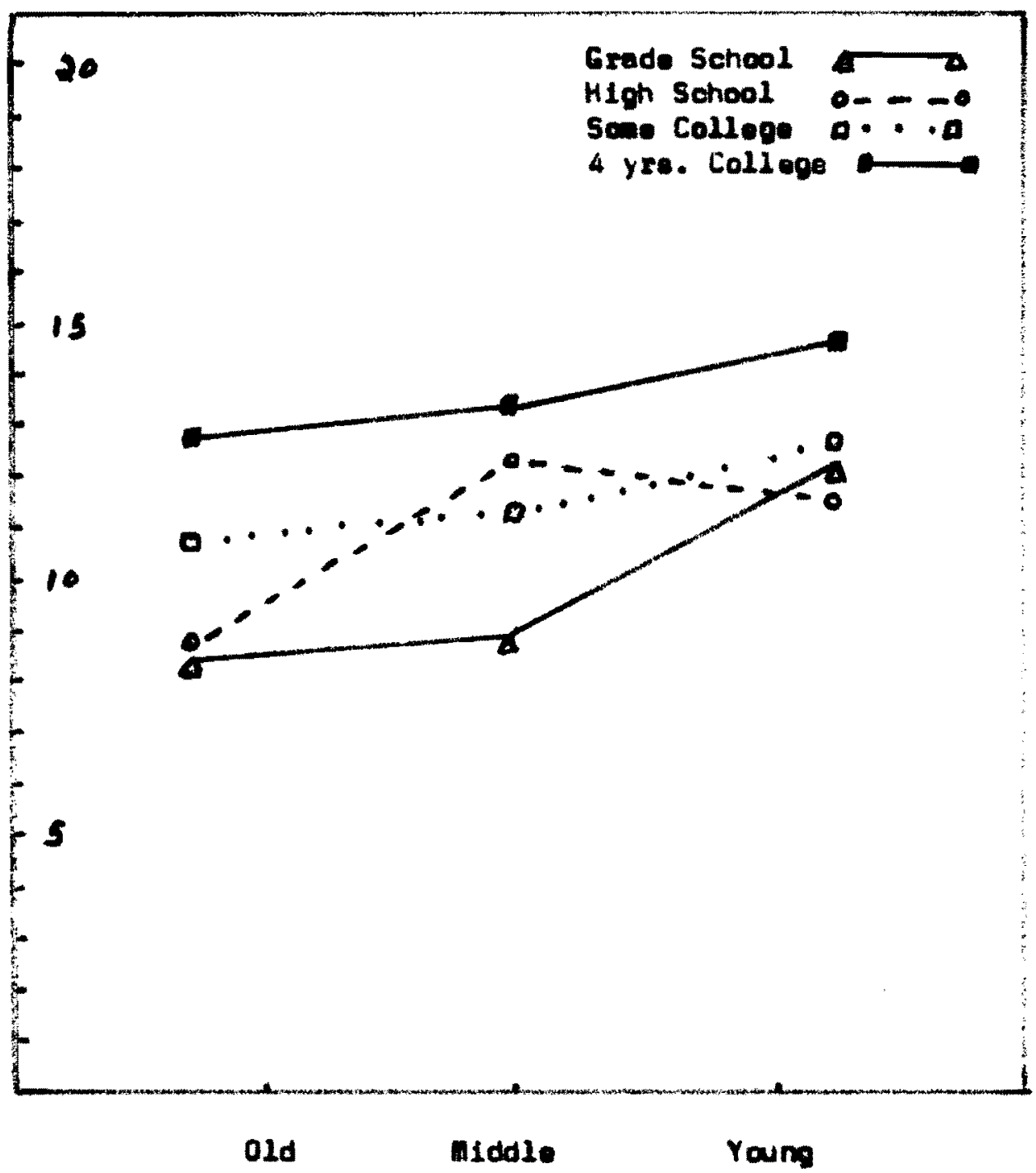

AGE

Finure 1. Dean ecores on the attitude scele of the four oducational levele plotted againot ago. The higher the ecores on the ecalo, the more liberal or accepting of chenge ere the ruspondents. 


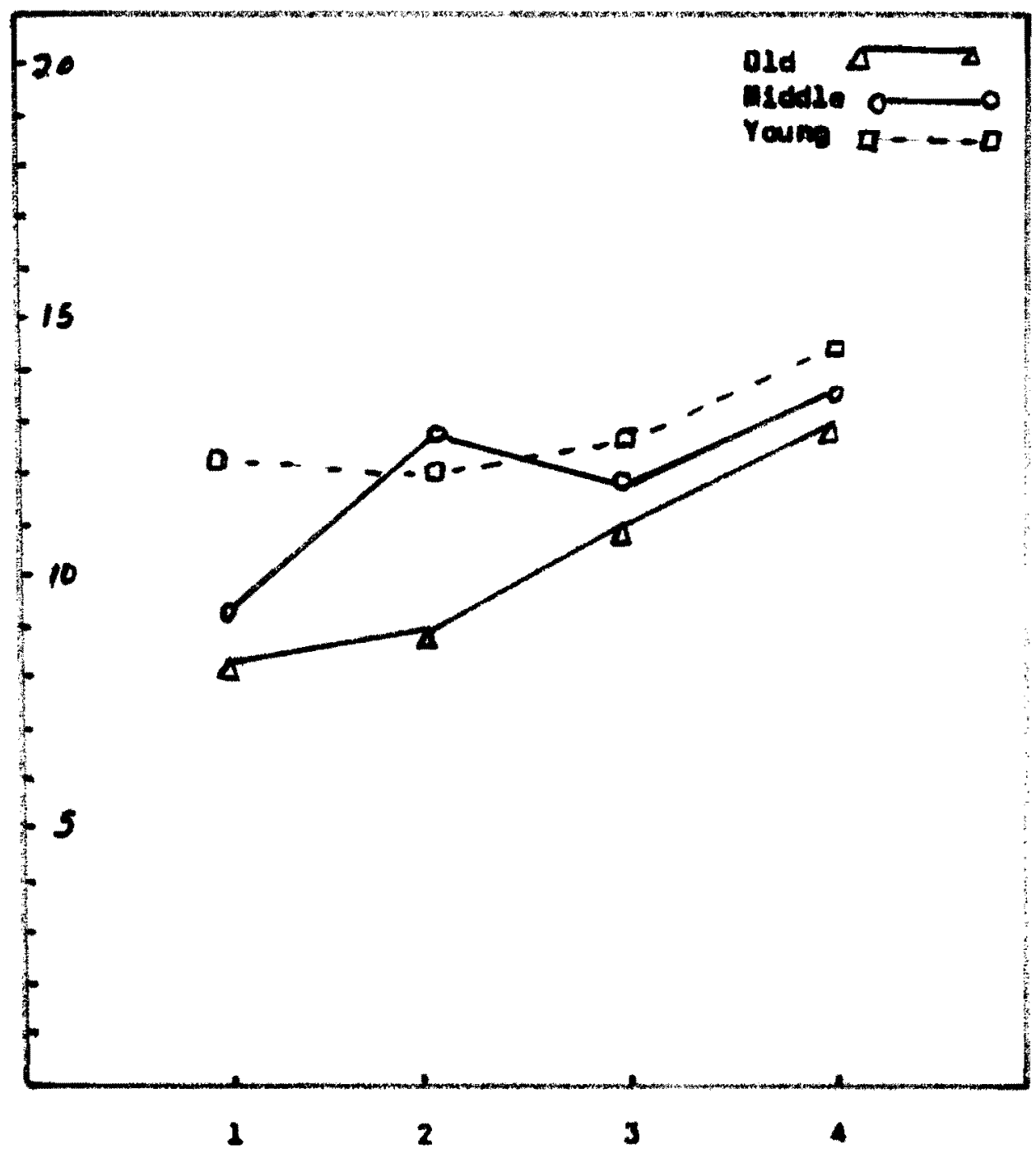

EOucationnL LeVELS

Eleurx 2. Dean coores on the sttitude ceale of the three

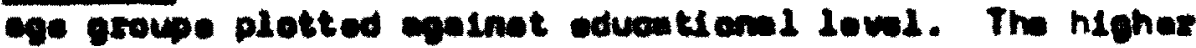
the ecores on the ecule, the wore liborel or eceopting of chenge are the reopondente. 
t - obe. we 36.51 (p. <.01) Indsceting euppert for the hypotheale thet Cothollce ere are accepting of chenges el ready inotituted than they are of future hypotheelzed chenges.

\section{Sout InoIvtount ITtens}

Sone of the 1teme in the attitude ecele are of opeodel interest beceuse they repleot nttitudme on eattere of great importanon in tho Catrollo Churoh todey. Itwe 6, 11, 13, end 16 have bem annlyzed wore fully than the othere and ere preaented in the rollowing rour pigurwe to show the reults in grester data11. Itese 6, 11 , and is wre from the future part of the soale and 1 tem 16 lo from the present part of the ecole.

$\operatorname{1+n} \underline{6}$

Flpure 3 showe the perountage of each group egrealng at th 1 tea

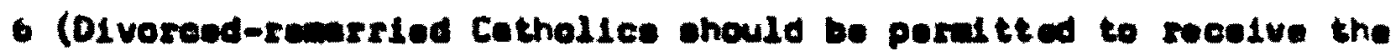
escrmente, especielly Comunion, wen though their eoophd earriege 10 Invalid eccording to noxnel atandarda). (uverell, $61 \%$ of the reapondente apread that pople Involvad in invelid erriegee should be ellowed to reosive the eacreiente. Floure I whow that in ecen group at leest bof of the respondente chere thle poradeolve attitude. In the youmer and core aduented proupe almot $75 \%$ of the reopondente exprese dinagreament alth present policy. Soventy-four peroent of the

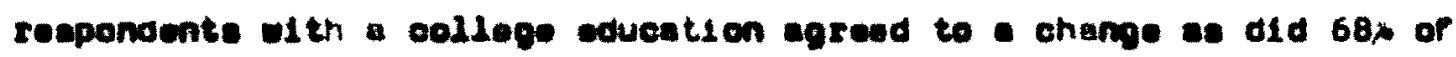
the reepondente under thirty yeers of age. 


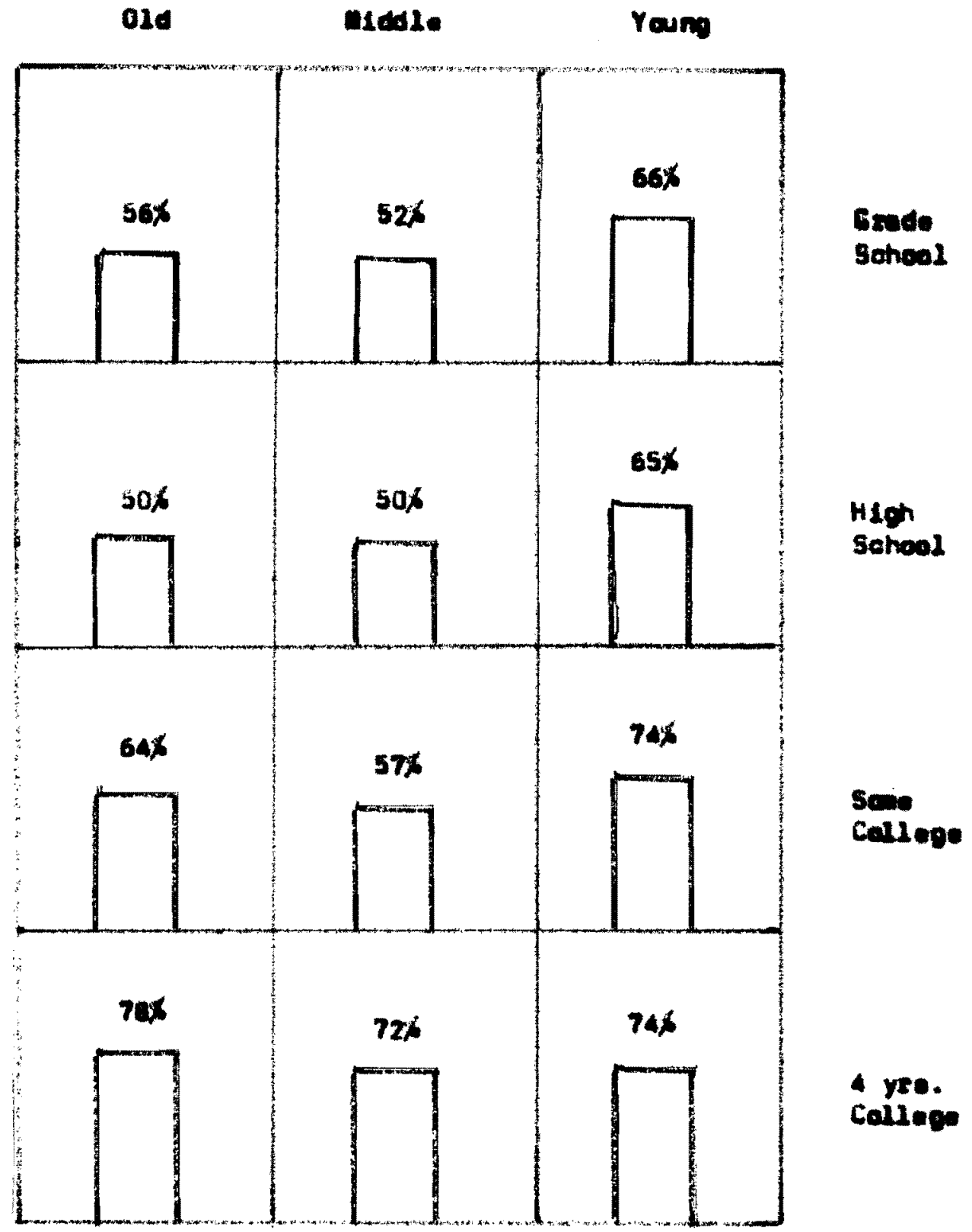

Elourns. The poxomtege in sech oroup egrosing

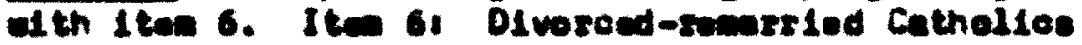
chould be potwltted to seoulve the eecremente (ocpaledly comution) aven though thols cocond marlepe it invalid cooording to nomal etendarde. 
Itron 1

flouse 4 pive the reoulte of the ewrvy for Item 11 (Prieste

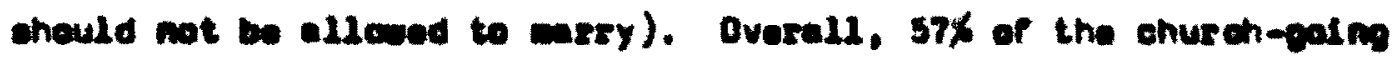
Cothalies in this otudy indiente that they disepres alth the catholie

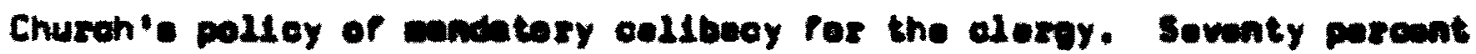
of the onuroh-gere with a completed oulloge eduostich diengrond of th prosent paliey. S1xty-elx peroent of the younger seopondente deegreed alth prowant pollay. Ameng the younger Cathallos wth eodlege

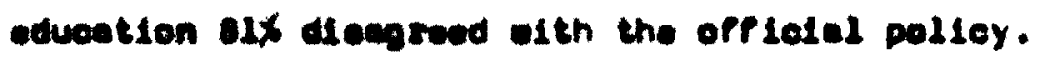

\section{Itra 13}

Figure 5 give the pereanteges in esoh group al epreating with

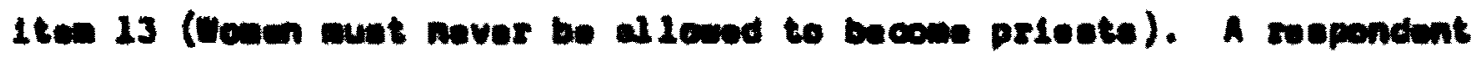

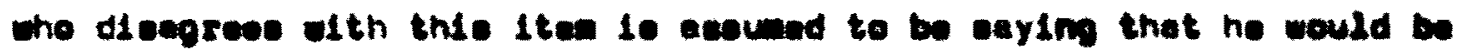
In fever of having remile clergy in the Cothosle Chureh. elnoe to epreo

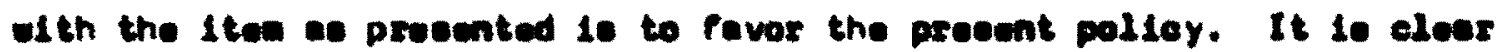
prow the dete thet the vet ejexity of our reopondente aso unpreperes to ecopt women prieete of thie t1ne. Owerall, 16\% lleagreed with the prohlbition of Pemie aleroy. Thirty-two poroent of the respendente

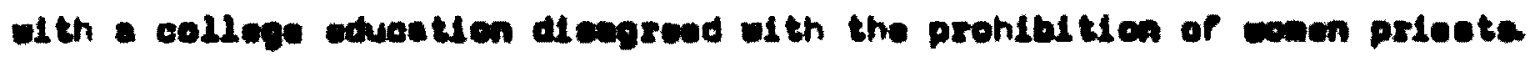

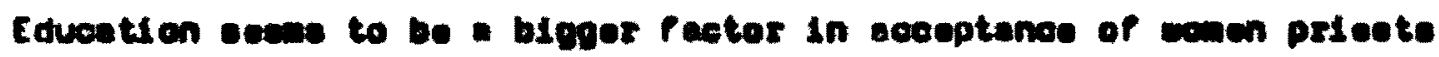
then age.

$\operatorname{Ltes} 16$

Figure 6 gives the rewite of the wrwy on Iten 16 (To be a god

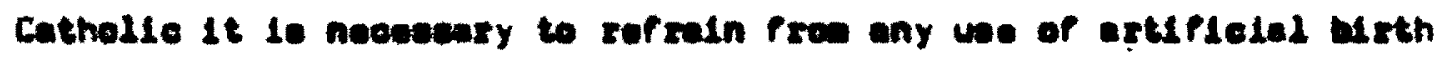
eontral dovlees and the brth contrel pil1). The cute ehes thet 


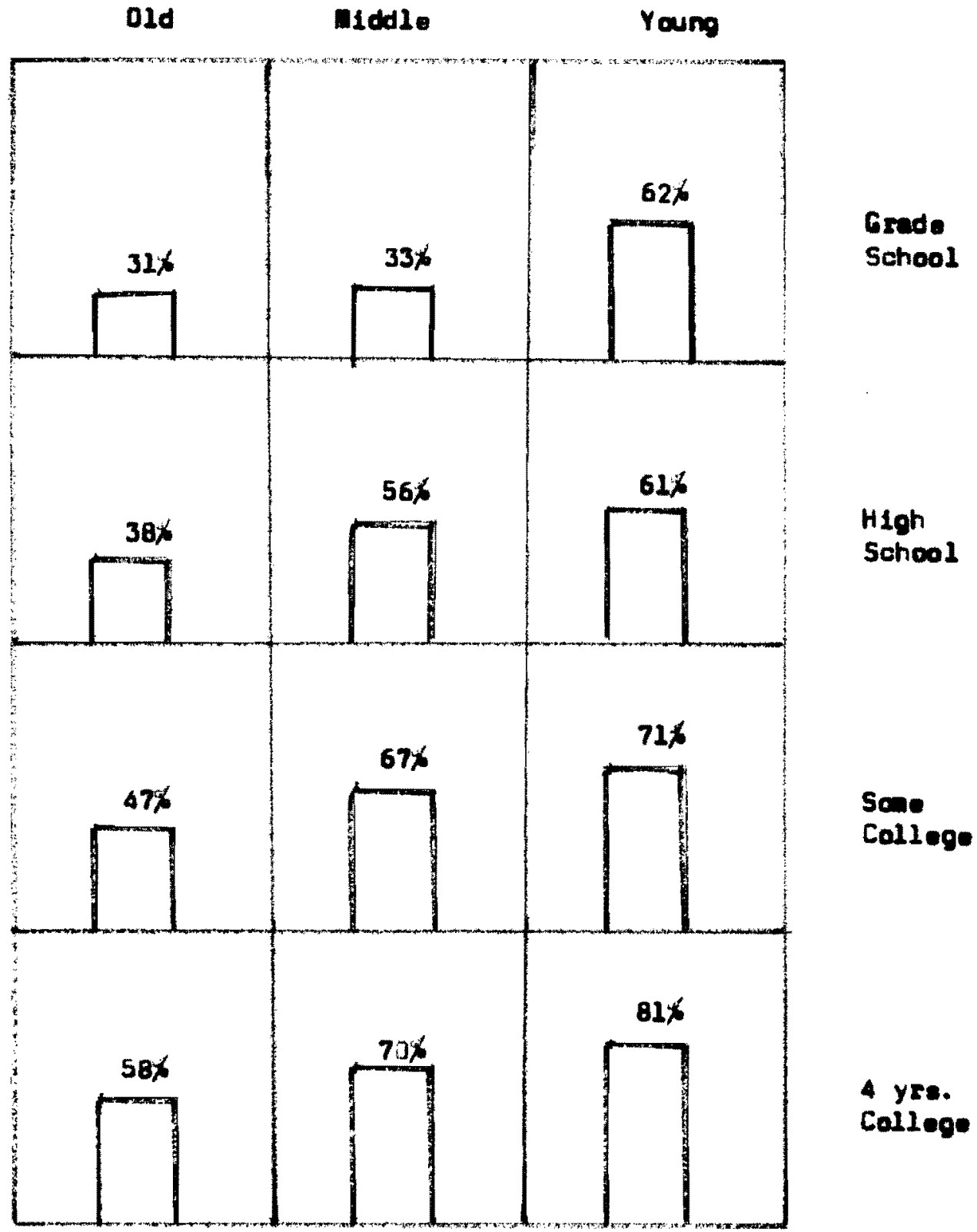

Eloure a. The percentege in each group disegreaing ath Iten 11. Iten 11, Prieste ehould not be alloeed to anry. 


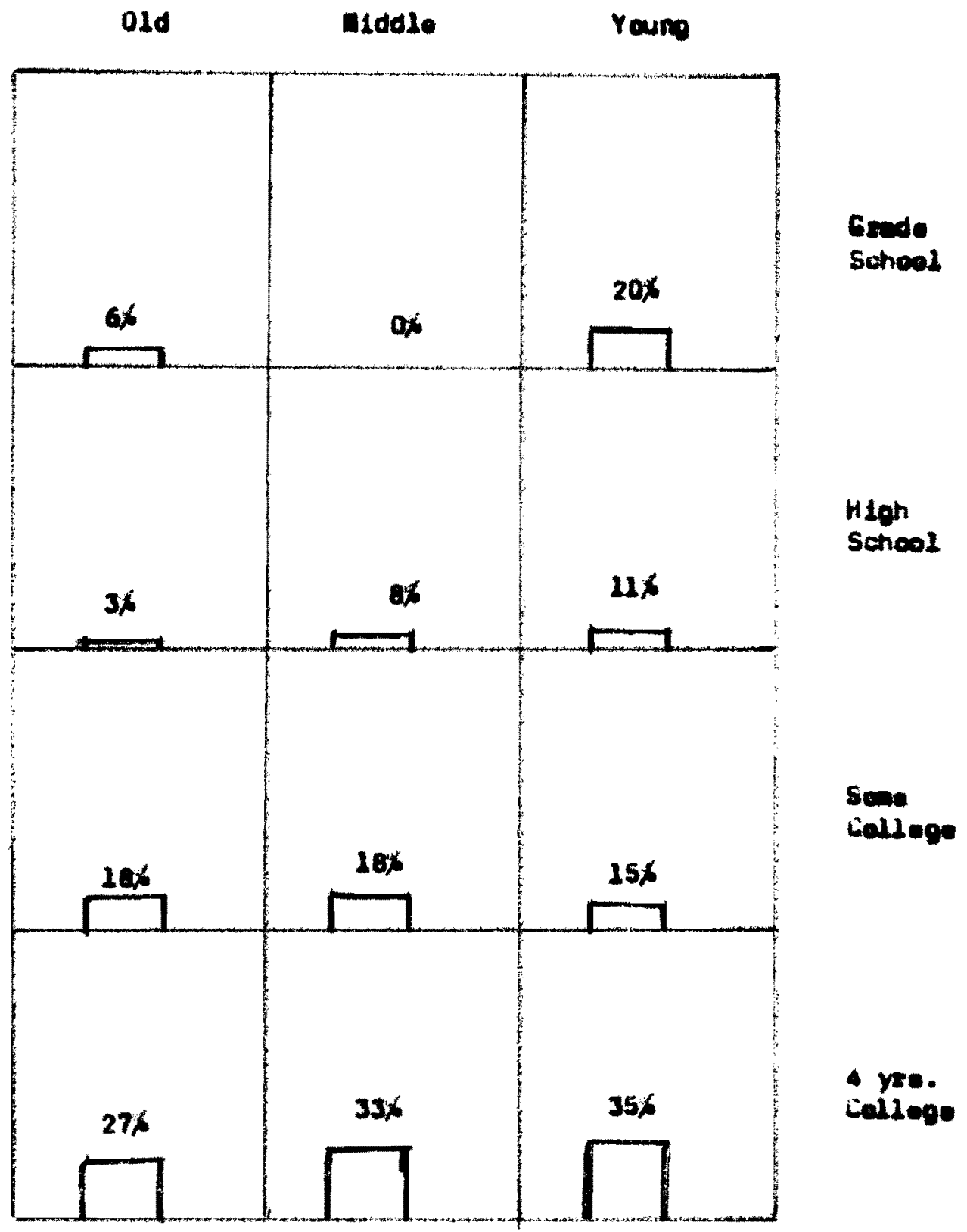

Eloure 5. The porcentege in ench group di eagreeding

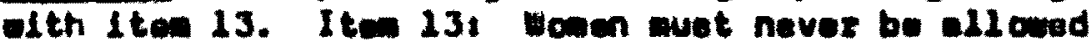
to bacome peleote. 


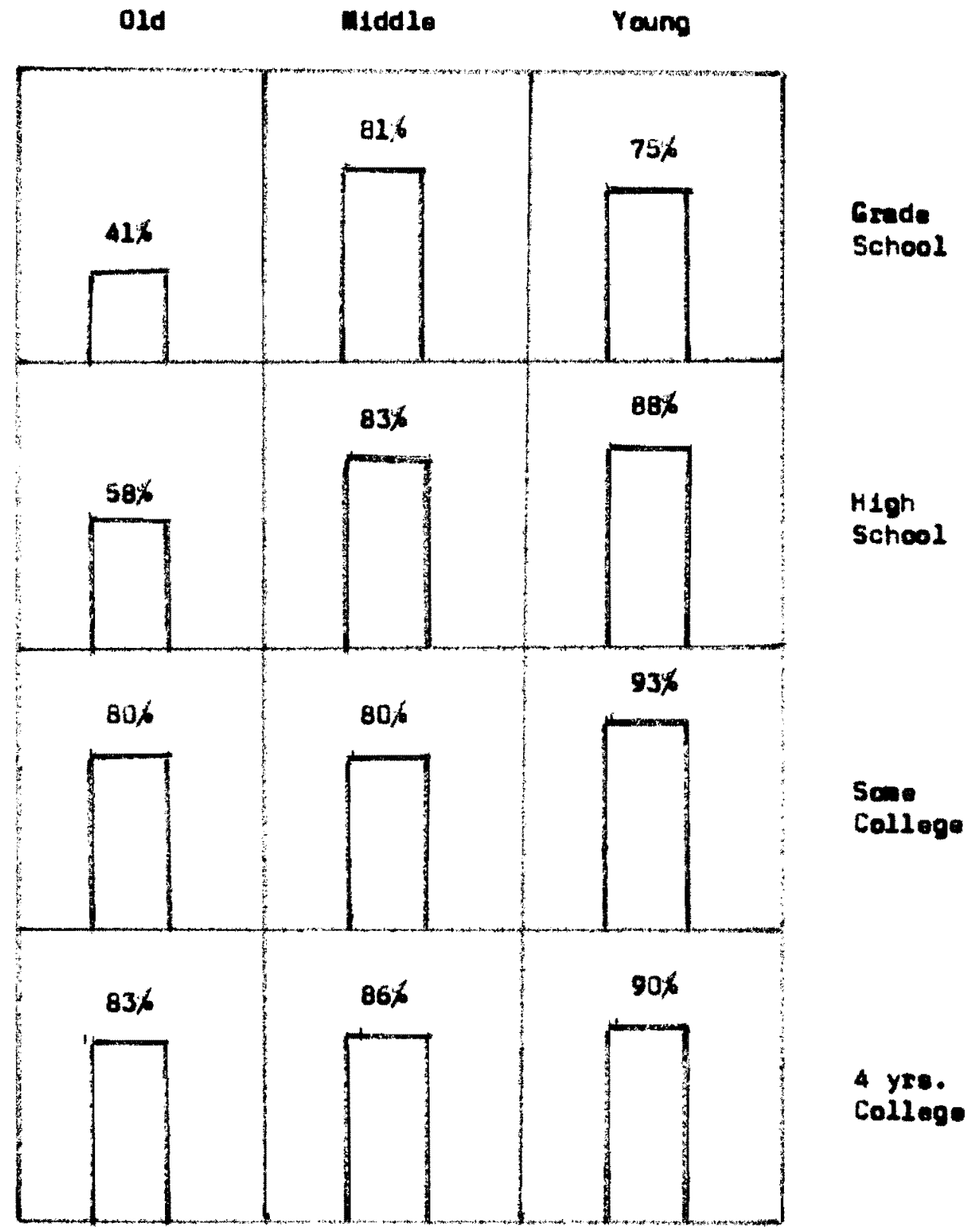

Floure 6. The percentage in ach group diesgreaing ith Item 16. Item 16: To be a good Catholic it 1. nocesenzy to refrein from any uee of ertiflciel birth control devices and the birth control pill. 
overell $77 x$ of the respondente do not wecept thle lten. Amang younger and hlohly eduated Cethollos only a eall peremtage ecept the offlelal poeltion of the Chureh forbldding ertiplolal bith control.

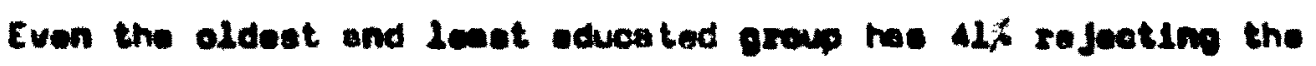
troultional teaching of the Chutoh. Elghty-ewen poteont of the reopondonte of th a edlege cduontion do not acoept the Chureh's poestion. 
CHAPTER IV

DISCUSSION AND ImpLICATIONS

1. cenERAL DIgCussion

Ovar 97, of the reepondente (1.35 out of 2J44) raturned queationnises that earo comploted In overy detali. The low parcentege of Incowplote returne showe trat church-golng Cathalica arn not adverse to raporting thels viawa on the ftems of tha ecala. Soveral of the quastiomalree conts lned rewarke by the raspondente indlceting tholr approvnl of the survey. No agnificant nogetive ronarks aer found In the dete. Thase reaulte Indicate thet religlous ettituda research under eenditione of eccleslectical approvel and durling churct ecruloeo alght be very sucecesful onong cathollce and even other rollglous proupe. At letst the Cotholles in thlo study vere very cooperative and Intarested in the roserch.

The ralevencs of the lteme and the ealiencs of the roligloue eteophere soan to be conditione that unabled the reoseroher to obtain sore complete reoulte thon ueundly poesible in wolel poyohology

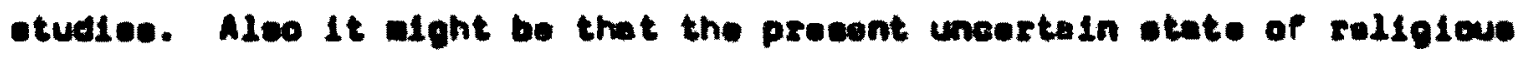
dieclpline and doge Ineline people to be ware Intereeted in both expreselng thels ow viowe and learning the viewe of thels fellew. Detwe (1965) hav eontended that it is poosible to detinguioh botcoen canservetlve and libaral sttltudes towerd aurront ldoes and the exleting Institutione and cuetcoe of any indlvidual rellolous group. 


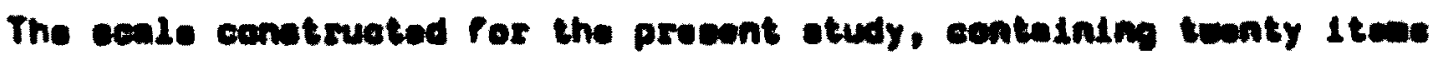

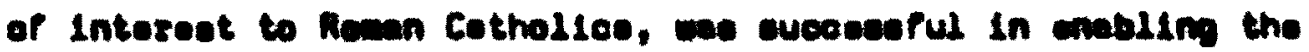

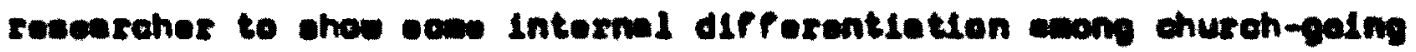
Catholdce. The fact that soores renped from 0 to $20 \mathrm{en}$ the eode

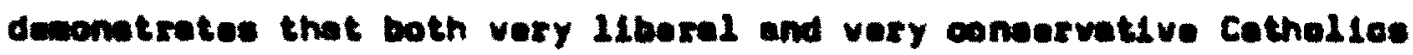
ot111 werohlp together.

The carrelation betwen ceores en the attitude coale and age wae .31. Younger poople ere elgnifieantly were ecepting of change then are older poople. These resulte indieate that roliplous ottitudes

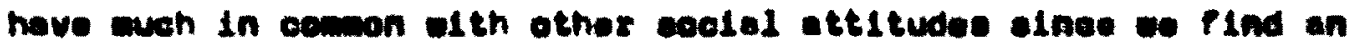
Ineresolon conoervative trand with inoreating age.

The correlotion boteren coores on the attitude son le and advantion

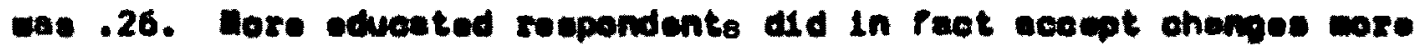

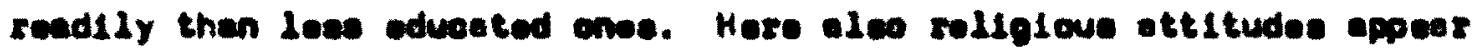

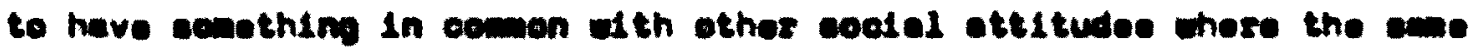
phenemonon eceure.

Prosery' (2966) etetement that noet otudies of attitudes chav

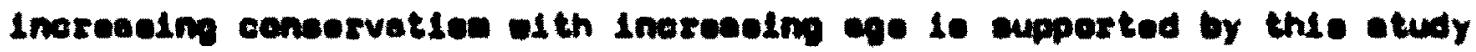
of rollgtous attltudes. Stark (1965) found that wre oducation bas

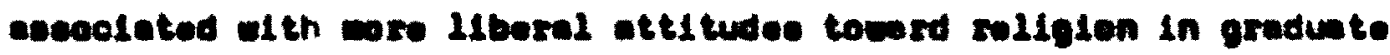

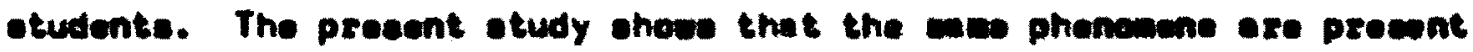
In a population that oevore a grenter oduoatienol range.

The 1 teat dene en the date frem the "ruture" and "present" halves of the eeale Indicated eupport for the hypothoule that Cothalles are core eocepting of changee elreody ande than they are of hypetheelzed changes. 


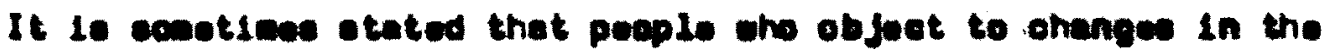
Catholie Church eleply lave the Church. The present etudy of fore no

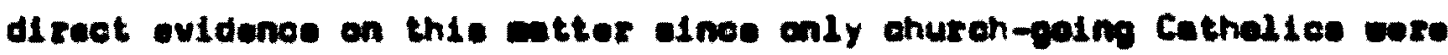

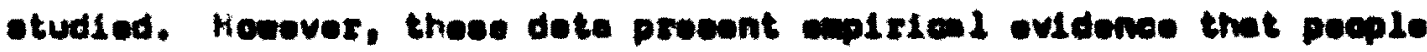

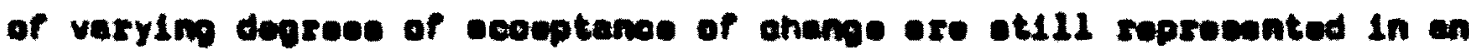
ordinary sundey cengregetion.

The three prodletions ande in boelming thlo etudy oore eupposted by the dete. Church-polng Catholles ases to be aro asopting of ohanges elreedy ade. The etudy offere no dreat ovidenee of ohy this 10 wo, although it alght be souecouble to hypothedas thet fandierity Ith the ohanges and reepest for cutherity ere Invelved here.

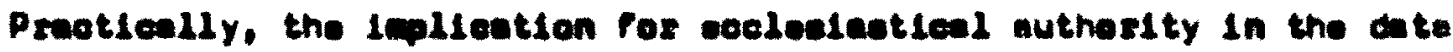
I. thet churah-goere do scopte ohanges are readily owee thoy ere Inotituted. The date Inply that sene of the eentroverey that if

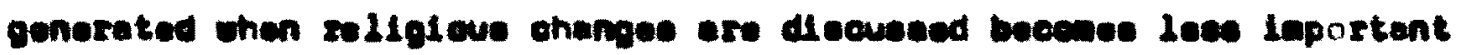
onev the ohanges have boon eade and people bogln to live wth thea.

The predietion thet yourger poople would weopt ehange wore rowdily than older pople was we eupported, the corralation betwem

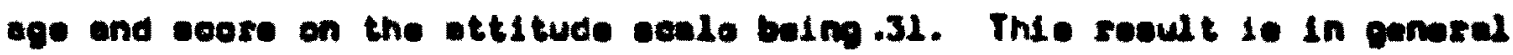
agreament with other etudise of eoclal ettituces. Wo one ean do woh

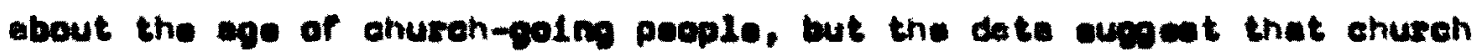

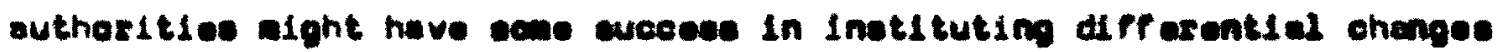
for the various uge proupe. Older ohureh ambore alght be wore content to live out their live eccerding to traditianal nome ohile youngas

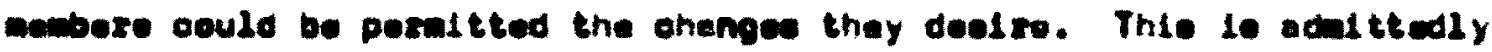

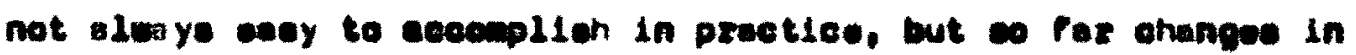


Catholic dife heve aleye boun wede for all Catholles and the peosibility of changling thime for verioue age eroupe has not beon atcopted.

The predietion that core odueation wa related to greater aceoptence of change aleo we supported by the reoulte. The eorreletien

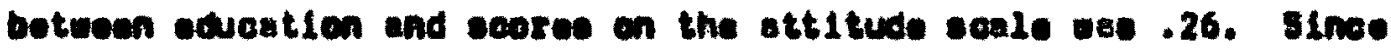
educetion hes alwaye been encouraged by the Cothelle Chureh in the United States and can be further developed, our date alght be eald to eupport the velue of the edwetlonal endevver if religloue authoritioe

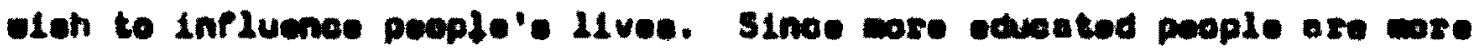
aceopting of change we ught oxpect thet in a ocmplew pluxelletio

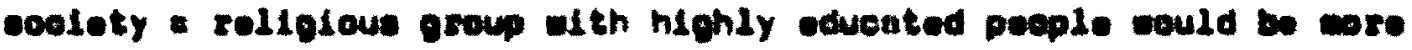
edapteble then a group of loas adueated pople.

\section{Discuss Ion or sone Inoruroun Itrens}

\section{$\operatorname{In} 6$}

Iten 6 (Dlvaread-rumerlod Catholies ohould be perielted to revelve the aecremente, eapecially Cemunlon, oven though thals oveond merriege to invalid oeeording to norml etanderde) tape vary Importont area of rellglou thought elnoe lt rofore to enrriege, ceneldered a eacranent or sacred alea by Aoman Catholice. Figure 3 glvee the peroentages of Cathelies in each age and edwational preup tho egresd

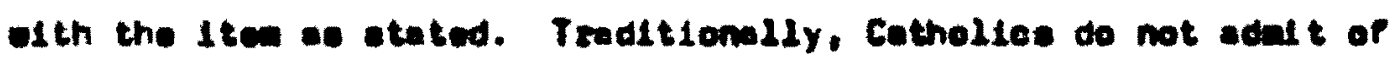
second arriagea unlese one of the epouses in a plret earriges ha dicd. However, today woot Amorioen Catholle fanill os hive oeme relative os

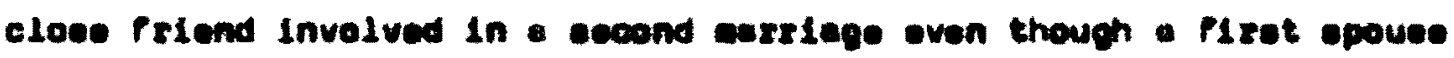
is Living. Acoording to Catholle theologleul prinalples this is 
cenoldered an invelid earriege. The ponalty for entering the encond

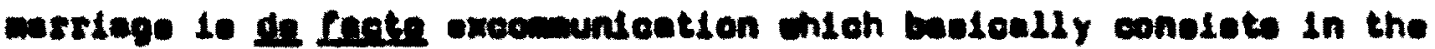

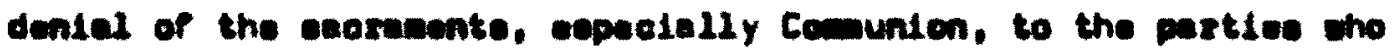
enter theee invalld merlagee.

The chureh-galng Cotholies in the proemt otudy ecen to Indiento that they would like to ees a polioy in this meter. Overnil, 61\%

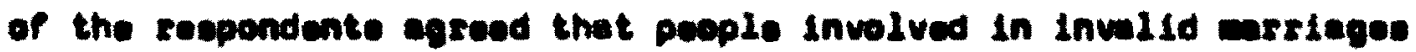
chould be aldowed te rooolve eaeremente. Figure 3 shew that in each

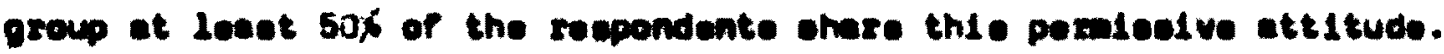
In the younger end exe eduented proupe dwoet throe-guertere of the reependente dieagree wlth the present palloy.

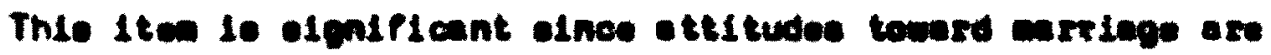

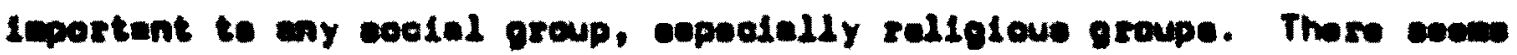
to be a tolerenoe of dovletion pres the religlous noxe eot up by Chureh

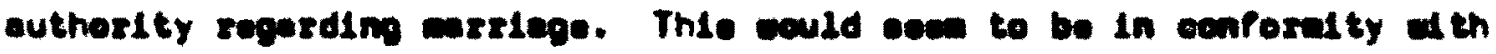
the wore lenient and telerant vies taken of ewoond weriegee by the

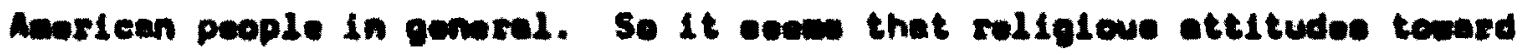

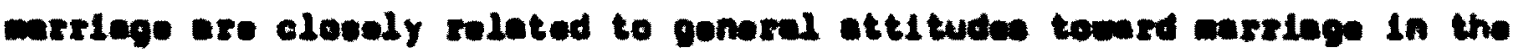
overell culture.

Ae a etter of present polley ell Cethollos lmolved in a coend

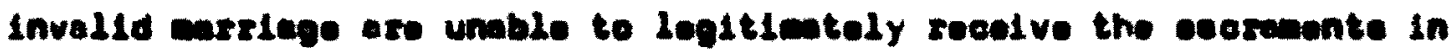

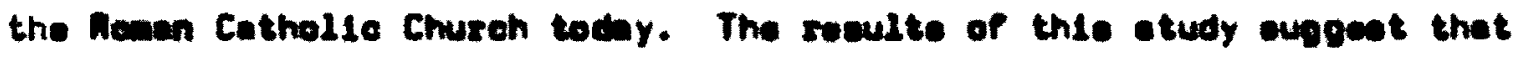
any praetielm Cetholles ere not in egrwoment wth thie polloy. Ao a

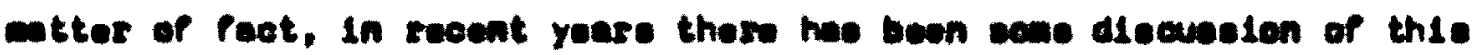
problem in the Chureh, but no ohange in polley heo over bean eade. Our

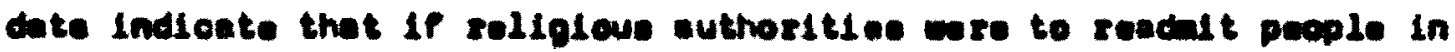


eccond exrleges to the eacremente they vould pind conelderable eupport anong church-goling Catholice for thle radical change.

\section{Iten 11}

Flgure 4 glvee the reoulte of the eurvey for Item 2 (Prieete chould not be allowed to marry). Ovarald, $57 x$ of the respondente Indicate that they dieegres at th the present polify of eandetery cellbacy for the clergy. Anong the younger Catholles oith o college oducution 8lim diegreo with the of flelel pollcy. This lo anter of some leportence eince the clergy lo recrulted faen anong the ranks of younger educeted Catholice. The etudy Indiates that the prosent ohortage of Catholle olergy wight be due to the Church's Inaietenco upon cellbacy ae a condition of the prieethow. It 1 e comon knoulader In eoclal ectenes that people are very muen Influenced by the vi we of thel: poere, end it lo elear thet young oducated Catholloe de not eupport mandatory cellbecy for the elergy. older and leee educeted Cotholles ere weh lese inclined to eceot a mercled clergy. Thio io clear Prom the percentages in Fig. 4. Thore ere probubly eeverel reseone for thie, but it eseme that catholion have been very conecloue of the unmeried etatue of that clemgy and have been proud of this fect. If andetory cellbecy 1s not ineleted upen in the puture it alght

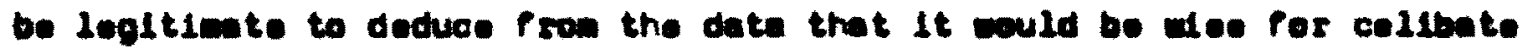

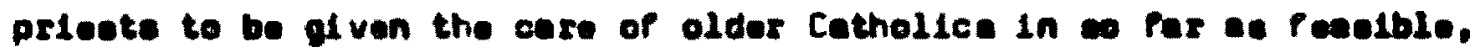
and eerried elergy eesigned to the cere of younger peeple.

The attitudee of Catholice towerd elerical collboy are probably aleo related to other social ettitudes and velues. In countries like Hollend, Cenode, and the United state ghere the queetion of clexion 
oulibecy hes been under eritiod exedination fos come time, there are

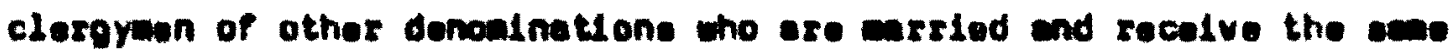
etetue and ecceptenoe as Rom Cetholic prieste. In thees countrieo the leaue of cellbecy is much more cruciel then in treditionaly Catholic countrios Ilke Irelend, Spain, and Itely. The present dete offer no direot ovidonee, but wa aloht hypothudze that Catholice remald that: rellglove ettitudes in eccordance ol th thatr Ilving exporionoes. It would be intereoting to emple a oroup of Catholice IIving in areas where many rellglons Interningle and enother group in erees in which Cathollee had practically no centact olth other alergyman. If we found atonlficant differences it aght provide some evidence for the hypotheals.

\section{$\operatorname{Ltan} 13$}

Figure s given the peremteges in woh group dleagreeing of th Item 13 (Vomen wut nover be allewed to beoome priecte). Diesgresing aith thie 1 tem inplies that the respondent could be alling to aceopt - fanale clergy. The dete Indicete that our reopondente ere for the eoet part unprepered to eocept wowen prieste ot the preeunt ues. Overall, only $16 \%$ d angread with the prohibition of Pemie elerey. riguse s showe that only anong the higheat oduceted Cathollee eould there be even a minded ecceptence of female clergy. Aoseptance ranges Prow of In one oroup to $35 \%$ in the youngeet and noet highly educeted group. However. evon this exount of ecoeptanoe lo rether aurprielng when one cenoldere the fect thet loes than ten yoero opo no one had even

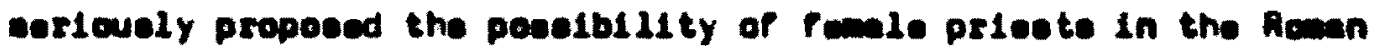
Catholle Church. 
The date of the etudy auport the Churoh autherities' contention that of present tatholic pople are unulling to hove fande clexgy. At the eve tive the percentege of these whe exe younger and ware

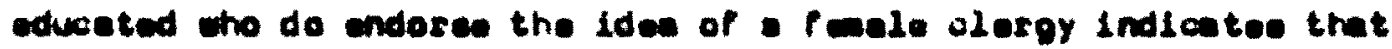
porhepe the time will oome when the Ides w111 heve to be taken were enrlously. Sinse it cppeare thet religious attitudes are related to other sooled ettitudes, It wght be eduleable for churoh cuthordtion to follow the forturne of the women'e liberation novement for clues to the future. It eould be intereeting to take the preoont 1 te and reodinifetex it in five yeare to ses if the number of people who ecoupt - femede clergy inorenese.

\section{It) 16}

Floure 6 gives the resulte of the eurvey on 1 tem 16 (Te be a geod

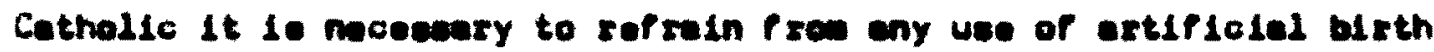
centrol dovices and the bith control pl11). The date hare ere vory apnifloent. The offlelel Cetholic pooltion es expounded by the Pape and bienepo of the Catholic Church io that ertifisiel urth control of eny kind 10 imorel. The date whew that $7 / 6$ of the reapendente do not ecoept thle. Among younger and wore oduceted Cothollee coly e very enall percontage (10\% or loas) eccept the offlalel pootiten. Even the oldeet and leaet eduoated group the 4 lx rejeoting the treditional teaching. This ltew wo Included in the presant half of the soode

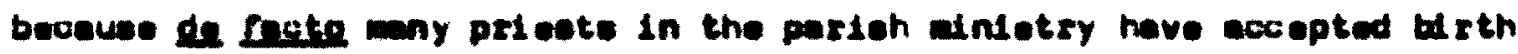

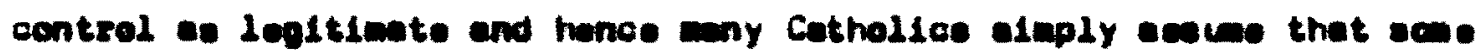
change in Eatholic tevohing he ocourred. It aight be ergued that ance the highent athoritles have not glven eny epprovel to o ohenge in 
Church tecohing that the Item belonge on the future half of the eode. This etudy inoluded it a pert of the present coale and it appose as If thie judgent hee been valideted by the date.

Thie Item egein offere coms emplricel support to the contention that rollgioue attitudes ere releted to other oocial ettitudes. Sexul nore have undergone a not-teo-quiet rowolution in Amerion eociety and the reopondente' attitudes toward birth control reflect this revalution.

The fect that oo any of the reopondente dieegrea with offloial pollcy onlle etill attending eorviceo regulerly eloo eupoeote that man Cathollo ohurch-oose eccept the teaching euthority of the Pope and blohope but only to a linlted degree. Our date offer no direot evidence on this watter, but they oupgest that a otudy of how Cathalice viow authority would be intereating at the precent time in the Cathalio Church.

w. 6. Plppert (1971) reporte that a soclologloul etudy ande by the National Opinion Research Center notes that "there esene to be 11ttle reason to doubt that apport emong the clergy for the Church 'e teaching on birth control and divorce is waning." The report aleo otatee that eome Catholic prieete ere belns eupported in their denende to waxry by thelr perishonere. The dete of this present etudy of ohuroh golng Catholice show some light on theae reporte. If the etudy is accurete, future evente should eupport the date and concluelene that heve beon drawn frou them, elince, after all, the eoolal ecientiet contende that poople's actiono hove eowe relation to tholr ett1tudea. 


\section{Funthen mestaon posgrerLitIEs}

The proemt otudy the bean a oorcietsemel one. Out the reculte

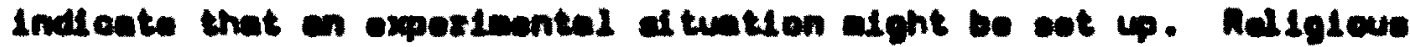

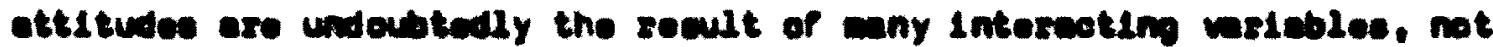
ul of Antoh ore oentrolloble. Out the proount etudy indionted that

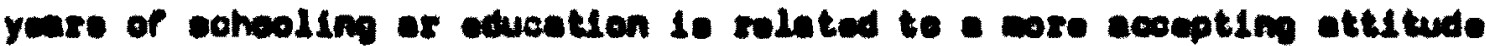

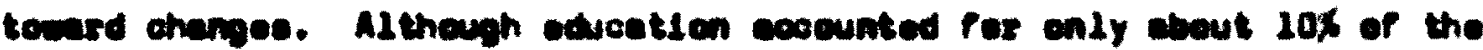
varlenos in our woores, this is enough to Justify purther attempte to

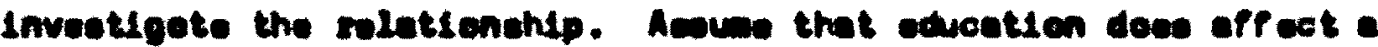

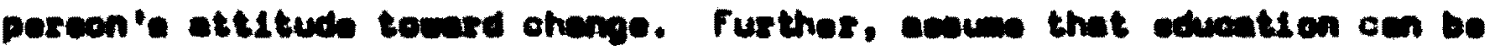

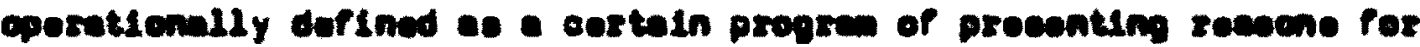

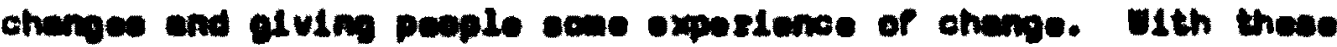

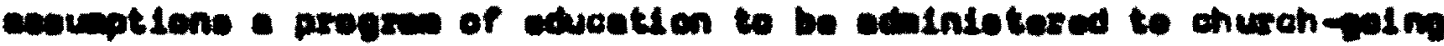
Catholles oould be deatened.

The plret etop would be to seok the noosangy pordiestere to undertike the expesinant. The pindinge of the preeent etudy sheuld

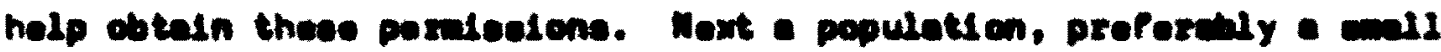
dlooses, would be chesen and a rendom eample of elght perlenes enleoted

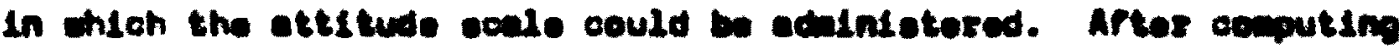

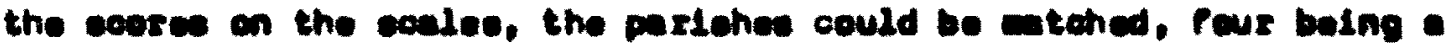
ocntral and fous an exporinental geup. The oxperinented group could

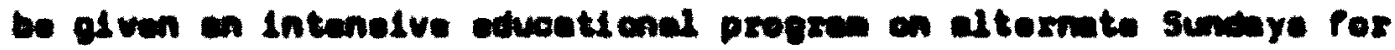
el $x$ wonthe, Pellewing enioh both groupe could be readainiotered the ettltude eoede and dipf errenoes noted.

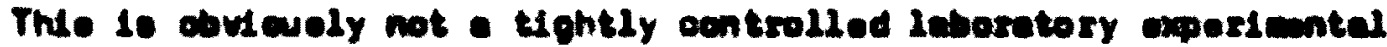
etudy, wi it sowe that it could bo althin both preotioel end othioal 


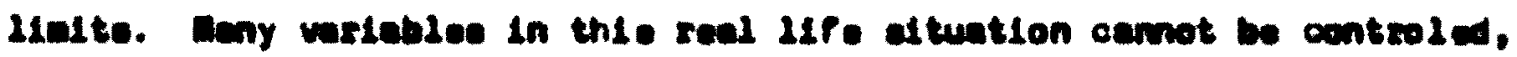
but wat goes on during sundey service on bo contrelled and thue of ve

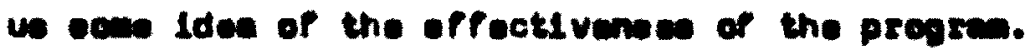

Since eociel peychology denle wore eith proup bohaviar then the other areve of paychology of ten the wothode ved do not land themeelves to thoht Ieboratory experimented ecntrol. Howover, If socited solentiste wis to oenduct experimente cutaide the inboratery, boginninge nuat be node w th the adoet aontzole that are not procluded by arel and practioal condiderations. The information gethored from an inteneive eduoationil experiment euch o the one prepeesd could be very uesful and precticel for poople Intereoted in real lifo control of religious attitudes. If an educatloral progres oen trul y mke pueple wore accepting of changes. It cen becone a vaoful teol when chense in a religloue institution beceses necosery.

Rallelowe authorlthe and church-oping people were beth very cooperative In the present etudy. If a elople experimont offere ece hape of preotical reculte it aght be poesble to Imeure furthor coserotian.

Finally, thle etudy ha provided a penel of eubjecte that alght prove uepul in conourlog the attitudes tewrd rollglow changes over tlaw. Since procent goorwe are aviliable on thle eovle, it would be poesible to readiniferer the scale in twalve wonthe and weoure chwoes over tine in the respondente' ettltudes. 


\section{Rerencuces}

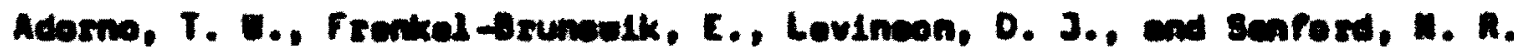

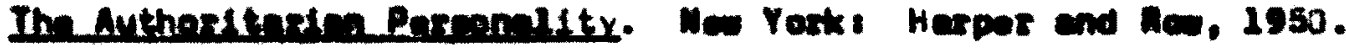

Allen, E. E. and HLtee, R. ". Fectore in rellgl aus ettitudes of

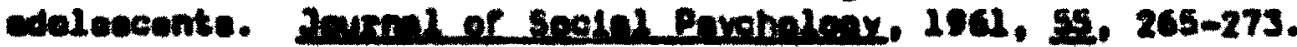

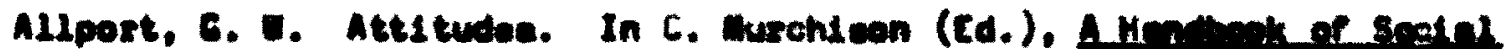

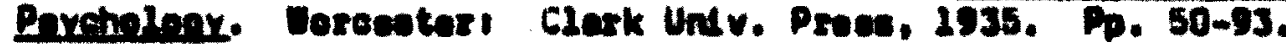

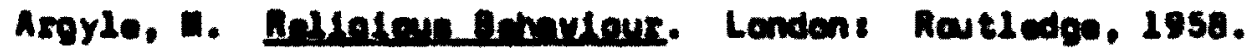

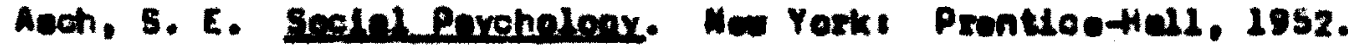

Bean, C. M. Trm poyohalegy of edhorence to the ald and of eoecptence of

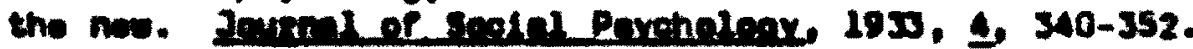

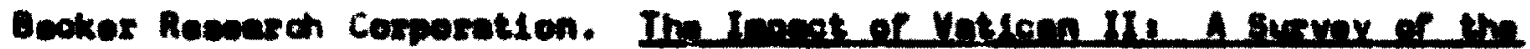

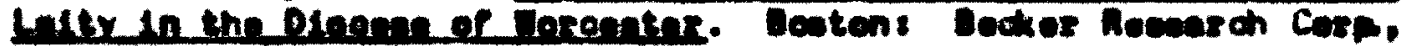
196.

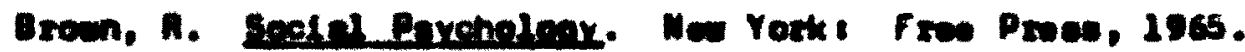

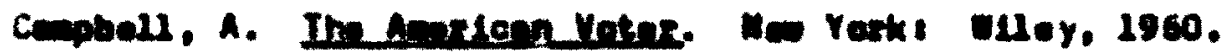

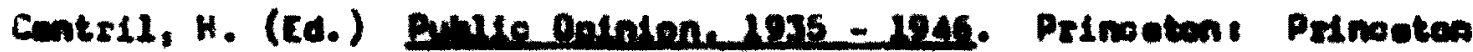
univ. Prens, 195.

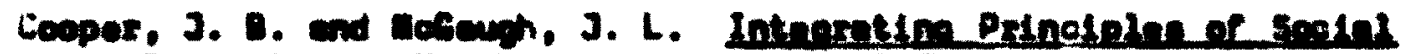

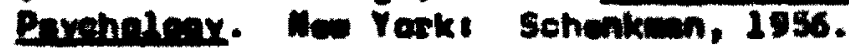

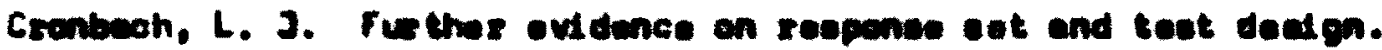

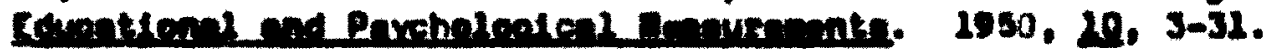

DLttev, J. E. Peyoholegy of Raligion. In G. Lindzey end C. Asenem

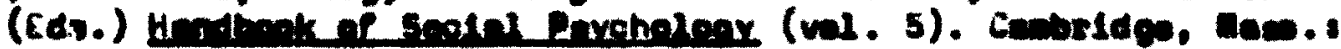
idsteen teoley. 1969.

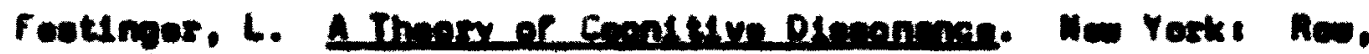
Potercen, 1887.

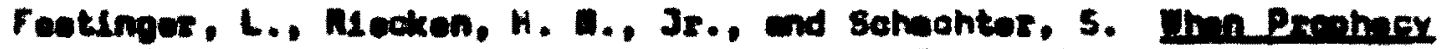

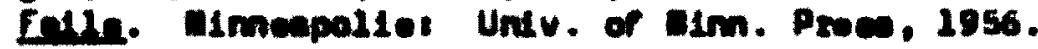

Flehter, J. H. The proflle of Cathollc rallolous 11fe. Anrtate Ieunal of seatelonx, 1952, 58, 145-149.

Godin, A. Eegormes for and rualotunce to the eclentiflo poyohalegy of

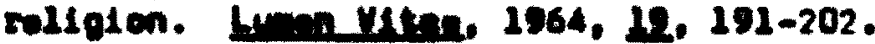




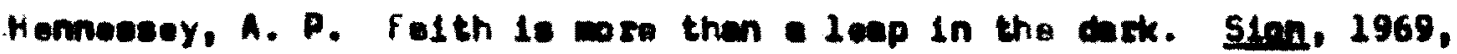
19. 6 .

kovlend, L. I., Jenie, I. L., and Kelley, H. H. Lomunlention and Pernungten. Londons oxpord Univ. Prese, 1953.

Katz, D. and Statiend, E. A prelleinary atetement to theory of ett1 tude otructure and chenge. In 5 . Koon (Ed.). Percholonks

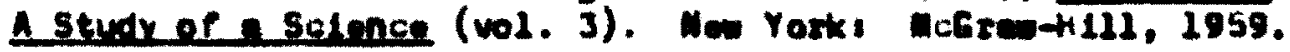

Kelley. H. H. Sel lance of membarohlp and realetence to chenge of group-enthored ett1 tudes. Himn Relintion, 1955, B, 275-289.

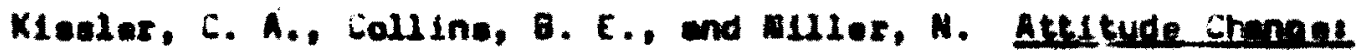
A Critlinl Andrele of Theoretsed Amreechen. New York: 110y. 1969.

Krech, D. and Crutohfield, R. S. Ihnery and Prohlera of Soint Puxcheloax. Mee Yorks Mchreeti11, 1948.

terpole, C. H. The oomparative auscoptibllity of three ege levele to the augoetion of group verwe expert opinion. Jeumel of secinl Paychology, 1933, A. 176-186.

Mencomb, T. A., Turnar, R. K., and Converse, P. E. Souid Pexcholeax. Wev Yorks halt, Rinohert, 1965.

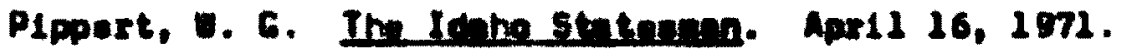

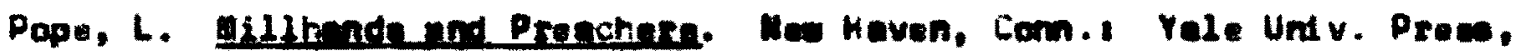
1942 .

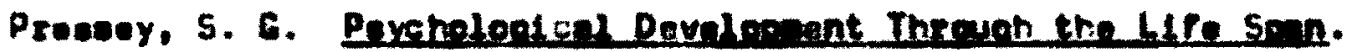
Hew York, Haxper and Rew, 1957.

Ryder. M. B. and weotoff. C.F. Uee of erel contreception in the Unitud States, 1965. 5ctench, 2966, 153, $1199-1205$.

Shoer. R. . and Centere, A. DLfrorences in attitudimel respenese under conditione of Inplloltiy eenipuleted group ealience. 3ryml of Perronality end sociel Prycholoax, 1970, 15, 125-132.

Stark. R. On the Incompatibllity of rellgion and eclances aurvey of American oreduate atudente. Jouml for the selentifie study of Antorion. $1963,3,3-20$.

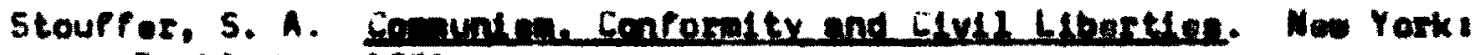
Doubladey, 1955 .

Thowe., 1. and Inent ock1, F. Int Polfth Penent in Euroes and Antige. Beotont Bedger, 1918-1920. 
51

Dalker, R. C. and Flzotto, A. The olezeyen to a variable in

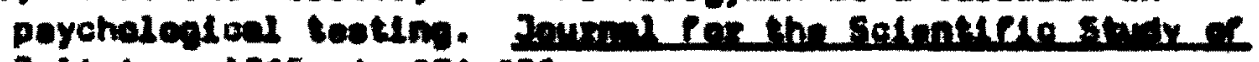
Aelinion. 4965, A. 234-236.

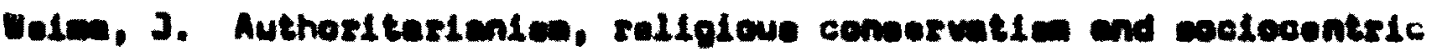

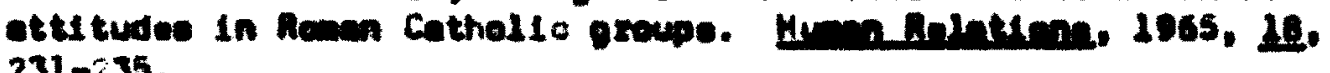
$231-235$.

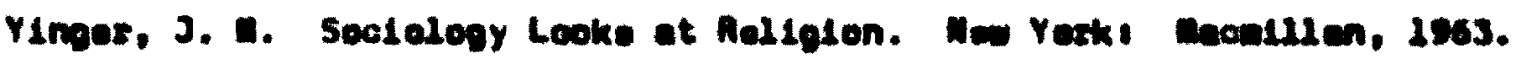




\section{Apprastx}

\section{ATtITUDE scale}

Thie questennalre Io dealoned to mbble you to exproes your opinion on sea mottera of Importanos in the Cathollo Church. Pleapo

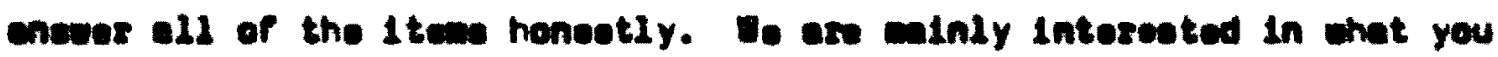
pereenily thick of these 1tewe. Pleape de not put your nawe on the

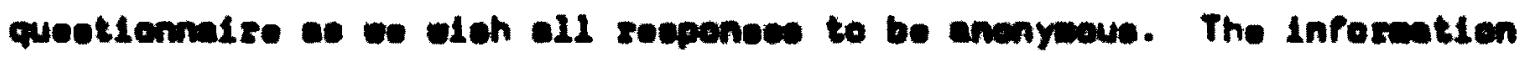

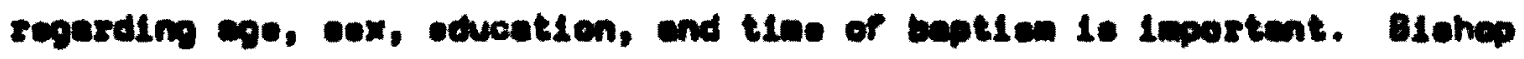

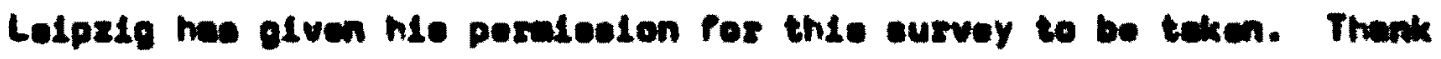
you. 
I. Hy ege at wy lest birthday wee yeare.

II. I ale.

I en Panale.

III. I wes baptized Into the Cathel10 Church a. an infent. as an edult.

IV. I have completed grade echool. high echool. ecene college. Pour yours of collego.

V. I attend wees regulerly (alnot every Sunday). irrequierly.

1. Wean and glrle ehould be allowed to be lectore and eerve naes.

1.

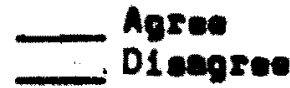

2. A married clergy could not render good enrvice in the Catholle Church.

2.

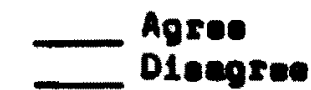

3. The Catholic Churoh 1o not the only true Church.

3.

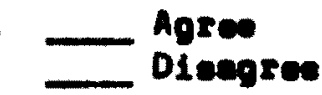

4. The blohop should nat confiru chlidren untll they are at leest entore in high eaheol.

4.

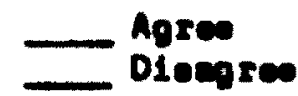

5. In genarel, and for the moet part, the chenges teking plece in the Catholic Church today are

5.

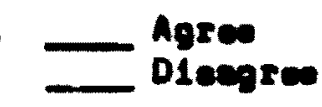
good.

6. Divorced-remerried Catholice chould be peralted to recolve the encremente (oepecially Comunion) even though theis eecond marriege lo invalid according to normel etandarde.

7. Saying the Roeary 10 not importent to being a good Catholite.

6.

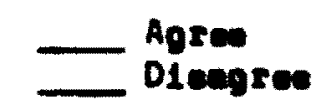

7.

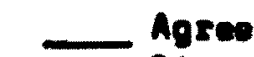

- Diegrea

8. It would not be a very good idee to lat priente and lay peoplo elect bichope.

8. Agrew DLeagres

9. Catholice ohould not be given the opportunity to fulfill thair Sunday obligation at Saturday oventing llase.

10. The Cathollo Church ahould not encourage wore joint wrohip arvioes ath nan-Catholice.
10.

9. Agres Diegrese

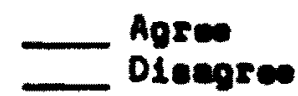


11. Prieste ahould not be allowed to merry.

12. To recedve Comunien otanding lnotead of

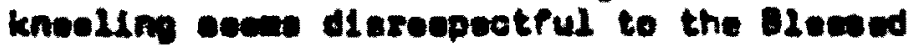
seorament.

13. Demen mut nover be allewed to bocene priteote.

14. ChLldren ohould not be ellewed to recelve thats firet Cemmunion althout poing to Confeesion.

15. Married clergy would render better eervicee then unerefled clorgy olnee thoy ould understand pasly probleno bettes.

16. To o good Catholic it is nacaeary to raprein prom eny uoe of artipledel bith contrel devisee and the blrth oontrol pLLI.

17. The Cathalle cleroy should preech ware Proquently on cociel probleme of our timos.

18. Evan chen the Pope opouke officlally on eny matter of falth or merrele, a Catholle cen et1ll Pallow hie wen conectenoa.

19. The Amorican Cothelle blohepe ehould atve aere offlald eupport to Cutholle boye the wh te teke the peoltion of eeneolentloue objector to ene.

20. Blehepe ohould elwaye be oheen from anong the wore eaneorvetlue ambere of the clergy.
11.

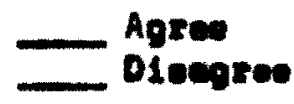

12.

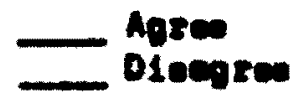

13.

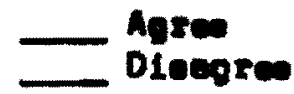

14.

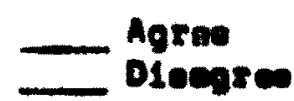

15.

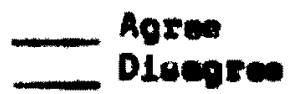

16.

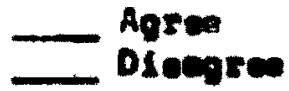

17.

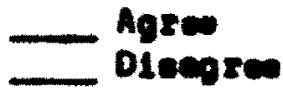

18.

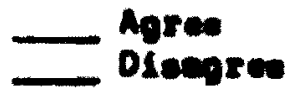

19.

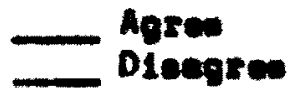

20.

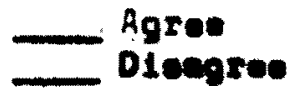

